\title{
Astrophotonics: astronomy and modern optics
}

\author{
S. Minardi ${ }^{1} \cdot$ R. J. Harris ${ }^{2,4}$ (D) $\cdot$ L. Labadie ${ }^{3}$
}

Received: 9 November 2018/Accepted: 2 June 2021 / Published online: 3 September 2021

(C) The Author(s) 2021

\begin{abstract}
Much of the progress in astronomy has been driven by instrumental developments, from the first telescopes to fiber fed spectrographs. In this review, we describe the field of astrophotonics, a combination of photonics and astronomical instrumentation that is gaining importance in the development of current and future instrumentation. We begin with the science cases that have been identified as possibly benefiting from astrophotonic devices. We then discuss devices, methods and developments in the field along with the advantages they provide. We conclude by describing possible future perspectives in the field and their influence on astronomy.
\end{abstract}

Keywords Astronomical instrumentation · Photonics · Astrophotonics

\section{Contents}

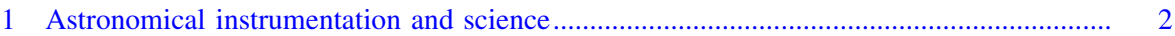

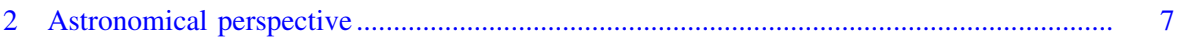

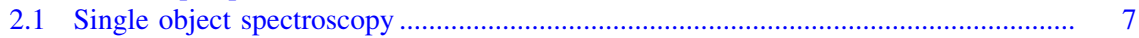

2.2 Integral field spectroscopy and multi-object spectroscopy: hyperspectral imaging ... 9

R. J. Harris

harris@mpia.de

L. Labadie

labadie@ph1.uni-koeln.de

$1 \quad$ AMS, Göschwitzer Str. 32, 07745 Jena, Germany

2 Zentrum für Astronomie der Universität Heidelberg, Landessternwarte Königstuhl, Königstuhl 12, 69117 Heidelberg, Germany

3 I. Physikalisches Institut der Universität zu Köln, Zülpicher Str. 77, 50937 Köln, Germany

4 Max-Planck-Institute for Astronomy, Königstuhl 17, 69117 Heidelberg, Germany 
2.3 High-angular resolution ................................................................................ 12

2.4 High-contrast techniques ............................................................................... 14

2.5 Metrology and calibration techniques................................................................. 15

3 A very short introduction to photonics ………......................................................... 16

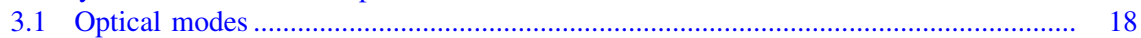

3.2 Modes and seeing ................................................................................. 19

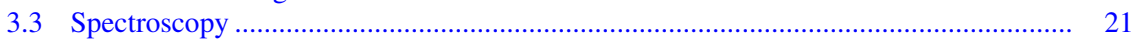

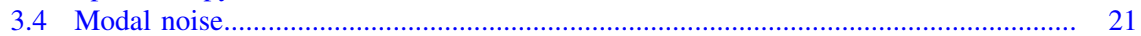

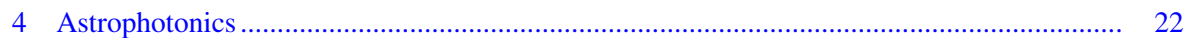

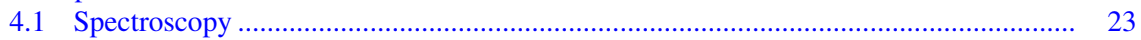

4.2 3D and multi-object spectrographs ..................................................................... 29

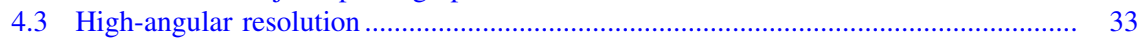

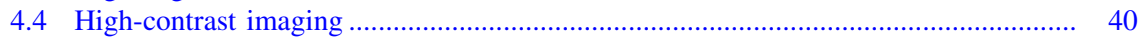

4.5 Metrology and calibration ................................................................................. 46

4.6 Detection enhancement through frequency conversion............................................. 53

5 Conclusion and future ............................................................................................. 56

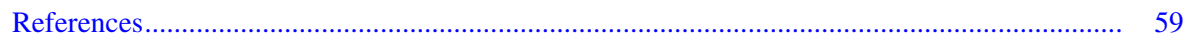

\section{Astronomical instrumentation and science}

Astronomy is without doubt the empirical science which more than others relies on the analysis of electromagnetic radiation, and as such its progress has often been correlated to the development of optical technology. The most obvious examples of the interaction between science and technology are the discoveries Galileo made at the beginning of the seventeenth century, by pointing his telescope, then the state of the art of optical instrumentation, at the sky. This simple act revolutionized our understanding of the universe, and these early astronomical observations drove the technological developments that allowed science to deliver telescopes of everincreasing quality. Before the end of the seventeenth century, Christiaan Huyguens was able to develop an aberration-corrected eyepiece allowing him to resolve the rings of Saturn, which were previously believed to be satellites, and detect the rotation of Mars. The invention of the reflecting telescope by Newton, which solved the problem of chromatic aberration, and the development of parabolic mirrors by Gregory $(\sim 1720)$, which solved the problem of spherical aberration, opened the possibility to build telescopes with apertures significantly larger than the refractive ones. By the end of the eighteenth century, William Herschel was observing the sky with reflecting telescopes exceeding half a meter in diameter (his largest telescope had an aperture of $122 \mathrm{~cm}$ ), enabling him to discover faint objects such as the satellites of Saturn and Uranus as well as to compile an extensive catalogue of 'nebulae'. The invention of the spectroscope by Fraunhofer (1814) and the subsequent discovery of the absorption lines in the spectrum of the Sun is another example of how progress in optical technology eventually changed the course of astronomy. The identification of the spectroscopic signature of chemical elements around 1850 gave astronomers a powerful tool to understand the structure of stars and of the universe, whose benefits we are still exploiting. A visual summary of the progression of optical technology and astronomical discoveries is illustrated in Fig. 1, which shows qualitatively how new technologies, similar to new paradigms, 


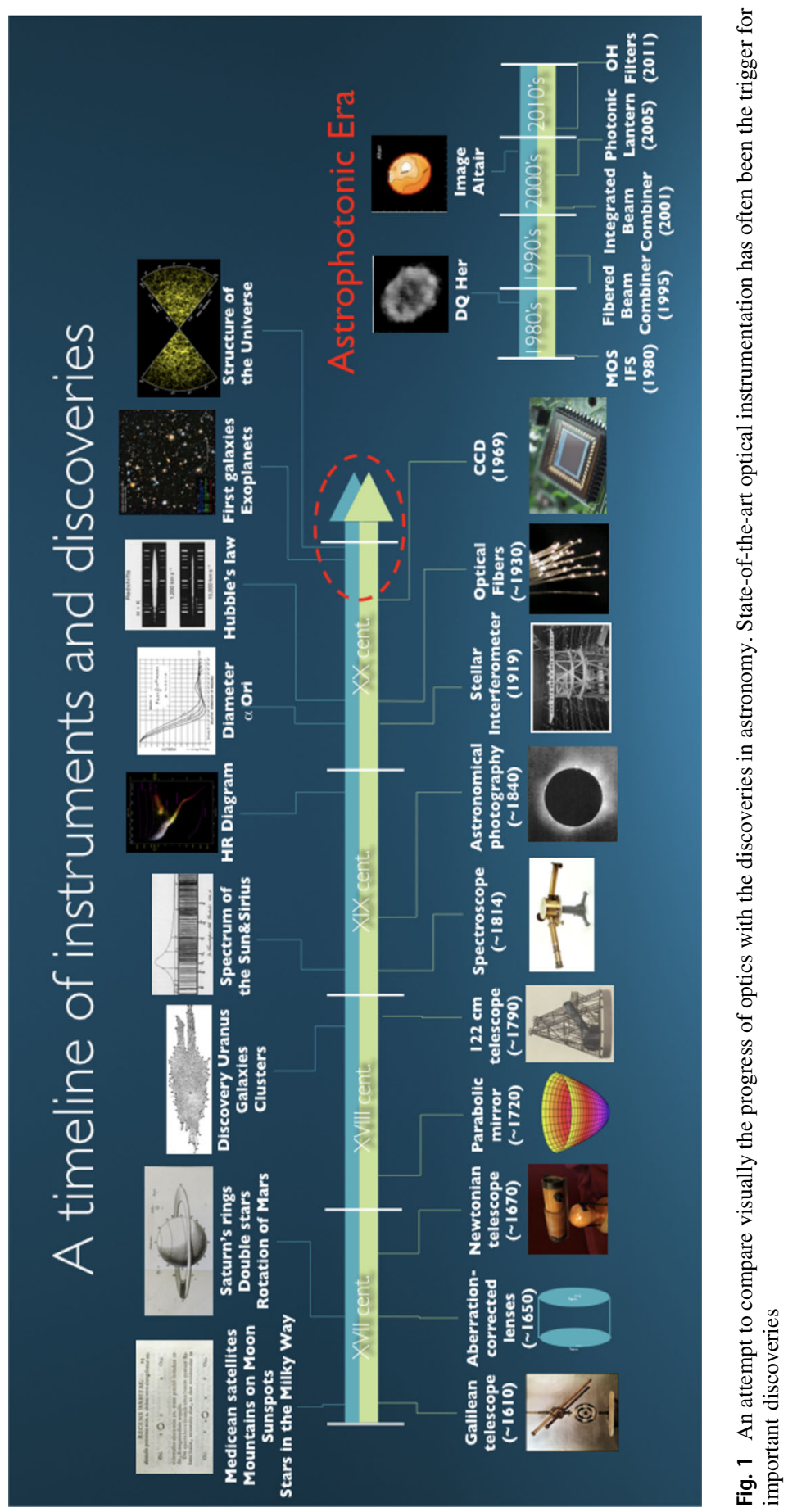


often require about a generation to bring their potential to fulfillment and open new avenues in astronomical discoveries (Kuhn 1962).

Today, the frontier in optics is represented by photonics, which is defined as the science dealing with the technologies for generating, transforming or using tailored light states to perform a task. As the word suggests, photonic devices control and manipulate photons in the way electronic devices do with electrons. Using this definition we can include devices such as micro-optics, laser sources, optical filters, optical fibers, and optoelectronic switches, which are commonly used in applications for telecommunications and digital data storage/readout. For this article, we will adopt a more restrictive definition of photonics and will deal mostly with elements requiring the wave approximation of optics to explain properly their operation. Given the impact of optical technologies in shaping the history of astronomy, it is not surprising that astronomers have taken an interest in photonic technologies and potential improvements they could bring to their instruments.

The first examples of what is called now 'astrophotonics' (i.e., the application of photonics to astronomical instrumentation Bland-Hawthorn and Kern 2009) date back to the late 1970 s when single optical fibers were used to connect telescope focal planes to spectrographs (Hubbard et al. 1979). The 1980s saw further developments, when optical fibers were used to improve the productivity of spectrographs (e.g., the Multi-Object Spectrograph (MOS) MEDUSA Hill et al. 1980) or to obtain hyperspectral images of extended objects (Integral Field Spectrograph (IFS) such as ARGUS Vanderriest 1980). Besides representing a major part of all existing astrophotonic instrumentation, multi-object and integralfield spectrographs have become essential tools of contemporary astronomy which enabled large scale surveys of the sky and kinematic studies of galaxies, both inconceivable only a few decades ago.

After the astonishing development of telecommunication photonic and these first successful demonstrations of astrophotonic applications, the 2000s saw a growing interest and expectation from the astronomical community towards photonics. Three potentially appealing characteristics of photonic over conventional 'bulk optic' instrumentation are (i) the potential reduction of size and mass of the instrument, (ii) the possibility to build highly multiplexed instruments, and (iii) the delivery of enhanced instrumental performance. The first advantage is clear in the case of integrated optics, i.e., a technology enabling optical devices manipulating light at spatial scales of the order of the wavelength. Complex free-space optics setups based on beam-splitters, folding mirrors, gratings and filters may be easily integrated in an optical fiber or a glass substrate of a few square centimeter area and few $100 \mu \mathrm{m}$ thickness. This makes the functional part of an instrument extremely compact and lightweight, a clear advantage especially for infrared instrumentation (the cryogenic enclosure is smaller and thus less demanding) and space missions. The miniaturization and the possibility to fabricate easily many identical copies of the integrated device makes astrophotonic instrumentation appealing for highly multiplexed instruments which enable a more efficient use of unique telescope facilities, which is a requirement for large-scale astronomical surveys. The classical example of this feature is provided by the introduction of optical fibers which made multi-object spectroscopy possible (see Sect. 2.2). Integrated optics could in the 
future allow for an even more aggressive multiplexing strategy leveraging on the inherently small size of the functional units of the instrument. Finally, astrophotonic devices could deliver instruments with unique performance, which could not be accomplished by other means. This is for instance the case of integrated optics beam combiners for stellar interferometry (Sect. 4.3) which deliver a highly precise visibility measurement through the use of single-mode optics, or the phase mask coronography, which can suppress efficiently light at the diffraction limit by means of micro-/nano-structured optical plates (Sect. 4.4).

Besides these advantages, there are intrinsic as well as contingent limitations in contemporary photonics which limit the widespread use of photonic solutions to astronomy. From the side of intrinsic limitations, we mention that the functionality of most of the existing photonic devices requires spatially coherent and relatively narrowband light sources, which is usually not the case of astronomical sources. As we will discuss throughout this review, the use of adaptive optics can solve the requirement for spatial coherence, while recent technological developments in dispersion control in integrated optics (e.g., photonic crystal fibers; Birks et al. 1997) may help extending the bandwidth of existing photonic devices. As for the contingent limitations, in the past 40 years integrated photonic applications have been driven mostly by the multi-billion-dollar-worth digital telecommunication industry. As is well known, digital communication protocols are relatively tolerant to noise and other spurious effects which degrade the signal-to-noise ratio (SNR). Digital signals can be retrieved even in the presence of relatively high noise level (e.g., SNR 3). Therefore, specifications of telecom devices on back-reflections or intensity/polarization contrasts are often in the 10-20 dB range, which are incompatible with the dynamic range requirements of astronomy, typically exceeding $40 \mathrm{~dB}$. Additionally, and in contrast to astronomy, light sources in telecommunication are coherent and therefore amenable to optical amplification processes with low noise overhead. This makes the losses of telecom devices a secondary issue for their use, so that devices with $5 \mathrm{~dB}$ insertion losses $(30 \%$ transmission) are common. Such losses are clearly not acceptable for a photon starved application such an astronomical observation. Moreover, photonic applications developed mostly the infrared spectral region, due to the existence in this spectral band of low-loss transmission bands in glasses and emission lines of laser materials. While in principle it is possible to reproduce the photonic functionalities in other optical bands, the high development costs require a medium to large market to justify research and development. The small size of the astronomical instrumentation market is usually insufficient to promote such a technological shift.

The adaptation of contemporary telecommunication technologies and overcoming the above-mentioned limitations represent the current challenges of the astrophotonic community, which will be discussed extensively in this article. Nonetheless, after decades mostly spent exploring these potentialities, astrophotonics is already starting to become a favorite companion of advanced astronomical instrumentation. For example, photonic technologies will be part of the instrumentation for Extremely Large Telescopes (ELTs) already from the very beginning, as for instance laser guide stars for adaptive optics (Ciliegi et al. 2018) and optical 
fibers as key components of multi-object and/or integral field spectrographs (Evans et al. 2016).

This review seeks to give a structured and comprehensive overview of the interplay between photonics, astronomy and instrumentation, highlighting in particular the current state-of-the-art in the field as well as the directions in which the community may look. A visualization of this approach is presented in the ringplot of Fig. 2, where astronomical instruments (middle ring) result from the, often contrasting, specifications dictated by the astrophysics of the object under study (inner ring) and the constraints of optical physics and technologies (outer ring). Instrumentation can therefore be discussed from the perspective of the astronomer and from the one of the optical/photonic engineer.

As opposed to other overviews of the field (e.g., Bland-Hawthorn and Kern 2009; Bland-Hawthorn and Leon-Saval 2017), this paper incorporates different branches of astrophotonics that are usually addressed separately, as for instance spectroscopy, interferometry/high-contrast imaging, and calibration of instrumentation. Additionally, we give an introduction to the physics underlying the function of photonic technologies, through an introductory section on optical modes and in the description of the instruments. Section 2 addresses, from an astrophysical perspective, specific science cases and related requirements that can directly benefit from the photonics approach. Consistent with the ring-plot, Sect. 3 introduces the optical physics perspective by summarizing the formalism of optical waveguiding, which is central to the understanding of photonic technologies. Section 4 presents the different families of astrophotonics devices from a performance-driven point-ofview in comparison to the theoretical background given in Sect. 3. Finally, Sect. 5 concludes our paper and summarizes future perspectives.

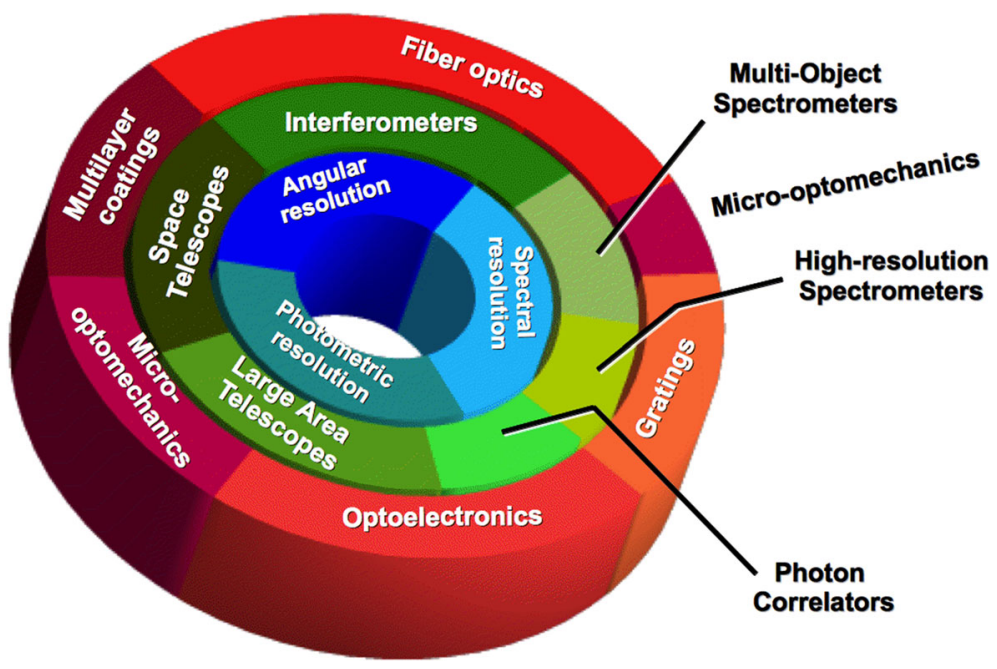

Fig. 2 Astronomical instruments (middle circle) develop in between the desired specifications derived from the astronomical target (inner circle) and the constraints imposed by the available optical technologies (outer circle) 


\section{Astronomical perspective}

Imaging and spectroscopic techniques - or the combination of them-are the two pillars supporting any advance in observational astrophysics. These two methods branch into several sub-techniques driven by specific science cases. The potential benefit of photonics-based solutions must be appreciated under the perspective of the scientific requirements associated with these two main techniques.

\subsection{Single object spectroscopy}

Spectroscopy is used in astronomy to investigate many properties of astrophysical objects, from chemical composition, to velocity structure, to the detection of exoplanets through the Doppler technique. This requires splitting the incoming light into its different wavelengths by means of dispersive optical elements (e.g., prism, grating).

As spectroscopy is exploited in the majority of the science cases in optical and infrared astronomy, we analyze hereafter the requirements of a subset of them.

Measurement of chemical compositions and effective temperatures: Low-resolution spectroscopy is usually employed to extract basic information on the source (such as the effective temperature, dominant stellar population or redshift) from faint objects, for which a large dispersion would lead to a spectral flux density well below the noise level. Resolutions with $R$ up to a few tens are used in deep galactic and extra-galactic surveys, and are usually realized by means of a set of interchangeable band-pass filters rather than spectrographs.

Solid-state species and broad spectral features such as ices (Pontoppidan et al. 2005), silicates (van den Ancker et al. 1999) or PAHs (Van Kerckhoven et al. 2002; Taha et al. 2018) can be studied at a resolution of a few hundreds to a few thousands in the visible and near-infrared regions, where the molecular bands are active (Véron-Cetty and Véron 2000; van Dishoeck 2004; Henning and Semenov 2013). Other uses of low spectral dispersion are slit-less and long-slit spectroscopy. In slitless spectroscopy, low resolution is necessary to avoid too many overlaps between the spectra of neighboring objects. Examples are found in space surveys such as GAIA, which measures the color of stars down to magnitude $G \sim 20$ with a slit-less spectrograph with $R \sim 20$. Long-slit spectrographs have been used mostly in the past to deliver spatially resolved spectra of faint extended objects such as galaxies or nebulae, but this technique has been mostly replaced by integral field spectroscopic methods, where photonic technologies play a key role (see below). Low-resolution spectra from Fourier-transform spectrographs are still common in mid- and farinfrared astronomy, where until recently only noisy, single-pixel detectors were available. The availability of new low-noise detector arrays in these bands has shifted instrumentation towards the simpler and more robust dispersive designs.

Measuring motion in the Universe: from stellar physics to exoplanets and (circum)stellar physics Besides investigating the chemical composition of astrophysical sources, spectroscopy is successfully and widely used for studying the radial velocities of celestial objects via the Doppler shift. Since the discovery of the 
first exoplanet around a Sun-like star using the radial velocity technique (Mayor and Queloz 1995) the number of exoplanets discovered using this technique has grown rapidly, including the discovery of a possible temperate terrestrial planet around the M dwarf Proxima Centauri (Anglada-Escudé et al. 2016). Taking advantage of the motion of the lines in the spectrum of an exoplanet with respect to those of the much brighter parent star, and to telluric contamination, high-resolution spectroscopy even permits to characterize the exoplanet atmosphere itself. This technique has for example been shown for $\tau$ Boötis b with CO lines using the CRIRES spectrograph at the Very Large Telescope (VLT) and might be implemented with the future METIS instrument on the ELT (Snellen et al. 2015). For these science cases, (very) high spectral resolution with $R \sim 100,000$ is typically required. These spectrographs exploit the échelle design where a grating is used at high diffraction orders, which are afterwards separated by a second dispersive element. To achieve a wavelength accuracy beyond the resolution limit, an extremely good stability of the instrument is required from the mechanical and thermal point-of-view as environmental disturbances may lead to drifts of the spectral lines being measured. Placing the optics in a vacuum vessel is highly advantageous but this requires a more demanding infrastructure depending on the size of the spectrograph. An essential tool for the observations is a stable wavelength calibration source to correct any drift of the optomechanical assembly of the spectrograph. Usually, gas cells/lamps filled with heavy ions are used as calibration units, a practice which is about to be complemented by the use of stabilized Fabry-Perot etalons and frequency combs (e.g. Steinmetz et al. 2008;) (see Sect. 4.5). Finally, the seeing-induced fluctuations of the barycenter of the light distribution at the slit are a major limiting factor to achieve the accuracy of wavelength measurements of stellar absorption lines. For this reason, techniques reducing the jitter on the spectrograph entrance are needed. Optical fibers are very efficient in doing that, but further measures to suppress modal noise need to be employed (so called modal scrambling techniques, see Sect. 4.1).

At intermediate spectral resolutions of a few thousands to tens of thousands, the gas kinematics in circumstellar environments (Goto et al. 2006; Pontoppidan et al. 2008; Gustafsson et al. 2008; Millour et al. 2011a) and galaxies can be spatially resolved using the techniques of spectro-astrometry (Takami et al. 2001) and spectro-interferometry. Motion of gas resulting from stellar winds and mass-loss is seen in high-resolution spectra where particular shapes such as P-Cygni profiles are observed. Importantly, the magnetism of stars can be investigated using highresolution spectro-polarimetry that reveals the splitting of atomic lines due to the Zeeman effect (Artigau et al. 2014).

Finally, for survey purposes accessing a large instantaneous field-of-view is a top-level requirement: the Radial Velocity Spectrograph (RVS) on GAIA accesses a field-of-view of $0.6 \mathrm{deg}^{2}$ at a spectral resolution of $R \sim 11,000$.

Solar system bodies There are numerous types of small bodies present in the solar system, mostly inhabiting the asteroid belt and Kuiper belt. Studies of these small objects within the solar system rely on detection and follow up capabilities, for which small telescopes are ideally suited, provided the objects are bright enough. 
Not only can these objects tell us about the chemical make-up of the solar system, they can also give us information as to how it formed.

In addition to the small bodies in the Kuiper belt and asteroid-belt comets can be used to inform theories about the formation of the solar system. To make useful discoveries high-resolution spectroscopy $(R>10,000)$ needs to be performed in the optical and near-infrared (Bailyn et al. 2007; Drew et al. 2010).

Finally, the atmospheres of solar system planets present an exciting opportunity, with further study allowing us to probe the composition and dynamics of the atmospheres to greater levels than previously achieved.

Low-mass brown dwarfs Brown dwarfs occupy the mass range between the deuterium and hydrogen burning limits, i.e., between the most massive planets and the smallest stars. The first brown dwarf was discovered in 1995, though their existence was hypothesized for many years before (Rebolo et al. 1995). Since the first discovery, the numbers and diversity in candidates have increased to hundreds, mostly due to surveys such as the Sloan Digital Sky Survey (SDSS) (York et al. 2000) and Two Micron All-Sky Survey (2MASS) (Gizis et al. 1999).

To increase the number of candidates will be the task of future photometric and spectroscopic surveys covering the optical and near-infrared (NIR). In addition, understanding the composition and processes in their atmospheres is particularly important, and spectroscopic surveys will be required to determine parameters such as composition, temperature and density. Due to the temperature of these objects low spectral resolution $(R<10,000)$ NIR spectroscopy is ideal.

Stellar variability There are many different causes of stellar variability, ranging from binary pairs, to stellar pulsations and to cataclysmic variables. Observations of these variable stars are extremely useful and they can be used to study many properties of the star, from determining the processes driving their variability, probing stellar evolution, fundamental properties and atmospheres. In addition, specific types of variable stars (e.g. RR Lyrae, Cephied variables) can be used to determine Galactic and cosmological distances (Freedman et al. 2001). In recent years the number of known members of these classes has grown thanks to gravitational lensing surveys (e.g., MACHO Alcock et al. 1993 and OGLE Udalski et al. 1994) and wide field surveys (e.g., ASAS Pojmanski 1997, ROTSE Akerlof et al. 2000 and HATNet Hartman et al. 2011).

Whilst time resolved photometry tells us much about these objects, there are also advantages to performing observations with time resolved spectroscopy, firstly to provide confirmation or clarification in the classification (Downes and Wallace 1996) and secondly to further probe the star. Most recently understanding variations in stars has been helping astronomers disentangle planetary radial velocity signals from those of effects such as sun-spots on the surface of stars (e.g., Oshagh et al. 2016; Perger et al. 2019).

\subsection{Integral field spectroscopy and multi-object spectroscopy: hyperspectral imaging}

Whilst spectrographs observing single objects are extremely powerful, they are limited in their capabilities to observe large numbers of objects. In an era where 


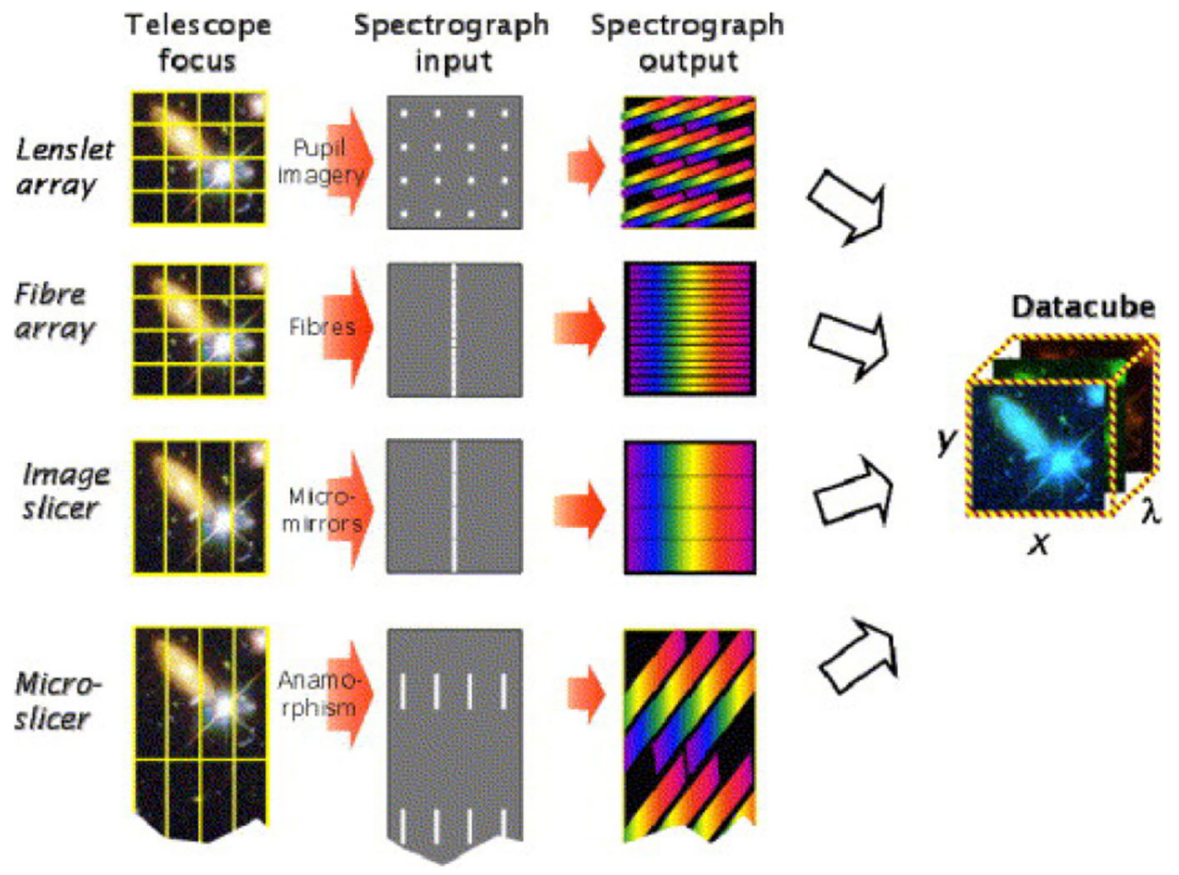

Fig. 3 Methods of integral field spectroscopy. The top image shows a lenslet array feeding the spectrograph, this is simple and allows for a large field of view, but requires the spectrograph to be close to the telescope. The second option, fibers, allows the spectrograph to be placed further from the telescope, usually at the cost of throughput. At the bottom, image slicers and microslicers generally allow high throughput, though also require the spectrograph to be close to the telescope. Image reproduced with permission from Allington-Smith (2006), copyright by Elsevier

many science cases require large statistical samples and with telescopes becoming fewer in number (due to their larger apertures costing more), more pressures are put on multiplexing, i.e., on the number of objects an instrument can observe at once. Spatial elements (spaxels) are fed from the focal plane into the spectrograph to produce a 3D data cube (see Fig. 3). There are two types of such instruments, which can be loosely classed into MOS and IFS. A MOS allows observations of multiple objects in the field of view (Ellis and Parry 1988) whist an IFS allows small patches of sky to be spatially resolved by an Integral Field Unit (IFU) (e.g., for observation of extended or spatially adjacent objects) (Allington-Smith 2006). To further increase the number and type of object that can be observed there is a combination of the two (using multiple IFUs) which is called Diverse Field Spectrograph (DFS) (Murray and Allington-Smith 2009). There are many science cases for all of the above and some are briefly outlined below.

In a 3D-spectroscopy instrument, the detector plays a central role in determining the final cost (Harris and Allington-Smith 2012), and it is therefore necessary to optimize the use of the detector pixels. Besides the spatial sampling at the telescope, it is necessary to consider the sampling element of the data cube (voxel; AllingtonSmith 2006), whose number equals the minimum required number of pixels of the 
detector. Hence, it is useful to introduce a figure of merit for the instrument, known as specific information density (SID, Allington-Smith 2006):

$$
\mathrm{SID}=\eta \frac{N_{r}}{N_{p}},
$$

where $\eta$ is the throughput of the instrument, $N_{r}$ is the number of resolution elements in the datacube and $N_{p}$ is the total number of pixels of the detector. The relation between the number of resolution elements and spaxels/voxels depends on the type of instrument and should be as close as possible to the maximum value of the SID to improve sensitivity. The SID is maximum when the number of pixels equals the number of voxels and the throughput is one. However, the number of resolution elements is in general smaller than the number of voxels because of oversampling; therefore, the maximum value for the SID will be smaller than 1. In a MOS, one target is sampled by a single spaxel and $N_{r}=N_{s} N_{\lambda} / f_{\lambda}, N_{\lambda}$ being the number of samples of the wavelength axis, $N_{s}$ the number of accessible targets, $f_{\lambda}$ being the oversampling factor. If we now assume that there are no unused pixels (condition to maximize SID) the number of voxels will be $N_{p}=N_{s} N_{\lambda}$. Taking $f_{\lambda}=2$ (Nyquist sampling of the wavelength), the maximum value for the SID will therefore be $1 / 2$. In an IFS, we will have in general $N_{s}=N_{x} N_{y}$ spaxels (a square array of spaxels is assumed) but the actual spatial resolution elements depend on the oversampling $f_{x}$ and $f_{y}$ of the telescope point spread function (PSF) along the coordinates $x$ and $y$. Thus the number of resolution element is given by $N_{r}=N_{s} N_{\lambda} / f_{x} f_{y} f_{\lambda}$. With Nyquist sampling in space and frequency, the maximum SID will be 1/8.

Galactic science To understand how individual galaxies form, what drives their evolution and what states they tend to, requires large data sets of spatially resolved galaxies. Therefore, large IFSs surveys are an extremely powerful tool. There are currently many large scale surveys such as MASSIVE (Mitchell Spectrograph), AMAZE (SINFONI) and KROSS (KMOS), providing data on hundreds of galaxies. These are being supplemented by even bigger ones (thousands of galaxies observed) such as MaNGA, SAMI, and Califa, plus soon the Local Volume Mapper (LVM). These surveys tend to provide direct information on two components, stars and gas.

New IFSs consisting of several hundred spaxels (e.g. MUSE at the Very Large Telescope (VLT)) are able to access resolved populations of stars in nearby galaxies or globular clusters (Bacon et al. 2010), making detailed kinematic and metallicity studies possible. For more distant galaxies, individual stars cannot be resolved but IFSs can still deliver the radial velocity distribution of their stars, a useful information to identify the morphology of galaxies (Croom et al. 2012).

Studies of the gas in galaxies are equally important. They can be used to trace the metallicity and abundances of the gas, telling us about the processes forming stars and the types of stars that will form and at what rate. In addition, gas can also trace the kinematics of the galaxy.

Combining information on both of these components in large data sets allows astronomers to answer the largest questions in galaxy formation and evolution today. These include how physical processes evolve with time, what regulates star 
formation, how metals build up, what drives gas inflows and outflows, the role of the local environment and what drives strong morphological transformations.

Cosmology Understanding the universe on the largest scales is one of the grandest aims of astronomy. How the universe expands, the composition of the universe and the physics governing everything are very large questions. To solve these, large sets of data are required. Obtaining these with single slits or fibers is difficult and time consuming. As such the trend is towards MOS instruments. For these surveys, the object is typically not spatially resolved and the science cases are mostly dominated by the desire to understand how galaxies and the corresponding luminosity functions evolve with time.

For this to be known, both the composition and the redshift of the galaxy must be understood. This requires moderate spectral resolving power instruments such as VIMOS ( $R$ of 200 to 2500), or AAOmega ( $R$ of 1000 to 8000). These instruments are used to perform surveys such as zCOSMOS, VVDS, VIPERS and GAMA. These instruments and surveys have led to many interesting discoveries, such as the combined discovery of periodic variations in the density of visible matter, baryon acoustic oscillations, by the $2 \mathrm{dF}$ instrument (Peacock et al. 2001). A current challenge is represented by the observation of faint galaxies with $z \simeq 2$ (the so-called cosmic noon, where star formation had its peak), for which the visible spectrum of their stars appears in the near-infrared. Because of chemoluminescence generated by the formation of $\mathrm{OH}$ radicals in the Earth's upper atmosphere, spectra of faint galaxies are difficult to acquire and evaluate. This problem has motivated the development of important astrophotonic devices which effectively suppress $\mathrm{OH}$ emission lines before a fiber-fed IR spectrograph (see Sect. 4.1).

Direct detection of exoplanets With photometric and time series observations of directly imaged exoplanets we can gain a wealth of information, constraining orbital parameters, size, temperature, surface properties and rotation rate (Traub and Oppenheimer 2010). To perform such observations is challenging, with the planet and star being separated by sub-arcsecond distances with a star-planet contrast of $10^{6}-10^{10}$. This means advanced Adaptive Optics (AO) systems are needed, often supplemented by a coronagraph to block light from the star. State-of-the-art systems often employ an additional spectrograph, some with IFU capabilities, such as the IFUs for SPHERE (Claudi et al. 2008), GPI IFU (Chilcote et al. 2012) and CHARIS (SCExAO) (Peters et al. 2012). IFUs not only allow the characterization of the planetary atmosphere (Bowler et al. 2010), but open up the possibility of further reducing contrast using techniques such as spectro-differential imaging (Racine et al. 1999) and molecule mapping (Hoeijmakers et al. 2018).

\subsection{High-angular resolution}

iImaging is the second main pillar of modern observational astrophysics, with the requirement of ever higher angular (or spatial) resolution (i.e., beyond the limit imposed by the seeing) being shared by a large number of science cases. 
In the last four decades, developments towards high-resolution imaging techniques have taken four main paths: development of large space telescopes, lucky imaging, ground-based adaptive optics, and optical long-base interferometry. The first three techniques allow the recovery, either partially or fully, of the diffraction-limit of the telescope, thus achieving angular resolutions ranging from some hundreds of mas to a few 10 mas depending on the wavelength band and the telescope aperture. Until recently, the main limit of space telescopes was represented by the limitations in weight and size of the payload of existing space carriers. This limited the aperture of the Hubble Space Telescope to $2.4 \mathrm{~m}$, but represented still (at the time of launch) a remarkable improvement in resolution ( $\sim 100$ mas in the visible) as compared to existing ground-based facilities. Lucky imaging techniques could in principle rival or even exceed the capabilities of HST, but entail a considerable data rate and post-processing overhead. Adaptive optics has seen an impressive development and widespread use in the last three decades, but (with some rare exception) is mostly confined to less demanding infrared bands and thus to resolutions above 100 mas. The European Extremely Large Telescope with its extreme AO system and an aperture of about 40 meters is expected to push this limit to a few 10 mas.

Currently, the only technique capable of extremely high resolution at optical frequencies is long-baseline interferometry, which allows the synthesis of diffraction-limited apertures of several 100 meters. Again the technique is mostly possible at near- to mid-infrared wavelengths, with resolutions ranging from 1 to 10 mas. These techniques have opened new opportunities in the investigation of the universe, the most significant achievements being related to the investigation of the early stages of stellar/planetary evolution and of the inner regions of galactic nuclei. Adaptive optics and speckle/lucky imaging in the infrared (Ghez et al. 1993; Leinert et al. 1993; McCabe et al. 2006; Correia et al. 2006) have complemented radial velocity surveys (Mathieu 1992) on young stars, confirming early studies showing that stellar multiplicity is a frequent property of solar-type stars with a binary rate larger than $\sim 50 \%$ (Duquennoy and Mayor 1991). The access to milliarcsecond resolution delivered by long baseline interferometry allowed furthermore to conclude that binarity was the norm rather than the exception (Sana et al. 2014).

High-resolution observations of circumstellar disks of nearby young stars with the Hubble Space Telescope (HST) (Padgett et al. 1999; Bally et al. 2000; Kalas et al. 2005, 2007), with AO-assisted ground-based instruments at Keck and VLT (Perrin et al. 2006; Duchêne et al. 2010; Garufi et al. 2017) or with long-baseline interferometers such IOTA, and VLTI (Monnier and Millan-Gabet 2002; van Boekel et al. 2004; Renard et al. 2010; Lazareff et al. 2017) resulted in a deeper knowledge of the first stages of planetary and stellar formation, significantly contributing to the improvement of theoretical models.

The possibility enabled by imaging long-baseline interferometry to observe the surface of giant stars (Buscher et al. 1990) stimulated a new development in the study of convection structures in evolved stars (Buscher et al. 1990; Paladini et al. 2018; Roettenbacher et al. 2016; Ohnaka et al. 2017) and for testing theoretical models of fast rotating stars (Monnier et al. 2007). 
Long baseline interferometry at Keck and VLTI elucidated the spatial properties of the circum-nuclear dust and the K-band nuclear emission in (AGNs) (Swain et al. 2003; Burtscher et al. 2016), while is the combination of milliarcsecond angular resolution and reverberation mapping may result in a new approach to cosmic distances estimates (Hönig et al. 2014).

High-angular resolution techniques have been successfully used to push the limit of astrometric precision in the micro-arcsecond range. Examples are some of the major results in the Galactic Center obtained thanks to the sharpest adaptive-optics images of the nuclear star cluster around Sagittarius A* (Schödel et al. 2002; Gillessen et al. 2017; Do et al. 2019) and the recently started 10- $\mu$ as campaign with the GRAVITY interferometer (GRAVITY Collaboration et al. 2017, 2020), which may further change our understanding of strong gravity physics, while building on previous technical progress achieved in the development of astrometric longbaseline interferometers such as PTI (Colavita et al. 1999), PRIMA (Delplancke et al. 2006; Sahlmann et al. 2013) and SIM (Unwin et al. 2008).

\subsection{High-contrast techniques}

High-contrast science may refer in the first place to the dynamic range of an image, or the amplitude between the readout noise and the saturation limit of the detector. This is particularly an important requirement for the study of stellar clusters, where a compromise between sensitivity and dynamical range needs to be found by adjusting the detector (low/high) gain mode. However, with the rapid expansion in the last decades of the field of exoplanets and planet formation, high-contrast science has developed strong ties to the detection and imaging of faint targets and structures in the immediate vicinity of a bright central source.

Spectacular results in the field of exoplanets and disks have been obtained with the use of coronagraphic instruments capable of significantly reducing the glow of the central star (Oppenheimer and Hinkley 2009). Giant planets have been imaged within $0.5^{\prime \prime}$ and arcseconds from the their parent A-type stars (e.g.Kalas et al. 2008; Marois et al. 2008; Lagrange et al. 2009) and offer hence excellent prospects for the future spectroscopic characterization of their atmospheres (Janson et al. 2010; Bonnefoy et al. 2016). Spatially resolved sub-structures such as warps and spiral arms have been observed in disks around AU Mic (Boccaletti et al. 2015) and Herbig stars (Benisty et al. 2015) using high-contrast techniques. Very highprecision interferometry in the near-infrared has been successfully employed to characterize the population of debris disks in the Solar neighborhood (Ertel et al. 2014). Beside the existing experience, nulling interferometry may experience a new revival, following the DARWIN/TPF studies (Fridlund 2004; Cockell et al. 2009), for the spectroscopic evidencing of biological biomarkers in the atmosphere of Earth-like planets (Kammerer and Quanz 2018). The recent discovery of a terrestrial, non-transiting, planet orbiting our closest neighbor Proxima Centauri (Anglada-Escudé et al. 2016) will certainly further motivate rapid advances in the field of high-contrast techniques.

Concerning the instrumental requirements applying to high-contrast techniques, the wavefront phase needs to be controlled as well as, or even more stringently than, 
for imaging or classical long-baseline interferometry. Since contrasts from $10^{-3}$ to $10^{-9}$ need to be achieved, effects related to imperfect Strehl ratios, control of pupil rotation, PSF centering, surface scattering, local intensity mismatches, differential polarization and long-term stability need to be addressed to achieve small innerworking angles (IWA) (Mawet et al. 2012) or deep and stable nulls (Mennesson et al. 2014a). For science cases relevant to exoplanets and planet formation, the requirement of observing in the thermal infrared ( $L$ to $N, Q$ astronomical bands) is of high relevance since here the flux contrast between the central star and the planet/ disk is more favorable than in the near-infrared or in the optical regimes. Considerations on the spectral richness of infrared spectra in terms of dust \& gas tracers and bio-signatures is a further motivation to operate at longer wavelengths. While a high total throughput is desirable to be sensitive to the faintest objects, the possibility to observe in broadband conditions is of high importance as well.

\subsection{Metrology and calibration techniques}

While not strictly related to one specific science case in astrophysics, the techniques employed for calibration and metrology in support of ground- or space-based observations are indispensible for an optimal interpretation of the science data.

One approach, definable as a passive calibration and likely the most used, consists in observing an astrophysical standard with exactly the same instrumental setup as the one adopted for the science target to identify features extrinsic to the object of interest and calibrate them out. The correction of strong telluric features in spectroscopy, the measurement of photometric standard stars for photometry, and the acquisition of so-called PSF reference stars for spatially resolved imaging are common examples of passive calibration.

Beside this, it is also common to use active calibration systems when a precise knowledge of the time-dependent long-term drifts and stability/uniformity is required, which may be difficult to monitor with on-sky calibration. A good example is high-resolution spectroscopy with a typical resolving power of $R \simeq 100,000$ when precise radial velocity measurements are sought for the detection of planets. Since the relative spectral shift of the stellar lines must be determined to a precision on the order of $\mathrm{m} / \mathrm{s}$, meaning a precision of $\sim 1 / 1000$ resolution element, high-stability calibration sources such as Thorium-Argon lamps or iodine cells have been employed to track instrumental drifts. While spectral lamps have been central for the operation of the HARPS optical spectrograph, the ESPRESSO spectrograph at the VLT will make use of spectral templates of superior stability and accuracy delivered by a laser frequency-comb Pepe et al. (2010). Artificial sources are also employed to assess the spatial flatness of a detector response, or the quality of the sky thermal background suppression at infrared wavelengths. In wide-field imaging applications, static and dynamic field distortion effects need to be traced, which is typically achieved using an artificial scene of widely and uniformly distributed point sources. Finally, the effective suppression of unwanted sky lines at optical and nearinfrared wavelengths by means of carefully tailored narrow-line filters is also highly desirable. 
Metrology becomes critical when unavoidable drifts and flexures of a large facility or instrument hamper the ultimate expected accuracy. Mechanical movements can be traced with high accuracy using interferometric methods with a stable, narrow line laser source. For instance, a Helium-Neon laser metrology can achieve (sub-)wavelength position accuracy at $633 \mathrm{~nm}$ with an intrinsic relative precision better than $2 \cdot 10^{-9}$. Some application cases can be mentioned: the laser metrology system of the high-precision astrometry instrument Gravity/VLTI launches its 1.9 $\mu \mathrm{m}$ laser beam from the interferometric lab up to the telescope pupil to trace back the path of the astrophysical beam (Lippa et al. 2016). Considering that the astrometric angle $\Delta \alpha=\Delta \mathrm{OPD} / B_{p}$, the uncertainty on the length of the projected baseline needs to be sufficiently small not to dominate the overall error budget. With a laser metrology, the precision on the length of the interferometric (projected) baseline $B_{p}$ greatly surpasses what is typically delivered by pointing models; another example of critical metrology need is found with the NEAT (now Theia) project of space-based high-precision astrometric mission (Malbet et al. 2012; Theia Collaboration et al. 2017). The small differential displacement of an Earth-hosting star with respect to the background reference stars due to the orbiting planet can only be measured if the uncertainties on the geometry of the focal plane array (FPA) are calibrated to sub-microarcsecond over many arcminutes (a precision below $10^{-5}$ pixels). An interferometric laser metrology system illuminating the FPA can help to retrieve the position of the PSF centroid (Crouzier et al. 2016).

The instrumental requirements inherent in active calibration and metrology are clearly driven by an excellent knowledge and understanding of the implemented hardware: this means that particular care needs to be taken to study the spectral content, stability and repeatability of a calibration lamp. Modern laser sources have made enormous progress in that respect. The characterization of the transparency range, optical quality and modal content of any passive component involved in the calibration chain (e.g. filters, optical fibers, phase modulators) must result from dedicated lab testing and, ideally, rely on high-TRL devices. Finally, the possibility to simplify the optical design of any calibration or metrology system for stability should be considered as an important requirement: in this sense, optical fibers that can efficiently transport, filter or mix light from different physical locations will play an increasingly important role in astronomical instrumentation.

\section{A very short introduction to photonics}

In this review, we present astrophotonic instrumentation not only according to their astronomical use, but also from the perspective of the employed photonic technologies. To this end, we use this chapter to introduce basic photonic concepts, such as waveguides and optical modes. These concepts will be used in Sect. 4 to classify astrophotonic instruments and discuss their properties.

Photonic components can be broadly divided into passive and active devices. Passive devices are optical elements introducing a static modification to the properties of light. In these devices, the light power is preserved or, more often, is reduced by losses. In contrast, active devices can dynamically modify the state of 
light by means of an interaction with an external agent. The output light power in active devices can be greater than its input, the gain being supplied by an external power source (e.g. electricity). As mentioned, in this review we will discuss photonic applications to astronomy based on components modifying the properties of light on spatial scales of the order of the wavelength of light. Under this classification, we can include micro-optics, phase masks such as gratings (see Sect. 4.1), or vortex phase masks (see Sect. 4.4) but not conventional lenses, which do not require the structuring of an optical surface at the micro-nano-scale.

A fundamental component falling in our classification of passive photonic devices is the optical waveguide. This is an heterogeneous optical medium consisting of a region of space with high aspect ratio (the core) characterized by a refractive index higher than its surroundings (the cladding) and transverse dimensions comparable to the wavelength of light. In such a medium, light can be confined in the core and propagate along the long axis (or longitudinal axis, conventionally oriented to coincide with the z-coordinate axis) thanks to the phenomenon of total internal reflection. The physical principle underlying waveguiding can be exemplified by simple considerations in the frame of the geometrical optics approximation. Neglecting light interference effects, we can show that light can propagate in the core of the waveguide if the external divergence angle of light (with respect to the longitudinal axis) is smaller than the numerical aperture of the waveguide:

$$
N A=\sqrt{n_{\mathrm{co}}^{2}-n_{\mathrm{cl}}^{2}}
$$

with $n_{\mathrm{co}}$ and $n_{\mathrm{cl}}$ being the refractive indices of the core and the cladding, respectively. The exact modeling of waveguides with transverse core dimensions comparable with the wavelength of light requires the solution of wave-equations and the introduction of the concept of spatial modes, as outlined below.

Optical waveguides can come in the form of a glass fiber or as an element of an integrated optical circuit, i.e., waveguides manufactured on a glass substrate. The state of light in waveguides can be manipulated by means of more complex passive components analogous to macroscopic devices, such as beam splitters (optical couplers), mirrors (Bragg gratings and micro-resonators) or phase plates (birefringent waveguides).

As active devices of interest for astrophotonics, we will consider mainly lasers and phase modulators. Lasers are optical media in which the population of electrons of a radiative electronic transition is inverted with respect to the state of thermal equilibrium. The population inversion is typically obtained by injecting power in the medium as optical radiation or electrical current. Light resonant to a radiative transition in an inverted medium is amplified coherently because of the occurrence of stimulated light emission. Lasing media are typically placed in an optical resonator, where light can be amplified by many orders of magnitude by passing repeatedly in the amplifier thanks to multiple reflection in the resonator. The laser resonator can be fabricated within a single optical fiber or waveguide using FBGs as mirrors, or manufactured in semiconductor waveguides within a laser diode. Photonic phase modulators are devices using an electrical signal to induce a local 
variation of the refractive index in a waveguide, which can advance or retard the phase of a guided optical field. They are typically based on the electro-optical effect, by which a constant electrical field can alter the birefringence properties of the medium. By including a phase modulator within an integrated Mach-Zehnder interferometer amplitude modulators can be realized as well.

\subsection{Optical modes}

An important concept in photonics is the optical mode, which is a stationary state (or eigenstate) of an optical system originating from its boundary conditions and the wave nature of light.

Mathematically, modes are solutions of differential equations which describe the physical system under study. Optical modes are solutions of the electromagnetic wave propagation equation:

$$
\nabla \times \nabla \times \mathbf{E}-\frac{n^{2}(\mathbf{x})}{c^{2}} \frac{\partial^{2} \mathbf{E}}{\partial t^{2}}=0,
$$

which is straightforwardly derived from the Maxwell equations. Here $n(\mathbf{x})$ is the spatially varying refractive index and $c$ is the speed of light. This equation is usually cast into an eigenvalue problem assuming a harmonic dependence in one or more coordinates. As an example, consider the case of the weakly guiding optical waveguide, i.e., a waveguide satisfying the relationship $\Delta n=n_{\mathrm{co}}-n_{\mathrm{cl}} \ll n_{\mathrm{cl}}$. In this case, we can safely assume that the electromagnetic field is transverse with respect to the axis of the waveguide and that the vectorial components of the electric field are decoupled. This allows writing the wave equation in a scalar form for the amplitude of one of the polarization directions of the field:

$$
\nabla^{2} E_{x, y}-\frac{n^{2}(\mathbf{x})}{c^{2}} \frac{\partial^{2} E_{x, y}}{\partial t^{2}}=0 .
$$

If the refractive index profile of the core is invariant along $z$, the $z$-(longitudinal) component of the electric field has a harmonic dependence on time and $z$ :

$$
E_{x, y}(x, y, z, t)=\psi(x, y) \cdot \exp [i(\beta z-\omega t)]+c . c . .
$$

As a consequence, the transverse profile of the field $\psi(x, y)$ obeys the following eigenvalue equation, derived by substituting Eq. (5) in the scalar wave equation Eq. (4):

$$
\left[\frac{\partial^{2}}{\partial x^{2}}+\frac{\partial^{2}}{\partial y^{2}}+n(x, y) \frac{\omega^{2}}{c^{2}}\right] \psi=\beta^{2} \psi,
$$

where $n(x, y)$ is now the refractive index distribution in the transverse $(x, y)$ plane, which attains its maximum in the region of the core. If the refractive index distribution has a peak, the eigenvalue equation has a discrete set of solutions, which represent the transverse modes of a waveguide. The eigenvalues are found by imposing everywhere continuity in value and derivative to the field $\psi(x, y)$, a 
consequence of $n(x, y)$ possessing at most a finite discontinuity in value (Snyder and Love 1983, Chapt. 33). Step index waveguides with circular cross section, i.e., waveguides with a cylindrical core of constant refractive index and abrupt transition to the cladding, are a particular subgroup of waveguides which possess mode profiles in analytical form. They represent a good approximation of real optical fibers, which can support one or more discrete modes, the modal behavior being parametrized by the normalized frequency $V$ :

$$
V=\frac{2 \pi}{\lambda} a \cdot N A,
$$

where $a$ is the core radius and $\lambda$ the free space wavelength of the light. For $V$ smaller than 2.405 , circular waveguides can support only a single mode. Asymptotically the number of modes supported by a step index waveguide with circular cross section is given by:

$$
N=\frac{V^{2}}{4}
$$

Modes can also have a longitudinal attribute, as is the case of optical resonators, regions of space where light is trapped such as in the volume between two parallel plane mirrors. The mirrors introduce a boundary condition for the electromagnetic field similar to that of a vibrating string (the optical field vanishes at the mirror surface) so that only a discrete set of longitudinal waves characterized by an integer number of half wavelengths are supported by the resonator.

Modes can be excited by matching an external optical field to the modal field distribution at the boundaries of the photonic device. Examples are the excitation of modes in a fiber by illuminating its tip with a beam having the same spatial distribution of the mode or illuminating the semi-reflecting mirror of an optical resonator with light tuned to the frequency of its stationary waves. Modes form a complete orthonormal base for stationary fields sustained by the photonic component and thus their complex amplitude $a_{\mathrm{j}}$ can be obtained by projecting the exciting field distribution $E_{\text {ext }}(x, y)$ onto the mode profile $\psi_{\mathrm{j}}(x, y)$ at the interface:

$$
a_{\mathrm{j}}=\sqrt{\frac{n_{c o}}{2}}\left(\frac{\epsilon_{0}}{\mu_{0}}\right)^{1 / 4} \frac{\int_{S} \psi_{\mathrm{j}}(x, y) E_{\mathrm{ext}}^{*} d A}{\left[\int_{S}\left|\psi_{\mathrm{j}}(x, y)\right|^{2} d A\right]^{1 / 2}},
$$

where $S$ represents the external surface of the modal volume. The normalization in this case is chosen so that the power carried by the $j$ th mode is given simply by the square modulus of $a_{\mathrm{j}}$.

\subsection{Modes and seeing}

Highly multi-mode fibers can be described safely in the frame of the geometrical optics approximation. This gives us the possibility to use the brightness theorem (Born and Wolf 1997), to describe the seeing limited PSF of a telescope in terms of modes. Moreover, as the brightness theorem is basically a formulation of the second 
principle of thermodynamics (McMahon 1975), we derive a useful lesson regarding mode transformation devices. The brightness theorem states that the power per unit area and solid angle (the brightness) of the image of a source of light formed by a passive optical system cannot exceed the brightness of the source itself. Since the brightness is related to the temperature of the source, it is clear that a violation of the brightness theorem could allow a perpetual motion machine to work. In a lossless passive optical system the collected power is preserved; thus the brightness theorem is equivalent to stating that the étendue of the source and the image are the same, the étendue $\mathcal{E}$ being defined as the product of the source area $A$ and its solid angle divergence $\Omega$ :

$$
\mathcal{E}=A \cdot \Omega
$$

We now consider an optical system consisting of a seeing limited telescope focusing light on a multi-mode optical fiber placed in the focal plane. For the image of starlight in the focal plane of a telescope of diameter $D_{\mathrm{T}}$ and focal ratio $F_{\sharp}$, the ètendue can be written as

$$
\mathcal{E}_{\text {tel }}=\pi\left(\theta D_{\mathrm{T}} F_{\sharp}\right)^{2} \pi\left(\frac{1}{2 F_{\sharp}}\right)^{2}=\frac{\pi^{2}}{4}\left(\theta D_{\mathrm{T}}\right)^{2} .
$$

Here the angle $\theta$ represents the seeing. On the other side, the etendue of light propagating in a step-index optical fiber can be written as the area of the core of radius $a$ by the square of the numerical aperture:

$$
\mathcal{E}_{\text {fib }}=\pi a^{2} \pi N A^{2}=\frac{V^{2}}{4} \lambda^{2},
$$

where we have used the definition of the normalized frequency $V$ of the waveguide Eq. (8). In this case, $\lambda^{2}$ can be interpreted as the etendue of a single optical mode. Because in lossless systems the etendue of light is a constant, we can write

$$
\frac{V^{2}}{4} \lambda^{2}=\frac{\pi^{2}}{4}\left(\theta D_{\mathrm{T}}\right)^{2} .
$$

Recalling that the seeing angle can be roughly defined as $\lambda / r_{0}, r_{0}$ being the Fried parameter (the correlation length of the atmospheric refractive index distribution; Fried 1966), we obtain

$$
\frac{V^{2}}{4}=\frac{\pi^{2}}{4}\left(\frac{D_{\mathrm{T}}}{r_{0}}\right)^{2},
$$

in which the expression $\frac{\pi^{2}}{4}\left(\frac{D_{\mathrm{T}}}{r_{0}}\right)^{2}$ can be interpreted as the 'modal content' of the point spread function of the telescope.

The conservation of brightness has further implications which add complexity for astronomical instrumentation. In particular, it shows the impossibility to have a passive, lossless device converting multimode light into a single mode (Welford and Winston 1982). If such a device were possible, the brightness of light confined in the 
single-mode output should necessarily increase because of the reduction of thel'etendue, thus violating the second principle of thermodynamics. This is the reason why multimode devices with single-mode behavior such as the photonic lantern Leon-Saval et al. (2005); Birks et al. (2015) distribute multi-mode light to an equivalent number of single-mode waveguides.

In active devices, brightness is not preserved, but the corresponding reduction of entropy is compensated by the necessity to extract information from the system. This is for instance the case of an adaptive optics system coupled to a single-mode fiber, which uses the information of the wavefront sensor to correct the wavefront and concentrates the power in a single mode of the fiber. The reduction in entropy associated with the correction of the aberrations is largely compensated by the entropy increase of the wavefront measurement operation, which requires the absorption of light on a detector.

\subsection{Spectroscopy}

In a simplified dispersive spectrograph, the light enters through a slit that selects a small region of the field, the light is collimated, a prism, grating or other dispersive element disperses the light, and a camera lens focuses the light onto a detection surface. One of the most important requirements for spectroscopy is the achievable spectral resolving power $R=\lambda / \Delta \lambda$, which is usually driven by a particular science case. It determines our ability to measure flux densities at two nearby wavelengths separated by $\Delta \lambda$. For grating spectroscopy, for example, the spectral resolving power is given by

$$
R=\frac{m \rho \lambda W}{\theta D_{\mathrm{T}}}
$$

where $m$ is the diffraction grating order, $\rho$ is the ruling density (usually given in lines per $\mathrm{mm}$ ), $\lambda$ is the operating wavelength, $W$ is the illuminated grating width, $\theta$ is the angular seeing and $D_{\mathrm{T}}$ is the telescope diameter.

This means that in systems where diffraction is dominant $\left(\theta D_{\mathrm{T}}=\lambda\right)$ the spectral resolving power is limited by the properties of the diffractive element. However, in non-diffraction limited cases $\left(\theta D_{\mathrm{T}}>\lambda\right), R$ will be constrained by the implemented dispersion element (e.g. grating, prism), the diameter of the telescope feeding it, and the slit size itself. This dependence is well established in classical instrumentation and further information can be found in spectroscopy textbooks (e.g., Schroeder 1987).

\subsection{Modal noise}

As mentioned in the previous sections, multi-mode fibers contain different modes depending on their properties. Depending on the incident electric field, wavelength, fiber stresses and strains different modes will be excited. With a constantly moving telescope and changing atmosphere, this translates to an ever changing output illumination pattern from the fiber. In an astronomical spectrograph, this 
phenomenon is known as modal noise and results in a variation of the measured position of the light-spot barycenter on the detector, resulting in a measured wavelength shift.

Whether this variation is important greatly depends on the science case, where wavelength precision is not required this is not a problem; however, for highly precise measurements, e.g. exoplanet detection, this can seriously influence the accuracy of results (Rawson et al. 1980). There are various ways of controlling this, for instance agitating the fiber (Baudrand and Walker 2001) and this works sufficiently for the current generation of spectrographs, which aim for around $1 \mathrm{~m} / \mathrm{s}$ precision and work in the visible with many modes. However, this problem is particularly pronounced as the number of the modes within the fiber is reduced, statistically increasing the relative uncertainty due to this movement. The next generation of instruments, which will aim for higher precision and those working in the infra-red will be particularly affected.

It must also be noted, that reducing the number of modes to one (not including polarization), eliminates this effect. However, it has been noted that polarization then has a greater effect Halverson et al. (2015).

\section{Astrophotonics}

In this section, we review photonic devices developed so far for astronomical instrumentation and classify them by astronomical technique and photonic function. As indicated in Fig. 4, we distinguish the six main astronomical techniques (namely spectroscopy, high angular resolution, hyperspectral imaging, high-contrast imaging and metrology/calibration, detection enhancement) whose scope and requirements

\begin{tabular}{|c|c|c|c|}
\cline { 2 - 4 } \multicolumn{1}{c|}{} & Single Mode & Mode Conversion & Multi Mode \\
\hline $\begin{array}{c}\text { Spectroscopy } \\
4.1\end{array}$ & Fibre for Spectrograph & $\begin{array}{c}\text { IPS } \\
\text { PIMMS } \\
\text { OH-filters }\end{array}$ & $\begin{array}{c}\text { MOS } \\
\text { Scramblers } \\
\text { Fibre for Spectrograph }\end{array}$ \\
\hline $\begin{array}{c}\text { Hyperspectral } \\
\text { Imaging } \\
4.2\end{array}$ & 10 Spectro-Interferometer & & IFU \\
\hline $\begin{array}{c}\text { High Angular } \\
\text { Resolution } \\
4.3\end{array}$ & IO Interferometer & Adaptive Optics & \\
\hline $\begin{array}{c}\text { High Contrast } \\
\text { Imaging } \\
4.4\end{array}$ & 10 Nulling Interferometer & Phase Mask Coronagraph & \\
\hline $\begin{array}{c}\text { Metrology \& } \\
\text { Calibration } \\
4.5\end{array}$ & CW Laser Guide Stars & & Astrocombs \\
\hline $\begin{array}{c}\text { Detection } \\
\text { Enhancement } \\
4.6\end{array}$ & & Fulsed LGS \\
\hline
\end{tabular}

Fig. 4 Astrophotonic instruments can be classified according to the scientific purpose (rows) and the modal behavior (columns) of their key photonic component 
have been discussed in Sect. 2. The photonic functionality distinguishes three categories depending on the modal content of the key photonic component of the instrument, i.e., single-mode, multi-mode and mode transformation devices. Under single-mode devices, we include continuous wave lasers operating at a single longitudinal mode, or devices based on single-mode optical waveguides. Devices such as laser guide stars and integrated optics beam combiners for interferometry are, therefore, found in this category. Multimode devices are pulsed laser sources, which emit a multitude of longitudinal modes, or devices based on multi-mode optical fibers. Laser frequency combs or 3D spectroscopy instruments (MOS or IFS) are typical representative of this category. In between the single-mode and the multimode categories, we can find mode transformation devices, which modify the modal distribution between input and output. These are for instance passive devices such as the photonic lantern and diffraction gratings (MM to $\mathrm{MM}$ ), phase masks (SM to SM) or active devices as for example deformable mirrors in an adaptive optical system (MM to SM). The table in Fig. 4 thus gives an overview of the astrophotonic instruments according to the dual classification described above.

In the following pages the sub-sections are devoted to the aforementioned astronomical techniques, while paragraphs distinguish the various photonic functionalities underlying the instruments.

\subsection{Spectroscopy}

Single-mode spectrographs The mismatch between the diffraction-limited resolution of the telescope $\lambda / D_{T}$ and the seeing $\theta$ reduces the spectral resolving power of the spectrograph, which can be restored only by making the grating (and hence instrument) larger or reducing the size of the slit (which can cause a loss in light unless techniques such as image slicing are used). The smallest spectrograph is then in general the one for which $\lambda / D_{T}=\theta$, the so-called diffraction-limited spectrograph. From the point-of-view of astronomical instrumentation, a truly diffractionlimited spectrograph could offer, besides size reduction, two further main advantages. Firstly, improved stability through the elimination of modal noise in fibers (see Sect. 3.4), and second, a further reduction of cost, thanks to either massproduced components for the photonic market or smaller conventional components.

Astrophotonics aims to create diffraction limited spectrographs by feeding the spectrograph with one or multiple (SMFs) acting as spatial filters (a single-mode spectrograph). Whilst the idea of using spectrographs fed by SMFs is not new to astronomy, coupling starlight to them is notoriously inefficient, due to atmospheric turbulence (Shaklan and Roddier 1988). A sophisticated AO system is therefore still mandatory to reduce coupling losses. Before truly single-mode (SM) coupling was considered, there were attempts using few-mode fibers (FMF) in 1998 (Ge et al. 1998), though with a light loss still unacceptable for astronomical spectroscopy. This restriction means most current spectrographs are fed using multi-mode (MM) fibers. In recent years, developments in extreme adaptive optics (ExAO) systems now routinely allow high Strehl ratios and hence increasingly efficient coupling into SMFs. This is leading to the first generation of SMF fed instrumentation, with 
examples of coupling tests on SCExAO (Jovanovic et al. 2017b) and full instruments in Minerva-red (Blake et al. 2015) and iLocater (Crepp et al. 2016).

Single-mode reformatters and spectrographs To overcome the requirement of using ExAO while preserving a high-throughput, single-mode spectrographs fed by a photonic lantern (PL) Leon-Saval et al. (2005); Birks et al. (2015) have been proposed (e.g. the Photonic Integrated Multi-Mode Spectrograph (PIMMS); BlandHawthorn et al. 2010). PLs are tapered optical fibers which adiabatically transform a MM optical fiber into a collection of SMFs. Light injected at the MM end of the device is distributed without losses in the output SMFs provided their number is at least equal to the number of modes supported in the MM end. A lossless transition to fewer fibers is in fact prohibited by the brightness theorem (see Sect. 3.2). PLs come in the form of fibers Leon-Saval et al. (2005) or integrated 3D waveguides Thomson et al. (2012); Spaleniak et al. (2013) and can provide single-mode functionality to multi-mode fiber (MMFs). This quality, originally exploited for the development of astronomical notch filters (see below), allows in principle efficient coupling from seeing-limited telescopes, while allowing light dispersion with a compact single-mode spectrograph. The PIMMS concept has two possible implementations, either the SMF ends are rearranged into a linear array acting as the pseudoslit of a spectrograph (also known as reformatting of light), or they are fed to integrated spectrographs (see next paragraph). Further elaborations of the PIMMS concept have been conceived or developed. The simplest is the photonic Tiger concept (named for the fiber fed Tiger IFU Courtes 1982). This means the spectra need to be dispersed such that none of the inputs overlap. Alternatives have been put forward, to use ultrafast laser inscription (ULI) to write a 3D integrated optical component reformatting the multi-core fiber (MCF) into a long slit. These are either separate waveguides (Thomson et al. 2012; Spaleniak et al. 2013) or the waveguides are joined together to form a long slit (MacLachlan et al. 2016a). The advantage of this method is similar to conventional slit techniques, maximizing use of the detector. In addition, fiber reformatters, taking the point spread function (PSF) from the telescope and reformatting into a slit have now been proposed and tested in the lab, showing high levels of throughput (Yerolatsitis et al. 2017). These are efficient, but in their current form limited to low mode counts due to the complexity of the devices.Throughput in low-mode fibers can be improved by improving the PSF of the telescope with a low order AO system (Harris et al. 2015). A preliminary numerical investigation of the trade-off between telescope beam quality and throughput is presented in Diab and Minardi (2018). Some of these devices have been tested on sky either with a spectrograph (e.g. the TIGER spectrograph Leon-Saval et al. 2012), or without (e.g. the Photonic dicer, Harris et al. 2015, and the hybrid reformatter, MacLachlan et al. 2016b).

Integrated photonic spectrographs As astronomical instruments are constantly growing in size, techniques allowing the reduction of their size and complexity are very popular. In telecommunications, this has been an aim for many years, resulting in the delivery of mature devices on centimeter size scales.

The idea of using these technologies in astronomy was suggested as early as the mid 1990s by Watson $(1995,1997)$ and was initially based upon Arrayed Waveguide Gratings (AWGs). The AWG works in a similar way to a conventional 


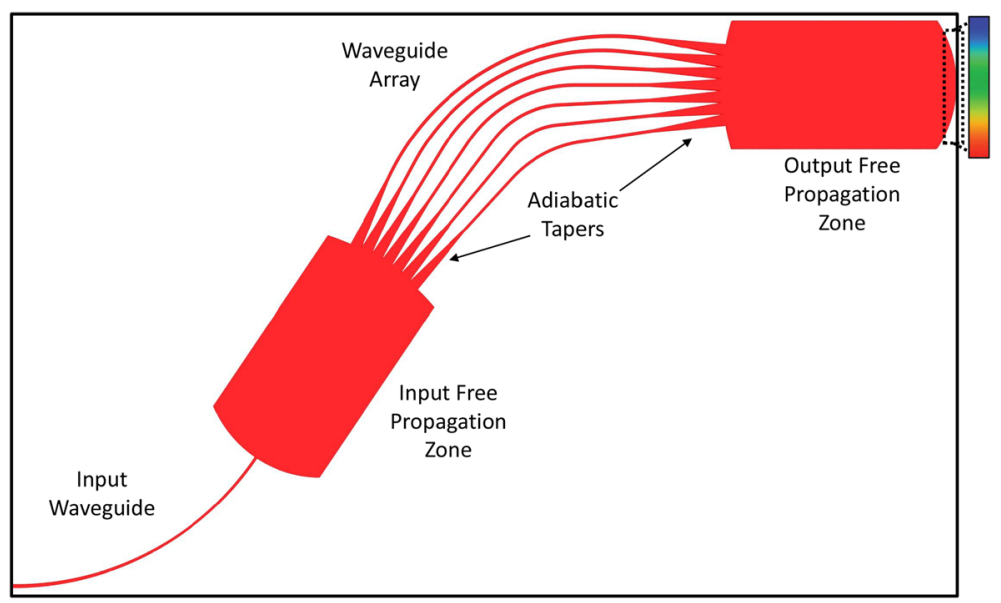

Fig. 5 A schematic of an astronomical Arrayed Waveguide Grating (AWG). Here the input is singlemode waveguide, which is fed by light from the telescope. This light passes through the input free propagation zone and enters the waveguide array where a phase difference is added (analogous to a grating). The light is recombined in the output free propagation zone and the spectrum is sampled either at the output of the chip or cross dispersed. Image reproduced with permission from Douglass et al. (2018), copyright by OSA

spectrograph, though embedded within a glass chip, which reduces size and alignment complexity. A sketch of the device is illustrated in Fig. 5. The initial single-mode waveguide can be considered as the slit, or feeding fiber to the spectrograph. The beam diffracts in the first free propagation region, which acts as a collimator thanks to the confocal curved surfaces of its edges (Goodman 2003, Chapt. 4). The expanded beam is sampled by an array of waveguides which, thanks to their curved paths, add a constant incremental phase difference to the light propagating in neighboring waveguides. The output of the array of waveguides is connected to a second free propagation region, this time acting as a camera lens. Similarly to a conventional spectrograph, spectral lines are focused at the curved exit surface of the free propagation region only if the optical path difference from the focus to any pair of outputs of the output array is an integer multiple of the wavelength. In telecommunication applications, single-mode fibers at the output of the second free propagation region are placed at regular intervals to collect the light of separate wavelength-demultiplexed communication channels. Since the incremental optical path difference introduced by the array of waveguides usually corresponds to several optical wavelengths, the AWG operates at high diffraction order $(\sim 25)$ like a conventional echelle grating. As a consequence, the free spectral range (FSR) of the AWG is very small, and their use for astronomy requires the introduction of a cross-disperser at its output to separate the diffraction orders.

At the time of Watson (1995) the AWG technology was not considered sufficiently developed to be used for astronomy. During the 2000s, the technology was reconsidered by Bland-Hawthorn and Horton (2006) who compared the AWG and Phased Echelle Grating (PEG) and concluded that the technology was now sufficiently developed for use in astronomy. 
The first on sky test of a modified commercial AWG was performed at the Australian Astronomical Telescope (AAT), formerly the Anglo-Australian Telescope in Australia (Cvetojevic et al. 2009). For this test the device was coupled to the telescope using a single, SMF. This led to low coupling efficiency with the seeing limited AAT, though high enough to pick up the atmospheric emission lines. As mentioned, due to the designed low FSR $(\approx 57 \mathrm{~nm})$ of the device, the IRIS 2 Tinney et al. (2004) spectrograph was used to cross disperse the output to obtain a useful FSR for astronomy. This first prototype displayed a spectral resolving power of around $R \approx 2100$ in the lab, which was suitable for low-resolution applications (see Sect. 2.1).

In the next set of experiments, various improvements were made (Cvetojevic et al. 2012). These included (1) optimizing production of the devices for efficiency, (2) removing the tapers of the input waveguides in the free propagation zone (FPZ) (which permitted an increased spectral resolving power), and (3) improving the coupling efficiency with the telescope using a PL. A single optimized AWG was then coupled to the IRIS2 spectrometer for cross dispersion.

Recently more efficient devices have focused on improving coupling, either through using a SMF with the extreme AO system SCExAO Jovanovic et al. (2015, 2017a) or with a PL input (Cvetojevic et al. 2017). However, the experiments using the PL were shown to induce modal noise due to a modal mismatch between the MMF and PL used (Cvetojevic et al. 2017). This shows the importance of correctly designing integrated photonic spectrograph (IPS) systems and photonic systems in general.

There have also been advances in developing custom AWGs for astronomy. These have been successfully manufactured for low spectral resolving power (e.g. Gatkine et al. 2016, 2017). Whilst a high spectral resolving power (of order $R=$ 60,000) AWG Stoll et al. (2017) has been designed, these are more difficult to manufacture and as of writing none have been successfully produced.

Another category of integrated spectrometers proposed for astrophotonic applications is based on the measurement of the temporal coherence of light in a waveguide. The so called Standing Wave Integrated Fourier-Transform Spectrometer (SWIFTS; Le Coarer et al. 2007) consists in a sensing waveguide coated with a regular array of gold nano-antennas, used to sample an optical standing wave trapped in the waveguide. To generate the standing wave, the outputs of a two-way waveguide splitter are connected to both ends of the sensing waveguide. Alternatively, light is injected at one end of the sensing waveguide, which is ended by a reflecting mirror. The standing wave is then formed near the mirror interface (Lippmann configuration). The nano-antennas are excited by the evanescent field of the guided mode and radiate light, which can be collected by an optical microscope. As a consequence, only a sub-Nyquist sampling of the standing wave is possible. This implies that the SWIFTS can be used at very high resolving powers $(100,000$ or more) on very small bandwidths. A possibility to achieve Nyquist sampling is to split the incoming light on an array of SWIFTS in Lippmann configuration, with the end mirror cut at an angle allowing to introduce an incremental phase shift for each SWIFTS, which can be used to densify the sampling of the standing wave. Even though they were initially proposed for 
astrophotonic applications Le Coarer et al. (2007); Kern et al. (2009) (see also Sect. 4.2), SWIFTS have intrinsically a low sensitivity, since the out-coupling efficiency of the nano-antennas should be low enough to avoid depleting the standing wave within a few optical cycles. Nonetheless their extremely small footprint and the possibility to paste the detector directly on the chip surface makes them attractive and sensitivity can be traded off for an increased multiplexing of the instruments.

$\mathrm{OH}$ suppression The constantly varying $\mathrm{OH}$ lines in the night sky are a large problem for ground based observations of faint objects in the NIR, as for instance galaxies of the 'cosmic noon' which possess a redshift of $z \sim 2$ (Bland-Hawthorn et al. 2004). To reduce the intensity of the telluric lines, the use of a tailored mask covering them in the focal plane of a high-resolution spectrograph has been attempted with a certain success (Iwamuro et al. 1994). However, focal plane masks cannot remove the Lorentzian tails of the PSF of the spectrograph, which scatter light in the interline spectrum and add background to the target spectrum. Though small, this effect is a significant contributor to noise in the spectra of the faintest objects.

To achieve ultimate suppression of $\mathrm{OH}$ lines, filtering should be introduced before the spectrograph. Following this approach, multi-notch filters Offer and Bland-Hawthorn (1998) and volume holograms Blais-Ouellette et al. (2004) were proposed, but the developments did not reach the level of prototype test on sky due to technical limitations of the fabrication methods. A more promising approach involves the use of FBGs in SMFs (Bland-Hawthorn et al. 2004, 2011). A FBG is a periodic refractive index variation within the core of a fiber. Because of the boundary conditions at the interface of media with different refractive index, the FBG acts as a periodic collection of semi-reflecting mirrors with tiny reflectivity. If the period of the grating is equal to an integer multiple of half wavelengths of the light, all these reflected waves can interfere constructively and the fiber acts as a narrow-band reflecting mirror (see Fig. 6a). As discussed, coupling starlight into an

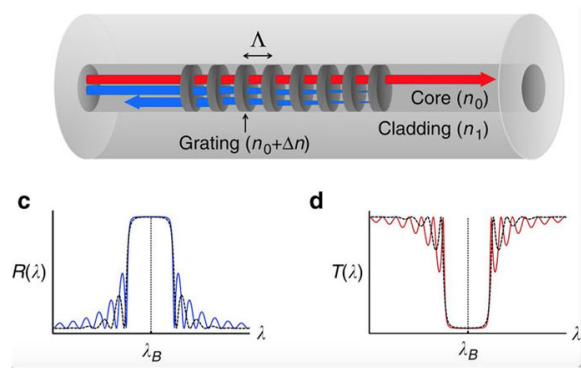

(a)

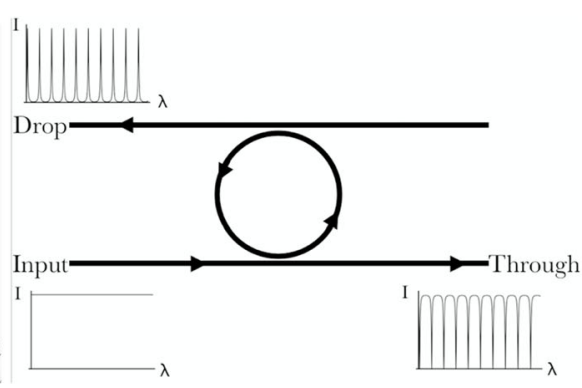

(b)

Fig. 6 a The top shows a schematic of a fiber Bragg gratings (FBG). Refractive index variations in the core of the fiber act as a Bragg reflector and reflect periodic wavelengths $\mathbf{c}$ back in the fiber whilst transmitting other wavelengths (d). Adapted from Bland-Hawthorn et al. (2011). b Schematic of a ring resonator, adapted from Ellis et al. (2017). Light from the 'input' waveguide is evanescently coupled into the ring and on resonance light is coupled to the 'drop' waveguide, off resonance passes back to the 'through' 
SMF is very inefficient, the reason why MMFs are preferred. The reflection line of a FBG written in a MMF splits however in several lines due to the slightly different effective wavelength of each guided mode which smear the reflection spectrum and reduce the overall rejection ratio of the filter. To get a single-mode-like FBG, MMF photonic lanterns were invented Leon-Saval et al. (2005). After conceptual development the devices were trialed on sky with the GNOSIS spectrograph. The AAT fed a 7 element IFU, one of which was picked off by a MMF. This fed a 1 x 19 core PL, turning the MMF into 19 SMFs. Each of these fibers contained a collection FBGs tuned to reflect $145 \mathrm{OH}$ lines in H-band; the filtered light was then fed to a reverse PL, turning the SMFs into a MMF which fed the IRIS 2 spectrograph (Trinh et al. 2013). Though this initial experiment was only used to examine the sky background and no objects were observed, they found that the $\mathrm{OH}$ suppression worked well.

Because of the time consuming nature of writing FBGs into SMFs, other methods of manufacturing them have been proposed, these include; (1) writing the gratings directly into a MCF Lindley et al. (2014), though currently this suffers from imperfections in the gratings due to focusing effects; and (2) using ULI to reformat the waveguides into a straight line, avoiding focusing effects and write the gratings there (Brown et al. 2012; Spaleniak et al. 2014).

The second generation instrument PRAXIS Horton et al. (2012); Ellis et al. (2016) achieved a strong suppression of $\mathrm{OH}$ suppression, however the expected removal of the scattered light background could not be proved, due to the high thermal background of the warm optics of the spectrograph (Ellis et al. 2020).

Performing $\mathrm{OH}$ suppression using a telecom add-drop filter based on multiple ring resonators has also been suggested (see Fig. 6b). These devices evanescently couple light from the input waveguide (the bus waveguide) into a ring. If an integer number of wavelengths matches the perimeter of the ring resonator (the resonance condition), light drops from the bus waveguide and is 'stored' in the resonator. A second waveguide placed opposite to the first one is eventually used to dump the light stored in the resonator. For light off-resonance, no stationary field can be sustained in the ring and light is completely transmitted through the first waveguide into the spectrograph (Ellis et al. 2011, 2017). To properly suppress the $\mathrm{OH}$ night sky emission, multiple rings are required and as such no on-sky demonstrators exist.

Incomplete scrambling and modal noise The majority of high-resolution, highstability spectrographs (with a few exceptions) are now fiber fed. This has two main advantages; the first is that as fibers confine the light, this allows the spectrograph to be optically independent from the telescope. This allows the final spectrograph to be placed remotely in a controlled environment, making stabilization easier. The second advantage is that fibers have better scrambling properties than other types of spectrographs (e.g., Heacox 1988). This means that as light travels along the fiber it is randomized, removing the imprint of the initial conditions of the light at the telescope. This helps with stability in the final spectrograph as it is less sensitive to atmospheric conditions, telescope movement etc. Despite being better, this scrambling is still imperfect and some residual imprint will be left at the spectrograph. To attempt to remove the residual imprint, two main techniques have been trialed. Fiber scramblers (Heacox 1986, 1988), use double lenses to scramble 
both the near and far field. Alternative fiber geometries have also been trialed, such as octagonal (Bouchy et al. 2013) or rectangular fibers (Jurgenson et al. 2016), the latter of which also acts as an image slicer to increase resolving power. Excellent results have been obtained also by a combination of a circular and octagonal fibers (Stürmer et al. 2014). Both techniques reduce or remove the problem of incomplete scrambling, particularly at lower spectral resolving powers, however at higher spectral resolving power another problem becomes apparent. Known as modal noise (see Sect. 3.4), this source of noise is due to the wave nature of light and vignetting in the spectrograph (Goodman and Rawson 1981; Lemke et al. 2011). To remove the modal noise in the final spectrum, the solution is to agitate the fiber (Baudrand and Walker 2001). This 'randomizes' the illumination pattern of the modes over time, evening out the resulting illumination pattern and reducing modal noise. The agitation solution is limited, however, in what it can achieve. There are many instruments under development, both at visible and NIR wavelengths with the science case of detecting earth like planets around a M-dwarf. With the longer wavelength of the light, or with smaller spatial sampling, removal of modal noise becomes more of a problem as the number of modes is reduced (Iuzzolino et al. 2014). Modal noise was such a large problem in the NIR GIANO spectrograph that the fiber section was replaced with a conventional slit in GIANO-B. It has been suggested that the PL could be used as an efficient mode scrambler (Birks et al. 2012). In particular, these investigations have focused on optimizing the scrambling properties of MCFs, using dissimilar core sizes (Haynes et al. 2014, 2018). Results have been promising, with significant improvements over conventional fibers (GrisSánchez et al. 2018).

\subsection{D and multi-object spectrographs}

These instruments are well established in astronomy, and can be roughly broken down into two types: MOS (multiple objects in different places) and IFS (the techniques is also known as hyperspectral imaging, spatially sampling a contiguous field containing one extended, or a few close objects). Early examples include slitless spectroscopy, which removes the slit from the spectrograph, allowing the objects to be dispersed using a prism onto a photographic plate or CCD (e.g., Comte and Surace 1994). This has the advantage that multiple objects can now be observed, but suffers from problems with crowded fields and extended objects due to overlapping spectra. This means the technique is still in use today, though is less common. Multi-slit spectroscopy can solve the problems of slitless spectroscopy, using a cut mask with many smaller slits (e.g., IRIS 2; Tinney et al. 2004; and VIMOS; Le Fèvre et al. 2003). This removes the unwanted stars, but it requires accurate manufacture. Re-configurable fiber optic systems now also exist, which increase versatility, such as placement in crowded fields and rearrangement of the fibers to stop resulting spectral overlap on the detector, increasing the efficiency with which the pixels are used (e.g., AAOmega; Sharp et al. 2006 and FMOS; Kimura et al. 2010). This technique also allows the light to be brought to a spectrograph on a stable platform further from the telescope. Whilst this introduces extra optics (such as the addition of a de-rotator at the Nasmyth focus), it means the 
instrument can remain fixed with respect to the gravity field, increasing stability (Bely 2003). Such instruments are now allowing huge surveys on smaller research grade telescopes (e.g., BOSS Smee et al. 2013). IFS first began in the 1980s (Vanderriest 1980), and it has rapidly expanded to become a mainstay within astronomy. It is achieved through four main methods: image slicers (e.g., GMOS; Dubbeldam et al. 2000; and NIFS; McGregor et al. 2003), lenslet arrays (e.g., SAURON Bacon et al. 2001), fiber slicers (e.g., Allington-Smith et al. 2002) and microslicers (e.g., Content et al. 2013), these are shown and described in Fig. 3. It must be noted that although these all use novel inputs, the spectrograph behind is very similar to the long slit analogue. DFS is also a rapidly expanding field, with instruments such as KMOS (Sharples et al. 2004) and FLAMES (Pasquini et al. 2002) paving the way for instruments planned for the next generation of Extremely Large Telescope (ELT)s (e.g., IRIS; Larkin et al. 2010). Image slicing can also be used to improve the spectral resolution of a spectrograph by reducing the width of the slit (e.g., Avila et al. 2012) and using the technologies developed for MOSs and IFSs allows the output from the telescope to be split into multiple replicated spectrographs (e.g., VIRUS; Hill et al. 2004).

Astrophotonic integral field units IFUs were first developed in the 1980s. They take a contiguous spatial sample of points and allow spectra to be taken. There are many types of conventional IFU (see Allington-Smith 2006), all with respective advantages and disadvantages.

Recently, multimode astrophotonic IFUs have been proposed in the form of hexabundles. This involves taking multiple MMFs, removing the buffer and reducing the the cladding through etching. These fibers are then arranged into an array and fused together to form a hexabundle IFU. By processing the fibers in this way, the fill fraction (the percentage of light sampled) can be increased. Care must be taken in the process not to cause cross coupling (Bryant et al. 2011), leading to a degradation in the signal.

To date, hexabundles are among the most successful spectroscopic components for astrophotonics. Following on from their initial development, they were used in the SAMI instrument on the AAT (Bryant et al. 2015), which was designed to survey the kinematic structure of galaxies. A future instrument HECTOR is currently planned (Lawrence et al. 2012), as an upgrade to SAMI it will use similar techniques, but with greater and also variable spatial sampling. This allows the cores of galaxies to be better sampled by the fibers.

IFUs consisting of a microlens array coupled to an array of single-mode fibers have also been proposed, to miniaturize astronomical spectrographs. As these are single mode, they would need to be fed by a diffraction limited beam to achieve a high throughput. This means they need either a small telescope, a long wavelength or extremely good AO correction. Currently there are variations in the form of the RHEA (Feger et al. 2014), a MCF fed spectrograph with a microlens glued on-top. Recently using MCFs with 3D printed microlenses on-top used as IFUs to feed diffraction limited spectrographs has also been suggested (Dietrich et al. 2017) and trialed on sky (Haffert et al. 2020).

Large scale IFUs for MOS has also been proposed for large telescopes with PSFs far from the diffraction limit. These would take the PSF from the telescope using 


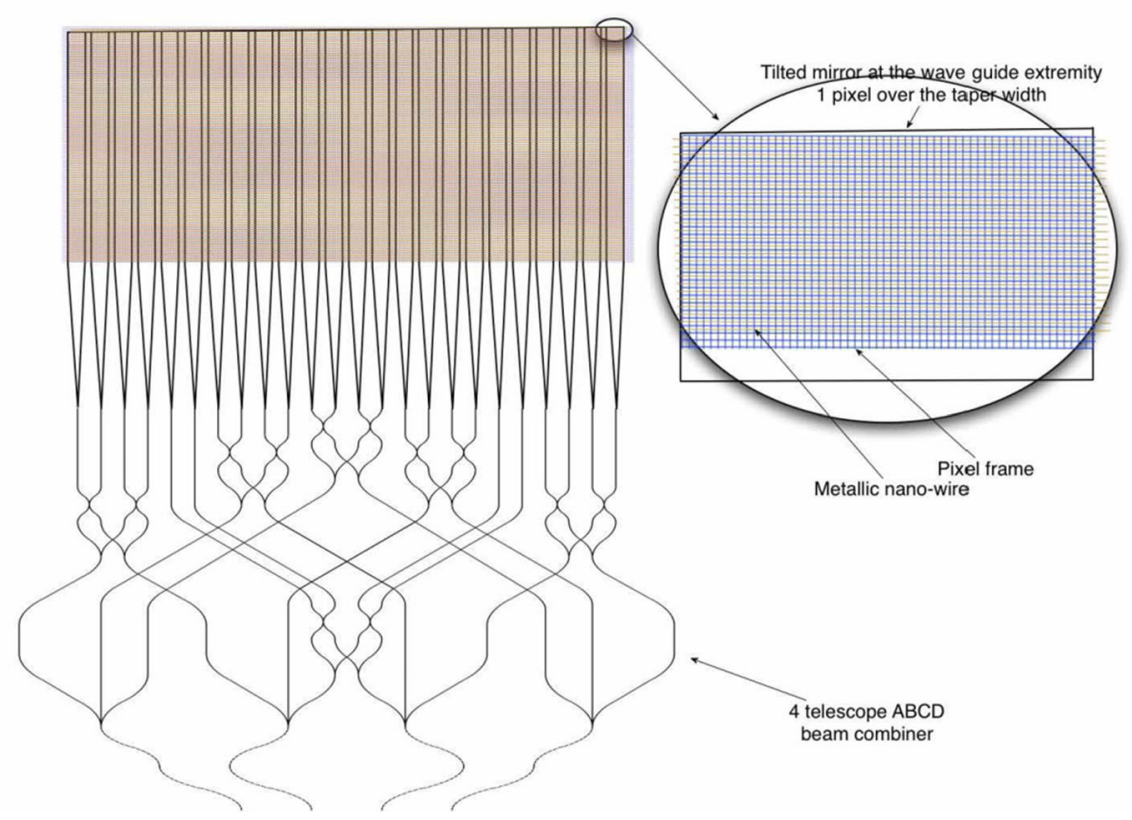

Fig. 7 Conceptual scheme of a fully integrated spectro-interferometer combining four telescopes. SWIFTS devices are printed upon the output waveguides of an integrated ABCD beam combiner, which can be read by detectors pasted on the surface of the photonic chip. Image reproduced with permission from Kern et al. (2009), copyright by OSA

highly MM PLs and convert them to few-mode (FM) PLs (Leon-Saval et al. 2017). Whilst the number of fibers would be large, converting down would allow access to SM technologies such as FBGs or ring resonators.

Photonic spectro-interferometry Spectro-interferometry is the high-angular resolution analogue of 3D spectroscopy, chromatically dispersing interference fringes to measure the variation of their visibility across the spectrum. If a sufficient number of different telescopes are combined, it is possible to retrieve interferometric images of the target for each wavelength (Millour et al. 2011b), thus creating a high-angular resolution (at the mas level) data cube as usually obtained at lower resolution with $3 \mathrm{D}$ spectroscopy. In astrophotonic interferometric instruments such as PIONIER or GRAVITY, the dispersion of the fringes is accomplished by interfacing the outputs of the IO component to an imaging spectrograph. Concepts and first experiments to accomplish the interferometric beam combination and spectral dispersion in one single chip have been put forward in recent years. Kern et al. (2009) proposed to combine an ABCD integrated beam combiner with a SWIFTS (see Sect. 4.1) to accomplish high-resolution spectro-interferometry (Fig. 7). The design consisted in splitting each output of the beam combiner in two straight waveguides terminated by a tilted mirror coating. The standing wave originating at the mirror surface can be retrieved by dotting the waveguide surface with a periodic array of gold nano-wires, which are excited by the evanescent field of the standing waves and couple the waveguide power to the free space. A camera 
can be pasted then to the chip so that the pixels correspond to the nano-wires, thus avoiding the use of relay optics, to collect the radiated light. To be resolved, the nanowires should be placed at distances much greater than the wavelength of the exciting light, thus realizing a sub-Nyquist sampling of the evanescent wave. However, the tilt angle of the mirror coated edge of the chip allows to shift spatially one standing wave with respect to the other, realizing de facto a sub-nanowireperiod sampling of the standing wave.

In the same article, a generalization of the concept to a planar device combining 8 input channels in spectro-interferometric mode was also proposed. Differently to the above mentioned scheme the sampling of the interference fringes would require phase modulation of the input channels or perhaps the use of redundancy in the fringe sampling to compensate for the large sampling period. None of these devices have been fabricated so far. A possible intrinsic limitation of these schemes is that the sampling of the standing wave should not deplete it significantly, thus only a small fraction of the optical power carried by the interfering waves will be effectively detected. On the other side, the complete spectro-interferometric device would be very compact so that the low sensitivity could be balanced by small dimensions and low weight.

The evanescent sampling of the field in the waveguide is also at the heart of a spectro-interferometric device integrating an array of waveguides with a diffraction grating manufactured and characterized very recently (Martin et al. 2017). This device exploits the possibility to couple in and out light in a waveguide by means of a suitable diffraction grating. Several parallel single-mode waveguides buried in glass are arranged in a non-redundant array. On top of each waveguide, a diffraction grating formed by an array of nano-void structures extract and disperse the signal carried by each waveguide. Suitable focusing optics are then used to combine in free space the spectra emitted by each waveguide in a multi-axial scheme. Both waveguides and the grating were manufactured by means of ultrafast laser inscription in Gallium Lanthanum Sulfide glass and were tested at a wavelength of approximately $1560 \mathrm{~nm}$. The grating was designed to give a resolving power of $R=13800$ but achieved a measured resolving power of $R=2500$, allegedly due to perturbations of the array periodicity and aberrations in the collection optics. This device accomplishes the wavefront filtering through coupling in single-mode waveguides and saves the space of the dispersive elements by means of a free-space multi-axial beam combination scheme.

Finally, a fully integrated, active spectro-interferometer was presented by $\mathrm{Su}$ et al. (2017) for wide-field aperture synthesis purposes. The planar integrated circuit delivers the light collected in a few on-axis as well as off-axis points of microlenses to 3-channel wavelength demultiplexers (centered at $1540 \mathrm{~nm}, 1560 \mathrm{~nm}$ and 1580 $\mathrm{nm}$ ); the outputs are then are combined pairwise (on-axis with on-axis input, offaxis with corresponding off-axis input) for each wavelength channel by $2 \times 2$ couplers. Heaters were mounted on the waveguides before the couplers to perform a fringe scan. Visibility functions of centered and laterally displaced slits were measured successfully in the laboratory. The prototype featured propagation losses of several decibels, making the device not yet suitable for astronomical use. However, the device is compact and can be in principle easily scaled up to combine 
several triplets of telescopes or sub-apertures at low cost, again features which could balance the low efficiency in a cost/benefit analysis.

\subsection{High-angular resolution}

Long-baseline interferometry The idea of using photonic components for long baseline stellar interferometry dates back to the beginning of the 1980s when a first analysis of the impact of optical fibers for the interferometric connection of telescopes was discussed (Froehly 1981). Fibers appeared as an economically viable alternative to free-space optical links, especially in the case of very long baseline $(>100 \mathrm{~m})$ interferometers. Short afterwards proposals for a fibered space interferometer were put forward (Connes et al. 1985) and research addressed the performance of optical fiber links respect to the requirements of ground- and space-based stellar interferometry (Shaklan and Roddier 1987, 1988). Despite the high insertion losses of single-mode fibers connected to a seeing-limited telescope, the absence of speckle noise and modal dispersion were soon recognized as the key features which could enable high-precision interferometry (Connes et al. 1987; Rohloff and Leinert 1991). To improve throughput, a method based on M-line spectroscopy (i.e., the technique allowing the angular separation of light carried by each mode of a multi-mode waveguide) was proposed to combine mode-wise telescopes connected by multi-mode fibers (Shaklan et al. 1992). Although an experimental proof of principle of the scheme was carried out successfully in the laboratory, further research focused only on single-mode fiber links. In particular, a scalable laboratory simulator of a fiber-linked astronomical interferometer was realized and tested (Reynaud et al. 1992). The demonstrator utilized fiber stretchers for fast adjustment of the differential optical path between telescopes induced by temperature changes and mechanical vibrations. The progress on laboratory tests as well as the first test on sky of a fibered beam combiner (see below) motivated the proposal of the OHANA interferometer, aiming at connecting with fibers the large telescopes on the top of Mauna Kea (Mariotti et al. 1996; Perrin et al. 2000). The prototype of a stabilized kilometric fiber interferometer was tested in the laboratory and an assessment of the impact of differential chromatic dispersion effects was undertaken (Vergnole et al. 2005; Kotani et al. 2005). Finally, first fringes in K band of 107 Her were measured connecting the two Keck telescopes with fluoride fibers but without active length stabilization (Perrin et al. 2006). Subsequent experiments with $20 \mathrm{~cm}$ telescopes connected with a pair of $300 \mathrm{~m}$-long silica fibers showed the possibility to obtain fringes on bright stars in $\mathrm{J}$ and $\mathrm{H}$ band, but pointed out the need for an active compensation system for the acoustic noise picked up by the fibers (Woillez et al. 2017). More recently (Lehmann et al. 2019), an actively stabilized $200+200 \mathrm{~m}$ fiber interferometer operating at telecom wavelength (1550 $\mathrm{nm}$ ) was laid over the $160 \mathrm{~m}$-long path outdoors between one of the CHARA telescopes and the beam-combination facility on Mt. Wilson. Fibers were protected from excessive thermal and mechanical perturbation by synthetic foam tubes. Stabilization over several minutes was possible despite the relatively short stroke of the fiber stretcher used to compensate the OPD $(150 \mu \mathrm{m})$. An overall r.m.s. noise level of $4 \mathrm{~nm}$ (corresponding to $\lambda / 450$ for $\mathrm{H}$-band) was achieved. The test was done 
in the frame of the ALOHA project, aiming at extending through photonic solutions the wavelength coverage of the CHARA array to the mid-infrared (MIR) (see also Sect. 4.6).

To date, fibers have not been used for connecting telescopes beyond the reported experiments. In contrast, the research on fiber links motivated the development of single-mode photonic beam combiners, which led to the realization of astronomical instruments, which are currently used for science. The first beam combiner exploiting photonic technologies was FLUOR, a K-band instrument based of fluoride glass fiber couplers (i.e., the fiber equivalent of a conventional beam splitter), which could combine two telescopes and measure the interference fringes as well as the light coupled in the fibers by each telescope (photometric signal) (Coudé du Foresto and Ridgway 1992). The instrument proved definitely the benefit of using the optical fiber as an effective spatial filter in the measurement of the fringe visibility with a precision of $1 \%$ or better (Coudé du Foresto et al. 1997b). FLUOR was initially tested at the auxiliary telescopes of the Kitt Peak solar observatory and moved (in an upgraded form) to the IOTA facility in 1995 (Coudé du Foresto et al. 1997a), where it delivered scientifically relevant data on star diameters. An improved version of FLUOR was used as commissioning instrument in K-band of the VLTI (Kervella et al. 2000) and is currently available at the CHARA array for high-precision visibility measurements (Coudé du Foresto et al. 2003). The drawbacks of filtering the spatially incoherent focal spot of a seeinglimited telescope with a single-mode fiber are a small average coupling efficiency (see Sect. 3) and a dramatic variation of the transmitted photon flux over time scales of the order of the coherence time of the atmosphere (Shaklan et al. 1992). While the low coupling efficiency limits sensitivity, the fast flux variability negatively impacts the signal to noise ratio of individual fringe measurements, requiring a postselection of the acquired frames (Tatulli and Duvert 2007). The combined use of an AO system and a fringe tracker (i.e., a device compensating in real time for the phase fluctuation induced by atmospheric turbulence) can solve both problems, as we have seen with the introduction of the second generation instrument GRAVITY at the Very Large Telescope Interferometer in Chile. To date, single-mode optical fibers are routinely used for the spatial filtering of the starlight in beam combiners which use multi-axial free space combination schemes such as AMBER (Petrov et al. 2007) and MIRC (Monnier et al. 2004).

The success of the FLUOR instrument motivated research of beam combiners based on integrated optics (Kern et al. 1997). Besides the potential for miniaturization of complex optical setups, the main advantage of integrated over fiber optics is the inherent thermomechanical stability of the optical paths in the former, due to the fact that waveguides are shorter and are rigidly attached to a solid substrate. The first 2-telescope planar integrated optics combiners for $\mathrm{H}$ band were manufactured with silica etching (component LETI) and ion exchange (component LEMO) in the late 1990s (Berger et al. 1999) and later tested on sky at the IOTA interferometer (Berger et al. 2001). The combiners had an area of a fraction of $\mathrm{cm}^{2}$ and delivered excellent visibility precision and accuracy at the $1 \%$ level on stars up to magnitude $(H=2)$. After this first demonstration of the potential of integrated optics, the 


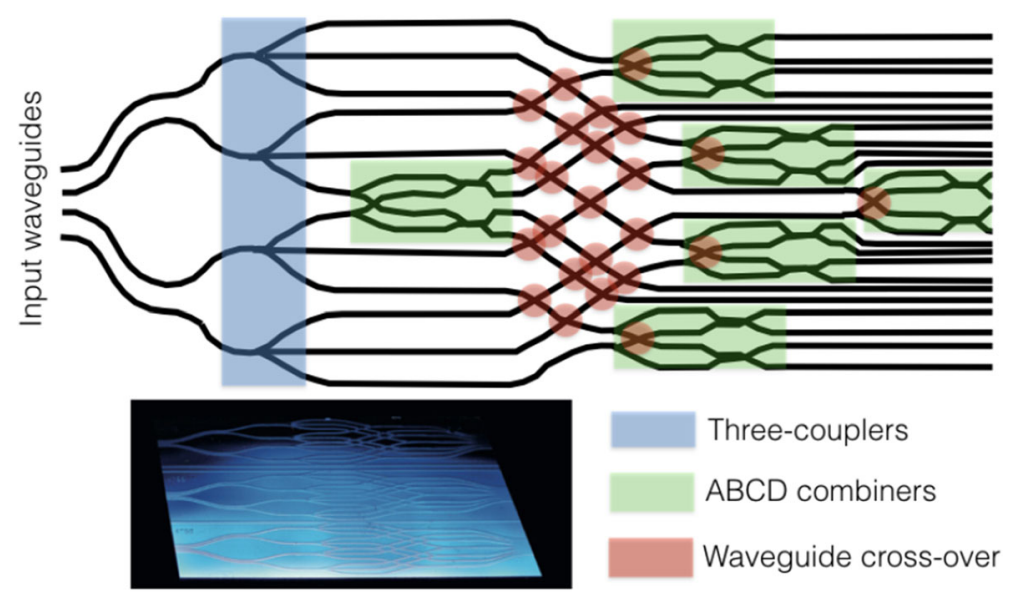

Fig. 8 Scheme of the integrated optics beam combiner of the PIONIER instrument, which can simultaneously retrieve the mutual coherence function of starlight collected by 4 independent telescopes (adapted from Benisty et al. 2009). The light from the four telescopes is coupled to the 4 input waveguides of the chip. Four three-couplers (highlighted in pale blue) divide the input light across 12 channels, which are mixed pairwise by the ABCD units (highlighted in green). The four outputs of each $\mathrm{ABCD}$ unit deliver the 4 quadratures of one of the 6 possible pairs of input fields. Because of the planar geometry of the integrated optics chip, the combination of each possible pair of inputs is achieved only by crossing the waveguides at large angles (the so called cross-overs, marked with the red dot) which could introduce some cross-talk between the interferometric channels. Bottom on the right a photograph of a chip with three identical chips (Credit: IPAG Grenoble)

challenge became to scale up the integrated components to combine a larger number of telescopes (Berger et al. 2000), a functionality which would be rather impractical to implement with fiber coupler technology. The IOTA facility was again used as a testbed for the first 3-telescope integrated beam combiner (Berger et al. 2003), which enabled initial interferometric imaging capabilities thanks to the measurement of the closure phase. Developments of the near-infrared combiner technology allowed to deliver by 2009 a first laboratory demonstrator of an H-band 4-telescope combiner (Benisty et al. 2009) (Fig. 8), which was afterwards used in the visitor instrument PIONIER at the VLTI (Bouquin et al. 2011) and delivered excellent interferometric images of close binary stars and protoplanetary disks. Today, an improved component operating in $\mathrm{K}$ band is at the heart of the GRAVITY instrument (GRAVITY Collaboration et al. 2017), an AO-assisted instrument capable of combining simultaneously the four 8-meter telescopes at VLTI and reaching a limiting magnitude of $(K=17)$ in fringe tracking mode.

Ongoing research on photonic beam combiners can be divided into three categories: (1) exploration of new combination geometries, (2) increase of the number of combined telescopes, and (3) extension to wavelengths longer than $2.4 \mu \mathrm{m}$ (absorption band of silica). In the first category, we mention the attempt to harness the three-dimensional (3D) structuring capabilities of ULI (Gattass et al. 2006) in various glasses. One strategy is to manufacture traditional pairwise integrated beam combiners (based on a cascade of couplers) exploiting the third dimension to avoid waveguide cross-overs, which introduce detrimental cross-talk 


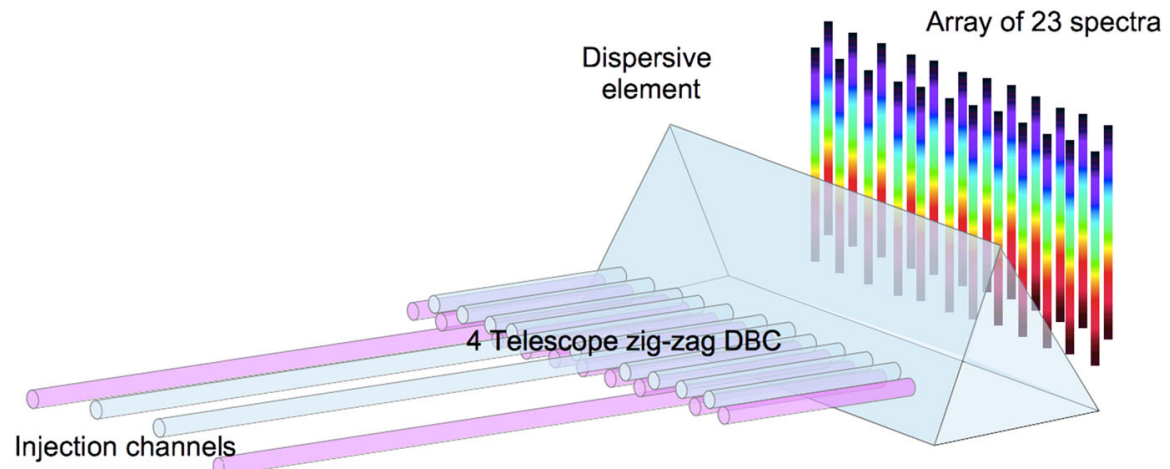

Fig. 9 Three dimensional photonics can significantly simplify the design of interferometric beam combiners. The scheme of a 4-telescope discrete beam combiner with zig-zag geometry (Diener et al. 2017), used in spectro-interferometric mode, is illustrated in the figure. Light is injected in 4 waveguides connected to an array of 23 evanescently coupled waveguides where interferometric beam combination occurs. The waveguides are disposed on two layers mutually shifted by half a period to ease the dispersion of the light in the vertical direction. In the figure, the waveguides of the two layers are differently colored, to help the reading. Collimation and focusing optics of the spectrograph are not included in the figure for simplicity

between the interferometric channels and are unavoidable in planar, integratedoptical, multi-baseline beam combiners. Following this approach, three telescope beam combiners for the mid infrared $(\lambda \sim 10 \mu \mathrm{m})$ were manufactured in gallium lanthanum sulfide (GLS), a chalcogenide glass with transparency window extending to the mid infrared, and tested in the laboratory (Ródenas et al. 2012). More recently L-band, ABCD combiners for two telescopes and with cross-over avoidance were manufactured and characterized in the same glass (Diener et al. 2017). A more radical use of the 3D structuring of ULI was demonstrated by the introduction of the so called discrete beam combiners (DBC Minardi and Pertsch 2010), which exploit the propagation of light in two-dimensional, periodic arrays of evanescently coupled waveguides to combine interferometrically several beams (see Fig. 9 for an example). An advantage of this scheme is the avoidance of losses due to bent waveguides and waveguide cross-overs, as well as the possibility to build very short devices (typically two coupling lengths). R-band three-telescope combiners were tested in the lab with monochromatic (Minardi et al. 2012) and polychromatic light (Saviauk et al. 2013), while a $2.5 \mathrm{~cm}$-long prototype of a 4-telescope combiner for L-band was reported to retrieve accurately phase and visibility of pairs of input beams (Diener et al. 2017).

In the presence of atmospheric turbulence, model-free interferometric imaging requires at least the combination of triplets of telescopes to recover partial phase information from the interferograms (Rogstad 1968). Additionally, a dense sampling of the Fourier plane of the object image should be achieved by adding many different baseline-triplets, a goal which is essentially accomplished in two different ways. Either the telescope-triplets are relocated to form more baselines, or a large array of telescopes is combined at once. While the first approach improves the sensitivity, the second approach allows the rapid retrieval of the visibilities 
avoiding the time overheads required to relocate the telescopes. The development of multiple-telescope beam combiners is therefore indicated for the imaging of rapidly varying astronomical targets such as novae (Chesneau et al. 2008) or planet transits (Kloppenborg et al. 2010). While for the time being only up to 6 telescopes may be combined at existing facilities (CHARA), plans for new facilities featuring 10 (Buscher et al. 2013) or 20 telescopes (Kraus et al. 2016) have been proposed. CHARA is currently developing a new beam combiner in K-band which will allow to combine 3 quadruplets out of the 6 telescopes using spare components of the GRAVITY combiner (ten Brummelaar et al. 2016). Besides the already mentioned 4-telescope combiner for mid infrared (Diener et al. 2017), beam combiners featuring 9 input channels in $\mathrm{H}$-band and active control of the optical path have been developed for aperture masking techniques (Martin et al. 2016) (see next section). As may be evident from the previous text, most of the integrated optics beam combiners were developed in the near-infrared band due to the availability of the very mature silica-based technology, which has been developed for the telecommunication market. However, the astrophysical characterization of a target often requires the analysis of starlight over a wide spectral range, therefore ongoing research in interferometric instrumentation is focusing on devising alternative materials and technologies to extend the spectral coverage of integrated optics beam combiners. In particular, both the booming interest towards cold targets such as debris disks and exoplanets (Kraus and Ireland 2012) and the difficulty to perform interferometry at short wavelength motivated research aimed at achieving beam combination in the mid-infrared. The manufacturing of integrated optics combiners for MIR wavelengths (Labadie et al. 2008) requires the development of technological platforms allowing the micro-structuring of materials with transparency extending beyond the $2.4 \mu \mathrm{m}$ cut off of silica glass (Labadie and Wallner 2009). As such, the research in the field has started to accelerate in the past few years, as these technology platforms began to become available, also thanks to the growing biophotonics market. A paradigmatic technology platform is laser writing. This platform employs tightly focused, intense laser beams to modify locally the refractive index of glasses, an thus create light guiding structures. Both continuous wave lasers as well as high repetition rate femtosecond lasers have been employed to manufacture $2 \times 2$ directional couplers for MIR in chalcogenide (Labadie et al. 2011; Tepper et al. 2017) and zirconium barium lanthanum fluoride glasses (ZBLAN) (Gross et al. 2015; Tepper et al. 2017). As mentioned before, threedimensional 3-telescope (Ródenas et al. 2012) and 4-telescope (Diener et al. 2017) beam combiner prototypes were manufactured for respectively the $\mathrm{N}$ and the $\mathrm{L}$ band using ultrafast laser writing in Gallium Lanthanum Sulfide. Recently, chalcogenide planar integrated optics manufactured by conventional photolithographic techniques delivered waveguides with extremely low losses ( $\sim 0.3 \mathrm{~dB} / \mathrm{cm}$; Ma et al. 2013$)$ and opened the perspectives for complex beam combiners for astronomical use. In this context, highly achromatic directional couplers based on multi-mode interference couplers (MMI Besse et al. 1994) were manufactured and tested (Kenchington Goldsmith et al. 2016, 2017b) based on a design optimized for applications to nulling interferometry (Kenchington Goldsmith et al. 2017a). 
Aperture masking Aperture masking is a high-resolution imaging technique based on interferometric principles. In its basic form it consists of masking the pupil of the telescope with sub-apertures smaller than the typical correlation length of the turbulent atmosphere (the Fried parameter; Fried 1966). If the sub-apertures are chosen so that the lines connecting each possible pair (baselines) are unique (a so called non-redundant arrangement), the short-exposure (less than the atmospheric coherence time) intensity pattern in the focal plane of the telescope is a twodimensional interference pattern from which it is possible to measure the visibility function of the astronomical target and thus retrieve its angular irradiance distribution. First proposed by Fizeau for two sub-apertures (Fizeau 1868) and extended to multiple apertures by Rhodes and Goodman (1973), the technique was tested on-sky in the mid-1980s by Baldwin et al. (1986), who were able to reconstruct the image of a binary star soon afterwards (Haniff et al. 1987).

While aperture masking can reach an angular resolution of $\lambda / 2 D, D$ being the aperture of the telescope, most of the light of the astronomical target is rejected by the mask, limiting the application of the method to relatively bright sources, which deliver high signal-to-noise interference patterns even with short integration times. To overcome this limitation, Lacour et al. (2007) proposed the pupil-remapping technique, in which single-mode optical fibers are used to remap a densely sampled pupil plane into a non-redundant arrangement of diffraction-limited sources which are converted into an interferogram by a lens. In this configuration, all the light collected by the telescope can be used and the redundant baselines are measured without loss of contrast. Because of the potentially large number of baselines measured within the pupil of the telescope, a further advantage of pupil remapping is the possibility to retrieve images with high dynamic-range and thus be able to observe nearby exoplanets. A first on-sky demonstration of the pupil remapping concept came with the instrument FIRST (Huby et al. 2012). FIRST used 9 microlens-coupled, single-mode optical fibers to transform a redundant, twodimensional array of sub-apertures of the $3 \mathrm{~m}$ telescope at the Lick observatory into a non-redundant linear array of beams. A fringe pattern was then imaged on a camera by focusing the beams with an anamorphic optical system. The instrument operated in the R-band and used a prism to obtain spectrally resolved visibilities $(R \sim 300)$. Short exposures of the fringes were possible thanks to a EMCCD detector. Closure phases with standard deviation well below $1^{\circ}$ were obtained by averaging 100 frames (standard deviation without averaging $5^{\circ}$ ). FIRST was later upgraded to allow the combination of two separate sets of 9 sub-apertures and used to study the Capella binary system (Huby et al. 2013). A miniaturization of the pupil remapping setup was proposed and realized with the Dragonfly project (Tuthill et al. 2010), led by the Australian Astronomical Observatory (AAO) team. Instead of using optical fibers to remap redundant sub-apertures in a non-redundant array, the Dragonfly instrument used laser writing to fabricate a compact 2D to 1D pupilremapper in a silica glass substrate (Charles et al. 2012) (see Fig. 10). Besides reducing the size of the remapping function, the integrated optical component allowed a stabler operation of the instrumental transfer function. The assembled instrument operated in the J-and H-band and tested on sky at the 4-m telescope at AAO (Jovanovic et al. 2012). 


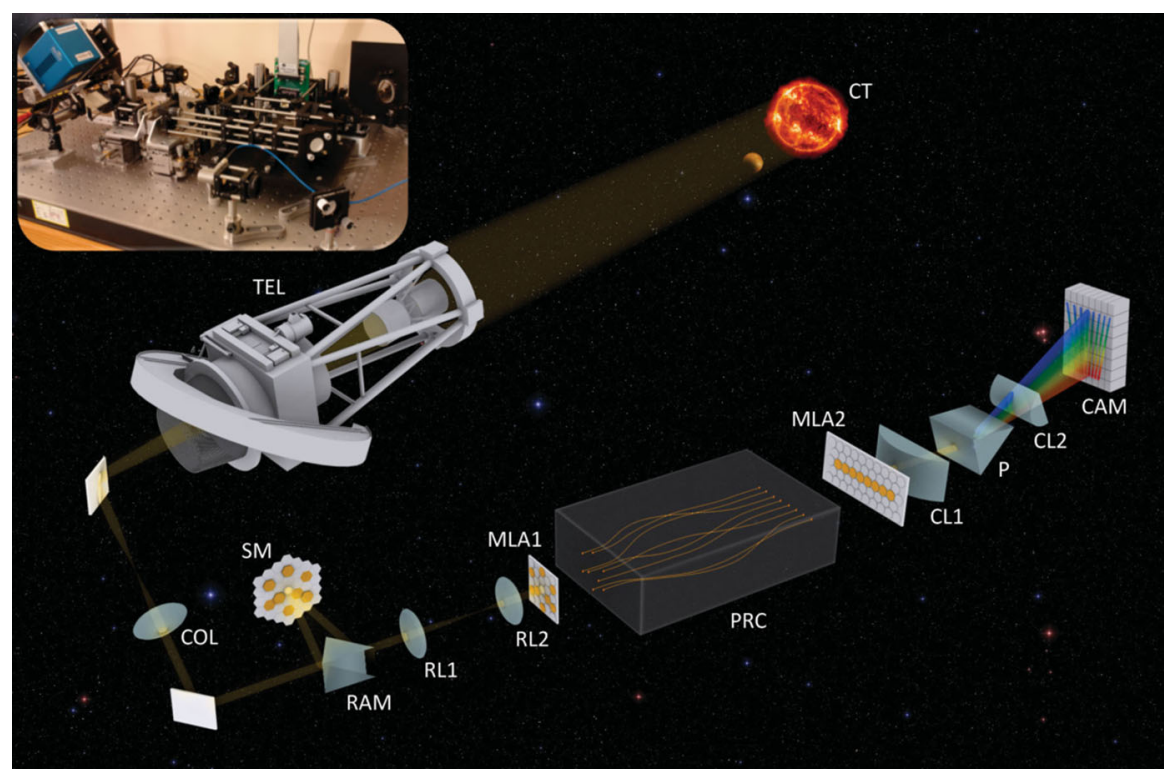

Fig. 10 Scheme of the Dragonfly instrument. The light from the telescope (TEL) is collimated (COL). In the pupil plane an array of micromirrors (SM) redirects selected sub-apertures to an integrated optical component (PRC - injection in the waveguides is allowed by the microlens array MLA1). In the PRC, three-dimensional waveguides are used to carry light from a two-dimensional arrangement of input sites to a non-redundant linear array of outputs. Output light is collimated by microlenses (MLA2) and combined and dispersed with a free-space anamorphic optical system (CL1-P-CL2) on a detector (CAM), where interference fringes are recorded. Image reproduced with permission from Jovanovic et al. (2012), copyright by the authors

The best experimental fringes were obtained on Antares by combining 4 of the 8 possible sub-apertures. The used detector (an InGaAs camera) had a high noise level so that only exposures much longer than the coherence time of the atmosphere were recorded. This led to a significant reduction of the measured fringe visibility. Nonetheless, a performance similar to the FIRST instrument (standard deviation of the closure phase $\sim 5^{\circ}$ ) was obtained.

Research in astrophotonic aperture masking is currently focusing on the development of hybrid devices allowing the combination of the sub-apertures in an integrated optics beam combiner, which could deliver higher sensitivity than multi-axial combination (Minardi et al. 2016). A fiber-fed, 9-aperture device operating in the visible and including a lithium niobate phase modulator was recently assembled in the laboratory in the frame of the FIRST/SUBARU project (Martin et al. 2016). This device could be used to scan fringes as well as delivering nulling capabilities to the device (see Sect. 4.4). While the proof-of-concept prototype demonstrated the functional operation of the device, losses occurring at the interfaces between different units (fiber/beam splitter, beam splitter/modulator, modulator/beam combiner, beam combiner/fiber) were believed to be mainly responsible for the high insertion losses of the device $(\sim 30 \mathrm{~dB})$. 


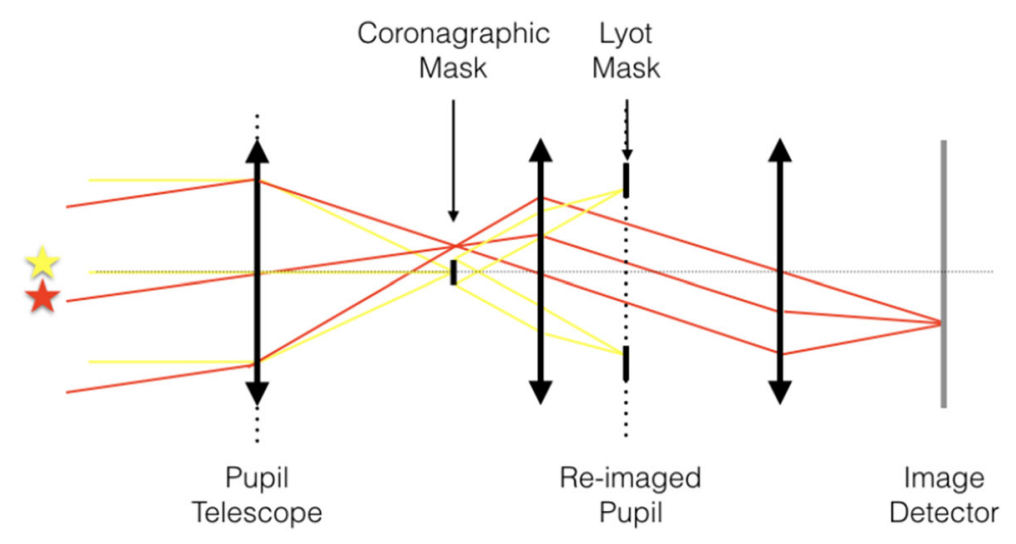

Fig. 11 Scheme of the Lyot coronagraph. Light from the main star (yellow) is attenuated by the beam stop in the focal plane and the Lyot mask in the pupil plane. In contrast, light from an off-axis source (red) propagates mostly unattenuated through the optical system and is recorded by the detector

\subsection{High-contrast imaging}

Coronagraphic phase plates Coronagraphy owes its name to an instrument invented by Bertrand Lyot in the 1930s to create artificial solar eclipses for the observation of the corona, which has a brightness several orders of magnitude below that of the photosphere. The instrument consisted in a focal plane stop (an absorbing disk of diameter matched in size to the image of the photosphere) followed by an optical spatial filter in the pupil plane (the so called Lyot filter) required to suppress light diffracted by the spider and the secondary mirror of the telescope (see Fig. 11). A modified version of the coronagraph was developed at the beginning of the 1980s to deliver images of faint objects close to stars, such as low-mass companions and protoplanetary disks. The first stellar coronagraph used an occulting mask in the focal plane of the telescope followed by a Lyot mask (see Vilas and Smith 1987 for a description of the instrument). This instrument was used for the landmark discovery of the protoplanetary disk surrounding $\beta$ Pictoris (Smith and Terrile 1984). Interest in stellar coronagraphy increased in the last 25 years as soon as effective AO systems became available and the quest for direct observation of extrasolar planets became a mainstream goal in astrophysical research.

A particularly important result, which opened coronagraphy to the field of photonics, was the proposal of using phase plates instead of absorbing mask as field stops in the coronagraphs (Roddier and Roddier 1997). The rationale of the method consists in reversing the sign of a portion of the central lobe of the point spread function of a highly corrected aperture (e.g., a space telescope). In the pupil plane, the phase inverted portion of the PSF interferes destructively with the starlight, which is scattered outside the pupil, where it can be eliminated by a circular aperture matching the pupil diameter. In practice the phase mask acts as a mode converter, transforming the PSF of the AO-corrected telescope into an annular beam. The advantage of phase masks over traditional beam stops is that the inner working angle of the coronagraph (i.e., the minimal distance at which a faint 
companion can still be detected amid the PSF of the attenuated star) is close to the diffraction limit. An experimental verification of the concept was attempted in the laboratory a few years later (Guyon et al. 1999), the mask being manufactured by etching a small cylinder protruding from a glass plate with a height sufficient to induce a $\pi$ phase shift at a wavelength of $633 \mathrm{~nm}$. The coronagraph showed a peakto-peak attenuation of the monochromatic point spread function by a factor $1 / 16$, far worse than the expected value of $10^{-4}$. Most of the loss of contrast was due to the round-off of the edges of the phase shifter resulting from the fabrication process.

An evolution of the concept of phase mask coronagraph is the vortex coronagraph. Similar to quantum mechanical wavefunctions (Berry 1984), optical vortices are electromagnetic waves nesting a phase singularity, i.e., a point in the transverse plane of the optical field where the phase is undefined and the amplitude vanishes. Around the singularity, the phase of the field grows linearly with the azimuthal angle $\theta$. Because of the continuity of the optical field, the phase difference between $\theta \rightarrow 2 \pi$ and $\theta \rightarrow 0$ should be an integer multiple of $2 \pi$ (this multiple being known as the topological charge), so that the phase ramps can take only discrete values of their azimuthal gradient. The name vortex is derived from the fact that the phase gradient whirls around the central singularity in a fashion resembling the velocity field of a vortex in a liquid (see Fig. 12). Alternatively, we can visualize the optical vortex as a wave with an helical wavefront, the twist of which can assume only discrete values.

A remarkable mathematical result is that an ideal lens can transform an Airy disk nesting an optical vortex of even topological charge into a light distribution which vanishes exactly within the area of the pupil (Foo et al. 2005). This effect can be exploited in coronagraphy by designing a phase mask with an engraved helical ramp (also described as vortex lens) and shaping the Lyot mask as a circular aperture. The first demonstration in the lab of a vortex coronagraph was reported in Lee et al. (2006), where a peak-to-peak attenuation of 0.05 was measured. An improved phase mask was later manufactured (monochromatic attenuation in the lab up to $2 \cdot 10^{-3}$ ) and tested on-sky with a high Strehl, clear aperture, test telescope (diameter 1 inch)
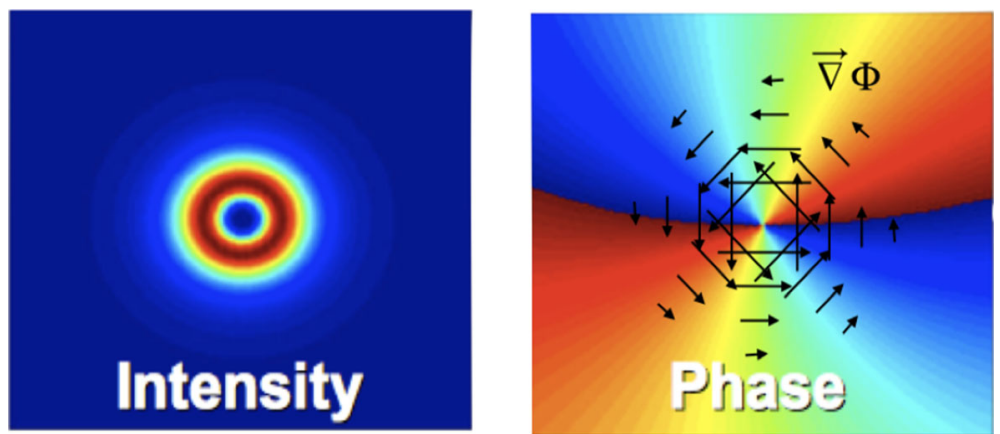

Fig. 12 Intensity (left) and phase map (right) of an optical vortex. The phase is undefined in on the axis, where the optical field vanishes. The transverse gradient of the phase (black arrows) rotates around the optical axis in analogy to the velocity field of a vortex in a liquid. The transformation of the PSF of an AO corrected telescope into a vortex is exploited to reject the light of a star and image its faint companions 
(Peters et al. 2008). In the latter experiment, an average attenuation of $3 \%$ at visible wavelength (20 $\mathrm{nm}$ bandwidth) was attained, most of the contrast loss being attributed to the chromatic dispersion of the phase mask.

An inherent problem of phase masks is indeed that they work only at the design wavelength, as the phase delay introduced by a plate of fixed thickness scales as $\lambda^{-1}$. This effect can be mitigated by designing a vortex lens composed of two layers made of materials with different dispersive properties (Swartzlander 2005, 2006). The inherent complexity of the fabrication of such masks prevented however an experimental demonstration of the concept so far. A different approach was adopted by Errmann et al. (2013), which adapted a concept of achromatic vortex generation with ultrashort laser pulses to coronagraphy (Bezuhanov et al. 2004). In this case, the vortex beam was imprinted on the PSF of a beam by an off-axis, blazed computer generated hologram $(\mathrm{CGH})$, which unavoidably disperses broadband light. The dispersion was compensated by diffracting the vortex beam with an ordinary phase grating with the same periodicity of the $\mathrm{CGH}$ but opposite orientation of the blazing. Peak-to-peak attenuations of the PSF better than $10^{-3}$ where demonstrated in the laboratory for a bandwidth covering most of the R-band.

An alternative to conventional phase masks for vortex coronagraphy is represented by birefringent optical plates, which modify locally the vectorial properties of light and generate polarization vortices. Polarization vortices are optical fields in which the local linear polarization vector is oriented at an angle proportional to the azimuthal angle. Linearly polarized light beams can be converted into polarization vortices by a birefringent medium which locally acts as a $\lambda / 2$ retardation plate. An early example is a glass plate coated with liquid crystals, whose slow axes are oriented and polymerized by a UV laser beam (Mawet et al. 2009). If the angle of the slow axis direction is equal to the azimuthal angle, then the field in the circularly polarized base exhibits an helical wavefront of topological charge +2 and -2 for the left- and right-hand polarization states, respectively (Mawet et al. 2005). The far-field of these vectorial fields has the same light distribution as the scalar vortices of topological charge $m=|2|$ and thus can be used in coronagraphs with a Lyot mask with full aperture slightly smaller than the pupil. A test on sky of the vectorial vortex coronagraph manufactured with liquid crystal technology was reported in 2010 (Serabyn et al. 2010). The well corrected 1.5 meter aperture of the Hale telescope at Palomar (Serabyn et al. 2007) was used to deliver to the phase plate a beam in $\mathrm{K}_{\mathrm{s}}$-band with Strehl ratio exceeding $90 \%$. A raw peakto-peak attenuation of $\sim 1 / 50$ was reported over a band of $14 \%$, which was sufficient to detect three of the planets surrounding HR8799 after electronic removal of the attenuated PSF of the telescope. Chromatic dispersion of the retardation plate was shown in the lab to contribute significantly to the loss of contrast in the coronagraph (Mawet et al. 2010), a problem that could be solved by the use of subwavelength gratings (Mawet et al. 2005). These so called annular groove phase masks (AGPMs) consist of concentric grooves with sub-wavelength spacing. The sub-wavelength spacing of the grooves prevents light diffraction and acts as an effective birefringent medium (Born and Wolf 1997) with an azimuthally varying orientation of the slow (fast) axis. The shape and depth of the grooves is chosen so 
that the retardation between the slow and the fast axis corresponds to $\lambda / 2$. Two distinct technologies have been used to manufacture AGPMs, namely 1) the etching of grooves in diamond and 2) auto-cloning of photonic crystal structures. The first technology was optimized for MIR coronagraphy in L-band and could deliver broadband peak-to-peak attenuations at the $10^{-3}$ level in the laboratory (Delacroix et al. 2012, 2013; Vargas Catalán et al. 2016). Test on-sky of these phase masks were also successfully accomplished at the VLT (Mawet et al. 2013) and the Keck telescopes (Serabyn et al. 2017), but delivered a raw attenuation at the $10^{-2}$ level, mainly due to leakage of starlight from the central obstruction of the telescope and residual tip-tilt of the AO system. Regarding the second technology, a vortex coronagraph based on photonic crystals (i.e., sub-wavelength periodic two- or threedimensional assemblies of materials with different refractive index) with axial symmetry was manufactured for the visible band (Murakami et al. 2013) and tested in the lab with circularly polarized light. A raw peak-to-peak attenuation at the $10^{-4}$ level was achieved in the lab with a clear circular pupil in a band ranging from $\lambda=543 \mathrm{~nm}$ to $\lambda=633 \mathrm{~nm}$ wavelength. Analysis pointed out that the attenuation was mainly limited by the chromatic dependence of the birefringence phase shift in the vortex plate. No test on sky has been reported so far for this phase mask.

A discretized version of the vortex coronagraphs is represented by the 4-quadrant and 8-octant plates. In practice, the azimuthal phase ramp is simply approximated by 0 and $\pi$ phase shifting regions which alternately divide the mask plane in 4 (4quadrant mask Rouan et al. 2000) or 8 (8-octant mask Murakami et al. 2013) equal sectors. The 4-quadrant mask is equivalent to a vortex with topological charge $m=2$, while the 8-octant mask to a topological charge of $m=4$. The masks work similarly to the vortex phase plates with the exception that the discovery space is limited by shadow areas extending along the interfaces between the 0 and $\pi$ shift sectors, where the throughput of an off-axis companion is significantly decreased. Silica 4-quadrant masks designed for Ks-band and manufactured by silica layer deposition techniques saw the first light at the VLT-NACO instrument in 2004 (Boccaletti et al. 2004) and delivered broadband attenuations of the PSF larger than $\sim 10$, reaching a factor $\sim 100$ with narrow band filters. The poor attenuation in broadband was mainly due to the intrinsic chromatic behavior of the $\pi$-phase-shift layer, as well as the presence of the secondary mirror. A more achromatic behavior was achieved in the lab with the 8-octant polarizing plate, which used the same photonic crystal structure mentioned above for the AGPM coronagraph. Tests of the 8-octant plate with monochromatic, linearly polarized light from a clear pupil revealed peak-to-peak attenuations $<10^{-5}$ at the wavelengths of $\lambda=532 \mathrm{~nm}$ and $\lambda=633 \mathrm{~nm}$, the attenuation being apparently limited by the speckles induced by imperfections in the optics of the setup. The on-sky test of an H-band 8-octant phase mask is foreseen at the SCExAO facility of the Subaru telescope in the near future (Jovanovic et al. 2015).

Beyond the design and manufacturing issues, the ultimate starlight rejection of phase mask coronagraph depends on the features of the telescope and its AO system, as well as the angular extent of the attenuated star (Guyon et al. 2006). In particular, the presence of a secondary mirror in the pupil of the telescope creates a circular 
pattern of light nested in the pupil of the Lyot mask, which carries a power fraction of the starlight equal to the fractional area of the secondary (Jenkins 2008). The simplest approach suppressing this light is to include an oversized secondary stop in the Lyot mask, which however limits the throughput of the coronagraph and does not reject completely the light scattered by the secondary mirror. A more advanced solution is to use pupil apodization techniques (i.e., transform the PSF in a fastdecreasing, ring-less light distribution such as a Gaussian beam Guyon 2003) by means of aspherical mirrors/lenses or transmitting apodizers before the vortex phase mask. A concept was recently proposed to apodize the pupil with 3-level stepped transmission plates which would cancel perfectly the light profile of the secondary within the pupil of a vortex coronagraph (Mawet et al. 2013). The SCExAO team at the Subaru telescope is currently developing suitable pupil apodizing lenses to remap a pupil with secondary into a clear aperture (Lozi et al. 2009), before focusing the starlight onto a phase mask coronagraph (Jovanovic et al. 2015). A second issue on achievable contrast is related to the quality of the AO correction. High Strehl ratios and low residual tip-tilt error are mandatory with the use of vortex phase masks with topological charge $m=2$, a condition somewhat relaxed by vortex plates of higher topological charge at the expense of increasing the inner working angle of the coronagraph (Guyon et al. 2006). Various techniques for pupil plane tip-tilt sensing and correction have been developed in recent years to keep the pointing stability of the telescope below the few mas level (Guyon et al. 2009; Huby et al. 2017). Qualitatively similar to a tip-tilt error, a partially resolved star will leak light in the coronagraph and reduce the contrast, an effect which affects more dramatically phase masks with smaller inner working angle (Guyon et al. 2006). However, a measurement of the maximal attenuation depth with $m=2$ vortex coronagraphs could be conversely used to estimate precisely the angular diameter of the target star (Ruane et al. 2014).

Integrated nulling interferometers Nulling interferometry is a high-contrast technique, which is the analogue of coronagraphy for stellar interferometers. The technique exploits the phase difference of fringes originating from on-axis sources respect to off-axis ones (Bracewell 1978). By locking the interferometer to a dark fringe, the light of the on-axis source (e.g., a parent star of an exoplanet system) will be rejected to the bright channel of the beam combiner, while the light of a dim offaxis companion (e.g., an exoplanet) would be transmitted by the dark port. In practice the quantity of interest in this technique is the null depth, defined as the ratio between the flux measured at a dark fringe and the corresponding bright fringe. The null depth in a planet-star system will be dependent on the separation of the two bodies (which can be retrieved varying the orientation and length of the baseline) and their relative brightness in the wavelength band of interest. For earth-like planetary systems the null depth is required to be as high as $\sim 10^{-6}$ in the N-band.

So far, nulling interferometry has been implemented at the MIR N-band by means of conventional optics at the Keck Interferometer (Serabyn et al. 2012) and the Large Binocular Telescope Interferometer (LBTI) (Defrère et al. 2016). The Keck Interferometer completed a survey of the exo-zodiacal dust level of about 50 stars (Millan-Gabet et al. 2011; Mennesson et al. 2014b), while a comprehensive 
overview of the results of the LBTI survey has recently been published (Ertel et al. 2020).

Astrophotonic nulling interferometry has mainly been the object of laboratory research, with the exception of the on-sky operation of a near-infrared nuller prototype at the Palomar 200" telescope, which exploited a single-mode optical fiber as spatial filter (Martin et al. 2008; Hanot et al. 2011). The instrument (Palomar Fiber Nuller) consisted in two rotating apertures in the pupil plane of the telescope, which were optimally focused by a lens on the tip of the fiber, so that when the null of the fringes is centered in the fiber axis, a negligible amount of light would be coupled in the fiber (Haguenauer and Serabyn 2006). An important driver for nulling experiments has been the feasibility study of for the TPF-I (Breckinridge and Lindensmith 2005) and Darwin (Fridlund 2004) space nulling interferometer missions, which were later canceled. A high-contrast integrated optics 2-channel beam combiner for near-infrared demonstrated the possibility to reach a nulling depth of $3 \cdot 10^{-5}$ with monochromatic light and $2 \cdot 10^{-4}$ with polychromatic light with a bandwidth of $80 \mathrm{~nm}$ (Weber et al. 2004). A fiber nuller laboratory experiment with MIR laser $(\lambda=10.6 \mu \mathrm{m})$ beams focused on a single-mode conductive waveguide demonstrated a few years later an extinction ratio at the $6 \cdot 10^{-5}$ level (Labadie et al. 2007). The possibility to combine MIR beams and modulate the relative phase on a single integrated optics chip was also explored. An active twoway titanium indiffused lithium niobate beam combiner was designed for L-band and tested in the laboratory (Hsiao et al. 2009). The device featured an insertion loss of $4.7 \mathrm{~dB}$ and an on-chip fringe scan of broadband $(\Delta \lambda=500 \mathrm{~nm})$ light at $\lambda=$ $3.4 \mu \mathrm{m}$ was demonstrated. However, the measurement could not asses the visibility and the nulling depth of the fringes.

Renewed interest for photonic nulling interferometry rose in recent years, stimulated by the possibility to image exoplanetary systems with interferometric techniques (Kraus et al. 2016). In this context, we mention the a proof-of-principle experiment of an integrated optics 4-telescope nulling beam combiner (Errmann et al. 2015) based on a scheme devised by Angel and Woolf (1997), and the aforementioned realization of an integrated optics $2 \times 2$ multimode interference coupler in chalcogenide glass (Kenchington Goldsmith et al. 2017b), which was designed to achieve a deep broadband nulling level in L-band (Kenchington Goldsmith et al. 2017a).

More recently an H-band 2x2 coupler manufactured by means of femtosecond laser writing (Norris et al. 2014) and integrated at the SCExAO testbench of the Subaru telescope on Mauna Kea was tested. The device (named GLINT Norris et al. 2020) was used to combine interferometrically two subapertures of the telescope separated by 5.5 meters. The phase difference between the two channels was locked to obtain a null in one of the two interferometric outputs of the device. Due to the residual fluctuation of the piston at each subaperture and the varying coupled flux in the two input waveguides, a statistical retrieval of the nulling depth has been used (Hanot et al. 2011). A time series of the estimated null depth was used to build a statistical distribution of their values, which could be fitted by an appropriate model. To test the device, several stars with known diameter, which were partly resolved by 
the telescope baseline, have been measured. The fitted null depths of the stars ranged from 0.0083 to 0.18 with a precision in the order of $10^{-4}$. The estimated star diameters corresponded within 1 mas with those measured with long-baseline interferometry. Upgrades of the instrument to more baselines is foreseen in the near future.

\subsection{Metrology and calibration}

Laser guide stars AO systems require light from a bright star to drive the wavefront sensor, resulting in a limited sky coverage of diffraction limited imaging. To overcome this limitation, (Foy and Labeyrie 1985) proposed to generate an artificial reference star in the sky using a laser, an idea derived from LIDAR experiments which are used to investigate the structure of the atmosphere by recording backscattered light from a laser beam directed towards the sky. Two types of Laser Guide Stars (LGS) were discussed in this seminal paper, one relying on Rayleigh scattering by low atmospheric layers, and one utilizing resonant scattering of sodium atoms.

Rayleigh LGSs use powerful pulsed visible laser sources and a synchronized time gated wavefront sensor to isolate the backscattered photons from a particular layer of the lower atmosphere. The configuration of the deformable mirror and the exposure of the science camera must occur within the coherence time of the atmosphere. The first experimental demonstration of this type of LGS was indeed achieved at the end of the 1980s by the US Air Force working on the development of the space shield Star Wars program (Primmerman et al. 1991). Today, the evolution of Rayleigh LGS is used in astronomy mainly to compensate ground layer turbulence (first 10-20 km) as the power of the scattered light decreases rapidly with the altitude (Rabien et al. 2019).

LGS systems based on the detection of resonant scattering from sodium atoms use a laser tuned at the wavelength of the $D_{2}$ component of the sodium doublet to excite the sodium layer at an altitude of about $93 \mathrm{~km}$. The exact altitude can vary by a few kilometers according to the season and atmospheric conditions. Sodium is found in this layer because of an equilibrium between the intake of meteoric debris and depletion by chemical reactions happening at lower altitudes. The laser beam is usually launched off the axis of the telescope/wavefront sensor to separate geometrically Rayleigh scattered light from the lower layers of the atmosphere.

The development of powerful laser sources suitable for LGS systems is an important aspect of astrophotonics, which has seen a rapid progress in recent years. Here we review only the development of sources for sodium layer LGSs, as research in the field was mainly motivated by astronomical applications.

Because powerful sodium line solid-state lasers are tricky to build (see below), the first examples of sodium LGS used dye solutions as lasing medium. These lasers are tunable over the fluorescence band of the dye molecules, which are excited by flashlamps or other lasers tuned at their absorption band. A dye laser usually consists in a low-power master oscillator which seeds one or more poweramplification stages. The selection of the longitudinal mode is accomplished in the 
master oscillator by an etalon. A solution of Rhodamine 6G in ethylene-glycol pumped by $4 \times 10 \mathrm{~W}$ frequency doubled, continuous wave Nd:YAG lasers $\left(\lambda_{0}=532 \mathrm{~nm}\right)$ was the lasing medium employed by PARSEC, an early sodium LGS at the European Southern Observatory (ESO) VLT, which yielded about $12 \mathrm{~W}$ of continuous radiation at $589 \mathrm{~nm}$ (Bonaccini et al. 2002).

Research on solid-state laser sources progressed as well, the basic technological solution being the exploitation of the capability of neodymium-doped garnet (Nd:YAG) to deliver laser transitions at $\lambda_{1}=1064 \mathrm{~nm}$ and $\lambda_{2}=1319 \mathrm{~nm}$. Radiation at a wavelength of $589 \mathrm{~nm}$ can be generated by mixing in a nonlinear crystal two pump lasers oscillating at $\lambda_{1}$ and $\lambda_{2}$, a process known as sum frequency generation. Jeys and co-workers first explored the possibility to mix two Nd:YAG Q-switched lasers to create a sodium line source for astronomical applications (Jeys et al. 1989). Intracavity etalons with a tunability of about $0.5 \mathrm{~nm}$ were used in the lasers to select the lasing wavelength, while the sum frequency was obtained by a single pass in a $5 \mathrm{~cm}$-long lithium niobate crystal. The source yielded up to $395 \mathrm{~mW}$ of radiation at $589.159 \mathrm{~nm}$ with a pulse repetition rate of $1 \mathrm{kHz}$. Because of the relatively low power, the source was used only in LIDAR applications. Since this first experiment, considerable progress has been made to develop powerful sources for sodium LGSs based on Nd:YAG sum frequency generation, both in the pulsed and continuum operation regime. A $20 \mathrm{~W}$ continuum source based on $\mathrm{Nd}$ :YAG injection-locked lasers at $\lambda_{1}$ and $\lambda_{2}$ feeding a doubly resonant, sum-frequency cavity was demonstrated by Bienfang et al. (2003). The final engineered source (Frequency-Addition Source of Optical Radiation - FASOR) achieved $50 \mathrm{~W}$ of output power at the sodium line and was eventually installed at the $3.5 \mathrm{~m}$ telescope of the Starfire Optical Range facility of the US Airforce, where it was used for adaptive optical imaging of satellites. Multiwatt, pulsed Nd:YAG sources are currently employed at the Subaru (Saito et al. 2010), Palomar (Hankla et al. 2006), Keck I and Gemini South observatories (Fig. 13). In these cases two synchronized mode-locked Nd:YAG lasers are used and mixed in single pass in a periodically poled $\mathrm{Mg}-\mathrm{O}$-doped stoichiometric lithium tantalate (PPMg-O:SLT) crystal (Saito et al. 2007). Synchronization of the lasers is achieved by a phase shift of the radiofrequency drivers of the loss modulators inside the cavities. Output powers of $6.8 \mathrm{~W}$ were reported as well as a power stability of $2.2 \%$ over 8 hours.

The complexity of the sodium laser systems requires trained personnel and controlled environments for their correct operation. The need for a more rugged and turn-key sodium line source motivated ESO and the company TOPTICA to develop a fiber-based sodium line LGS. Fiber lasers can deliver high power with excellent beam profile and require no alignment of the laser cavity, which is formed by spliced integrated components. The fiber sodium line laser used a $70 \mathrm{~W} \mathrm{Yb-doped}$ fiber laser operating at $1020 \mathrm{~nm}$ to amplify through the Raman process a $\lambda_{0}=$ $1178 \mathrm{~nm}$ seed from a diode laser in a $100 \mathrm{~m}$-long nonlinear fiber (Feng et al. 2008, 2009). Radiation at $589 \mathrm{~nm}$ was obtained by frequency doubling in a phaselocked singly resonant cavity containing a lithium triborate crystal (Feng et al. 2009). Output powers of $50 \mathrm{~W}$ at the sodium line wavelength were achieved by combining interferometrically three Raman fiber lasers at $1178 \mathrm{~nm}$ (Taylor et al. 2010). The system engineered by TOPTICA includes several servo systems 


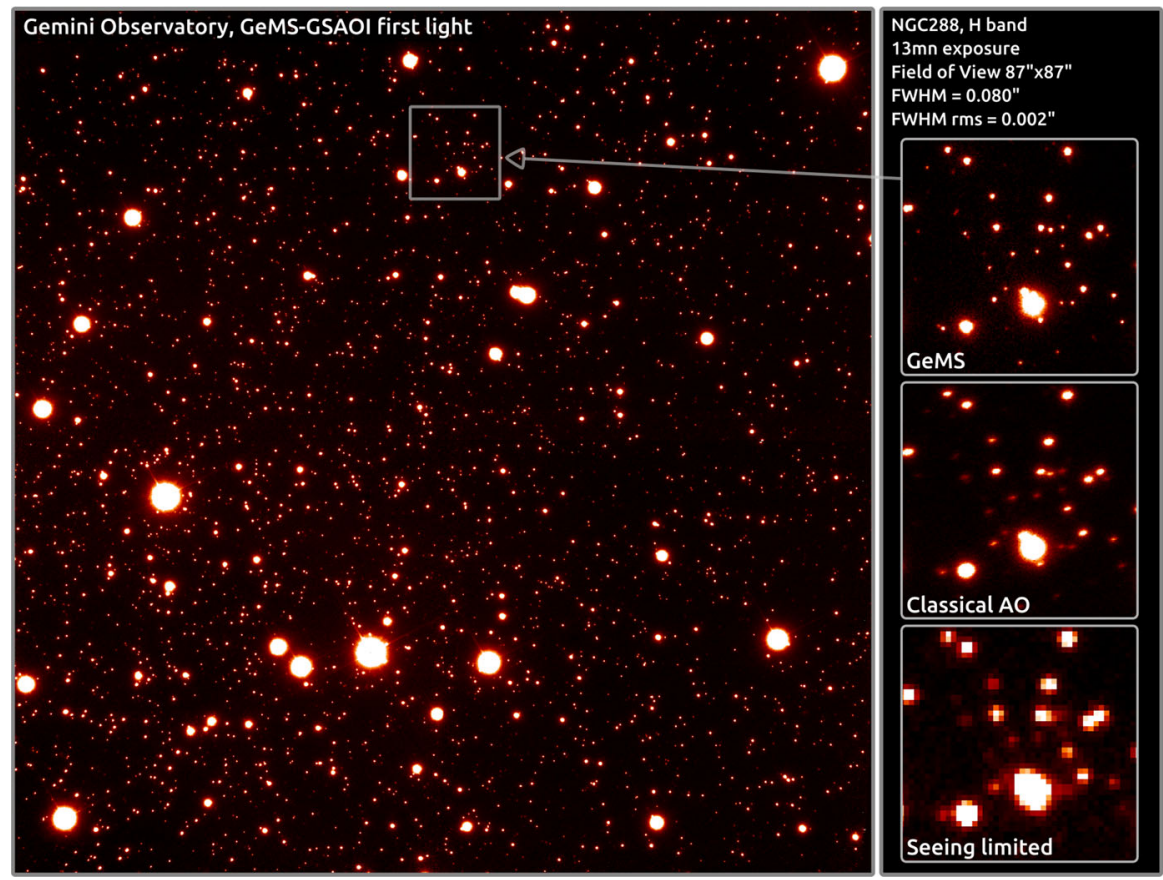

Fig. 13 Active photonic devices such as lasers are becoming a working instrument for astronomers. Laser guide stars extend the use of Adaptive Optics (AO) to targets which are too faint to use natural guide stars to lock the AO system. The picture shows an $87 \times 87$ arcsecond portion of the cluster NGC288 taken with help of Gemini South's GeMS/GSAOI $589 \mathrm{~nm}$ laser guide star facility. The image is taken at 1.65 microns ( $\mathrm{H}$ band) and features an average full-width at half-maximum resolution of slightly below 0.080 arcsecond. The insets on the right show a comparison of the same close up of the cluster taken with LGSAO (top), with natural guide star AO (middle), and seeing-limited observations (bottom). (Credit: Gemini Observatory)

enabling a power stability better than $2 \%$ over several hours and a fully maintenance-free operation (Arsenault et al. 2012). Four units of the laser sources are currently forming the laser guide star asterism of the LGS facility at the UT4 VLT (Calia et al. 2004), which feeds the GRAAL/HAWK-I (Paufique et al. 2012) and the GALACSI/MUSE (Ströbele et al. 2012; Laurent et al. 2010) instruments.

Astrocombs As we have discussed in the introduction, the key for the future challenges of high-resolution spectroscopy lies in the capability to calibrate accurately the spectrograph. This is usually achieved by means of standard lamps exciting the emission lines of heavy elements such as Thorium and Argon (Baranne et al. 1996), or by filtering starlight or a white light source with a gas cell filled with iodine vapor (Butler et al. 1996). While the short time accuracy of the position of the lines of Thorium Argon (ThAr) lamps is at the few cm/s level (Wilken et al. 2012), their uneven frequency and brightness distribution does not allow for a uniform calibration accuracy across the spectral range of the spectrograph. Moreover, aging of the emission lamps reduces the accuracy on a time scale of several hundred hours of usage (Mayor et al. 2009). An additional limitation of 
atomic references is the unavailability of satisfactory standards for mid infrared bands (Seemann et al. 2014).

In the past decade, interest has been mounting for the realization of artificial light calibrator sources with ideal characteristics such as even frequency spacing, subresolution linewidth and uniform intensity across the spectral window of the spectrograph. Sources with these characteristics are the so called optical frequency combs, also known as astrocombs. Two main approaches have been followed to generate such reference sources, namely the Fabry-Perot etalon (passive device) and the laser frequency comb (active device). Both approaches are based on the property of optical resonators to sustain a multitude of longitudinal modes (resonances) in between the reflecting surfaces of the resonator. The allowed propagation modes shape the transmission spectrum of the resonator into a comb. The resonance frequencies of an achromatic resonator $v_{\mathrm{m}}$ are periodic and depend only on two parameters, the FSR (i.e., the periodicity of the resonances, FSR), and the offset frequency $v_{0}$ :

$$
v_{\mathrm{m}}=v_{0}+m \cdot \mathrm{FSR},
$$

$m$ being an integer number identifying the resonance. The FSR is the inverse of the round-trip time of light in the resonator, which for a cavity of length $L$ composed by two parallel mirrors filled with a medium of refractive index $n$ corresponds to

$$
\mathrm{FSR}=\frac{c}{2 n L} .
$$

Another important parameter of cavities is the linewidth, which can be expressed in terms of the finesse (FSR over the full width half maximum of the linewidth) (Hecht 2012).

The simplest implementation of the optical frequency comb source is a FabryPerot etalon with a vacuum filling and illuminated by a collimated broadband light source. Key for the accuracy of the comb line position is to control the spacing between the mirrors with nanometric precision over time. This is typically achieved by high-precision temperature control of the environment of the etalon (Wildi et al. 2010), or by active tuning of the mirror gap monitored by a reference laser (Gurevich et al. 2014). Fiber pigtailed, vacuum-spaced, planar dielectric mirrors were used for the Fabry-Perot calibrator of HARPS (Wildi et al. 2010) and reported a calibration stability comparable to the ThAr lamps on short $(<20 \mathrm{~cm} / \mathrm{s})$ as well as long observation periods $(<1 \mathrm{~m} / \mathrm{s})$ (Wildi et al. 2011). The authors reported that possible slight misalignments of the fiber-to-fiber imaging system may be responsible for the observed calibration drift over long periods. Fabry-Perot etalons consisting of a short single-mode fiber patch with spliced mirrors and single-mode pigtails were proposed as possible alternative to bulk optics etalons inherently insensitive to the alignment of the illumination source (Halverson et al. 2013; Gurevich et al. 2014). Differently from vacuum-spaced bulk-optics etalons, fiber Fabry-Perot (FFP) resonators suffer of dispersion (i.e., the comb is not exactly periodic) and are more sensitive to temperature variations, requiring a temperature stabilization at the fraction of $\mathrm{mK}$ level to keep the wavelength drift of the 
resonances below $1 \mathrm{~m} / \mathrm{s}$ (Halverson et al. 2013). Precision temperature tuning of the FFP by monitoring the gap with a Rubidium referenced laser allowed the stabilization of the FFP resonances below the $20 \mathrm{~cm} / \mathrm{s}$ level averaging over an integration time of only $30 \mathrm{~s}$ (Gurevich et al. 2014). A portable calibrator for low to mid resolution, near-infrared spectrographs was engineered starting from an integrated optics micro-ring resonator with drop off channel (Lee et al. 2012). The micro-ring resonator featured a FSR of $200 \mathrm{GHz}$ and included a resistive thermal device on its surface for accurate control of the temperature. A fibered amplified spontaneous emission broadband source was butt-coupled to the input channel of the microring resonator, so that a bright line spectrum was dropped off at the output channel. A line wavelength stability of the order of $1 \mathrm{pm}$ at $1550 \mathrm{~nm}$ was achieved over a period of $24 \mathrm{~h}$, which corresponds to a RV precision of about $200 \mathrm{~m} / \mathrm{s}$.

The advent of laser frequency comb technology as ultimate time/frequency standards (Udem et al. 2002) stimulated research on their application as accurate calibrators for high-resolution astronomical spectroscopy. Laser frequency combs are ultrashort pulse (20-300 fs) laser sources where modulated resonator losses enable the locking of the longitudinal modes and the generation of an exactly periodic frequency comb. The key difference to a standard ultrashort laser pulse is that frequency combs use an external nonlinear interferometer referenced to an atomic clock to control the drift of the offset frequency through a feedback on the cavity length. The advantage of this scheme over conventional Fabry-Perot calibrators is the higher brilliance of the spectral lines and the stability of their position which is set by the precision of the atomic clock $\left(\Delta v / v \sim 10^{-11}\right.$ for a standard Rubidium clock). Two types of femtosecond laser sources have been used so far for astrocombs: fiber laser and solid-state laser. The former has the advantage of being a self-aligned, turn-key system, but require usually long patches of gain fibers, reducing the free-spectral range to a few $100 \mathrm{MHz}$. This line separation is too small to be resolved even by the spectrograph with the highest resolution $(\Delta v \sim 1$ $\mathrm{GHz}$ ), so that the spectral lines are selected by several actively stabilized FabryPerot cavities with free spectral range of the order of 10-20 GHz. A prototype of a fiber based, infrared comb was installed at the German Vacuum Tower Solar Telescope and delivered a calibration precision at the $9 \mathrm{~m} / \mathrm{s} \mathrm{level}\left(\Delta v / v \sim 3 \cdot 10^{-8}\right)$. The known drifts of the spectrograph were responsible for the measured precision, which, though remarkable, was poorer than the theoretical precision of the comb (Steinmetz et al. 2008). An improved, frequency doubled version of the astrocomb was later installed at HARPS and was used to complete a new atlas of the solar spectrum reflected by the Moon with significantly improved accuracy (Molaro et al. 2013). In the same measurement campaign, the astrocomb demonstrated a shorttime repeatability of the HARPS calibration at the $2.5 \mathrm{~cm} / \mathrm{s}$ level $\left(\Delta v / v \sim 8 \cdot 10^{-11}\right)$ (Wilken et al. 2012). Radial velocity measurements of HD75289 were also taken both with ThAr lamp and astro-comb calibration which agreed within an accuracy of $2.5 \mathrm{~m} / \mathrm{s}$, most probably limited by the ThAr lamp accuracy (Wilken et al. 2012). Solid-state, mode-locked lasers offer more compact cavities which can have an FSR of a few $\mathrm{GHz}$, relaxing the requirement for the Fabry-Perot cavity required to select an astrocomb with tens of $\mathrm{GHz}$ spacing, but introducing more degrees of freedom 
for the periodic alignment of the laser cavity. A stabilized frequency-doubled Ti:Sapphire laser with a free-spectral range of $1 \mathrm{GHz}$ was used in combination with a Fabry-Perot etalon to obtain a $20-50 \mathrm{GHz}$-spaced astro-comb operating in the blue region of the visible spectrum $(\lambda=420 \mathrm{~nm})$ and tested at the TRES spectrograph at the Fred Lawrence Whipple Observatory (Benedick et al. 2010). The frequency comb allowed for an accurate calibration at the $1 \mathrm{~m} / \mathrm{s}$ level $\left(\Delta v / v \sim 3 \cdot 10^{-9}\right)$ of the spectrograph which showed drifts of a few $100 \mathrm{~m} / \mathrm{s}$ over days (Phillips et al. 2012). An upgraded version of the comb has been recently deployed at the HARPS-N spectrograph of the Telescopio Nazionale Galileo on La Palma and demonstrated a short time calibration uncertainty at the $2 \mathrm{~cm} / \mathrm{s}$ level (Glenday et al. 2015).

Besides the conventional architecture of laser frequency combs based on modelocked femtosecond laser sources, alternative schemes for the generation of trains of ultrafast laser pulses with $\mathrm{GHz}$ repetition rates have been explored. The aim of these schemes is to avoid the complexity and cost of the astrocombs based on modelocked lasers. A frequency comb with tunable FSR was designed and built by generating a train of near-infrared optical solitons in a nonlinear fiber seeded by the beating of two mutually detuned, continuous wave lasers (Zajnulina et al. 2015). Laboratory tests showed the possibility to obtain combs with sub-THz FSR, suitable for the calibration of low- to mid-resolution spectrographs. No stability characterization was carried out in the published work. More recently a nearinfrared astrocomb with $12 \mathrm{GHz}$ FSR was deployed at NASA IRTF telescope and Keck II (Yi et al. 2016). The source uses electrooptical modulators in phase and amplitude to generate a $12 \mathrm{GHz}$ train of 2 ps pulses from a continuous wave laser source, referenced to narrow molecular absorption lines of acetylene or cyanide. The radio frequency driving signal of the modulators is referenced to a Rubidium atomic clock, allowing an accurate control of the comb FSR at the $\Delta v / v \sim 10^{-11}$ level. After amplification in an erbium doped fiber, the pulse train spectrum was broadened in a highly nonlinear fiber allowing the comb to stretch from $1400 \mathrm{~nm}$ to $1700 \mathrm{~nm}$ wavelength. The intrinsic accuracy of the comb was measured in the lab to be below $60 \mathrm{~cm} / \mathrm{s}\left(\Delta v / v=3 \cdot 10^{-9}\right)$, which was degraded to an estimated level of $\sim 1.5 \mathrm{~m} / \mathrm{s}$ after taking into account the uncertainties related to the spectrograph operation.

Efforts to miniaturizing frequency combs for astronomical instrumentation have recently achieved a milestone by delivering a calibrator based on integrated microcombs at the Keck II telescope (Suh et al. 2019). Microcombs are frequency combs excited in a microring resonator by means of an optical nonlinear process known as four-wave-mixing. The resonator is pumped with a monochromatic laser at frequency $v_{0}$ tuned near the resonance frequency of the microring. The resonator stores a very strong optical field, which modifies the refractive index of the resonator and enables the split of two photons at $v_{0}$ in a signal and an idler photon following the principle of energy conservation:

$$
v_{ \pm}=v_{0} \pm \Delta v
$$

Each new line in the spectrum can be considered as a pump field, so that a cascade 
of equally spaced frequencies can be generated (Herr et al. 2012). In low dispersion resonators a wide comb can be generated and phase locked, generating a single optical pulse circulating in the resonator (Herr et al. 2014). Because of the small dimension of the microring resonators, the frequency spacing of the generated comb is in the range of a few $10 \mathrm{GHz}$, making them attractive for the calibration of astronomical spectrographs. In the reported work (Suh et al. 2019), a 70 nm-wide comb centered at a wavelength of $1550 \mathrm{~nm}$ was generated in a silicon microdisk resonator excited by a suspended fiber taper. The comb was referenced against a hydrogen cyanide gas cell (absorption line at $1559.9 \mathrm{~nm}$ ) with a precision better than $1 \mathrm{MHz}$, since the spectrum was not wide enough to apply the usual selfreferencing method. This provided a radial velocity precision of about $1 \mathrm{~m} / \mathrm{s}$. The comb was then extended to cover almost completely the H-band by passing the generated pulses into a patch of nonlinear fiber and projected into the NIRSPEC $R=25,000$ infrared spectrograph at the Keck II telescope. The drift of the spectrometer could be determined within a precision of a few $\mathrm{m} / \mathrm{s}$, mostly limited by the geometry of the spectrograph and detector. The study allowed to identify a roadmap for the upgrade of the spectrograph aimed at limiting its thermo-mechanical drifts and improving its resolving power by means of adaptive optics on the incoming stellar beam. Even tough the whole microcomb setup required about $1.3 \mathrm{~m}$ of a standard instrumental rack, there is a potential for integrating microdisks and pump sources within a chip of a few square centimeters footprint.

Wavefront sensing and adaptive optics Fiber bundles have been used as tools for guiding telescopes for many years, for example the fiber guiding mode using the FLAMES IFU at the VLT. This instrument uses four fiducial acquisition bundles (FACBs) to record positions of reference stars and then pass any offset to the instrument guiding system. With SM astrophotonics gaining popularity, accurate guiding and compensation for vibrations in the telescope system becoming more urgent. Dietrich et al. (2017) proposed using a MCF fed with 3D printed microlenses to compensate for tip-tilt vibrations, to increase coupling into SM fed instruments. This concept has been further developed by Hottinger et al. (2018), taking the MCF and replacing it with a fiber bundle for increased sensing and linearity. Outside astronomy there are also similar concepts, including using a photonic crystal fiber (PCF) for wavefront sensing (Dos Santos et al. 2015). Another avenue being actively pursued is using a PL at the focal plane to monitor the PSF of the telescope. As the light separates from the MM core into many SMs any irregularities in the wavefront result in different modes being excited. By monitoring the output of the PL (Corrigan et al. 2016) were able to detect tip and tilt in the PSF using a simulated 9 mode PL. Recently, Norris et al. (2020) have extended this concept, showing that they can detect higher order modes using a 19 core PL, opening up the possibility of using a PL for full photonic wavefront sensing for applications such as exoplanet science.

In addition to using astrophotonics for wavefront sensing, using active waveguides instead of a deformable mirror is also under investigation. Miller (2013) proposed that on-chip cascaded Mach-Zehnder interferometers in conjunction with a PL could be used as an AO system (see Fig. 14). This would take an input PSF and use micro phase shifters on the chip to constructively interfere the 


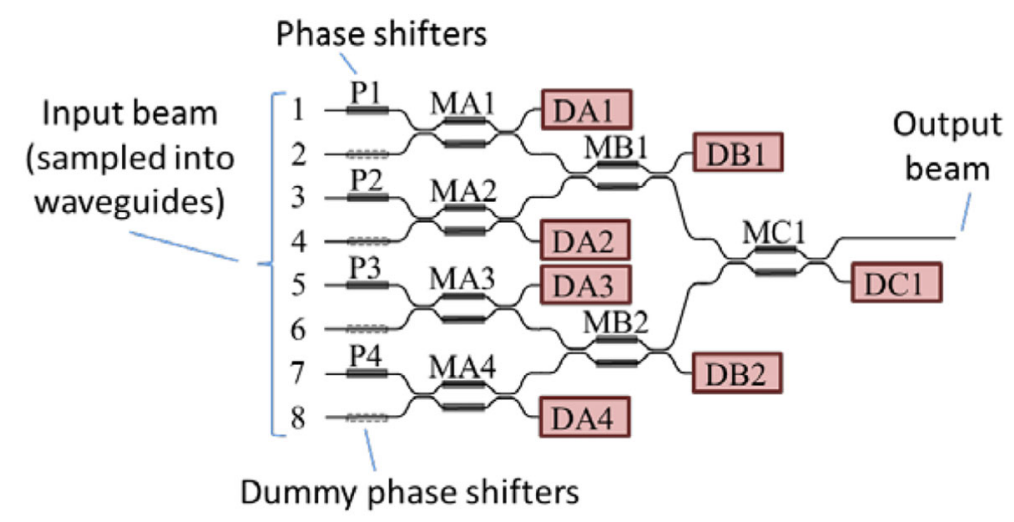

Fig. 14 One approach for coupling an arbitrary input beam to a single output beam, so-called Adaptive Optics (AO) on a chip. In the figure, P1-P4 are controllable phase shifters, MA1-MC1 are controllable MZ interferometers, and DA1-DC1 are detectors used to give the signals for feedback loops. The dummy phase shifters are optional and could be included for equality of path lengths and/or loss. Image reproduced with permission from Miller (2013), copyright by OSA

individual modes. Sensed correctly, this would either enhance, or potentially replace an AO system, in a fully integrated chip.

\subsection{Detection enhancement through frequency conversion}

A remarkable research topic bearing potentially important consequences for the future of infrared astronomy is related to the nonlinear optical conversion of light frequencies in periodically poled lithium niobate (PPLN) crystals (Albota and Wong 2004; Langrock et al. 2005). It is well known that infrared detectors have quantum efficiencies well below their visible counterparts, especially in the MIR bands. Moreover, as we have seen, photonic components for the MIR are currently still under development. A solution to these problems could come from nonlinear frequency conversion.

Nonlinear frequency conversion is a process exploiting the nonlinear polarization response of non-centrosymmetric crystals. In such crystals the polarization vector has a term depending on the square of the electric field (nonlinear polarization). If the driving electric field has two frequency components $\omega_{1}$ and $\omega_{2}$, then the nonlinear polarization will contain a term oscillating at the sum-frequency $\omega_{3}=$ $\omega_{1}+\omega_{2}$ which gives rise to a radiating field (frequency up-conversion process). The sum frequency field can build up coherently as it propagates in the medium provided the phase-matching condition is fulfilled, i.e., the propagation vector at frequency $\omega_{3}$ should equal the sum of the wavevectors of the two initial fields: $\mathbf{k}\left(\omega_{3}\right)=\mathbf{k}\left(\omega_{1}\right)+\mathbf{k}\left(\omega_{2}\right)$. Usually phase matching is achieved by exploiting the birefringence of quadratic nonlinear materials, but in this case the phase matching direction may not coincide with the direction of higher nonlinear coefficient. In periodically poled materials, phase matching is achieved by inverting periodically 
the orientation of the crystal, so that the phase matching direction coincides with the direction where the nonlinear coefficient of the crystal is maximum.

The first application of nonlinear frequency conversion to enhance the detection quantum efficiency of infrared photons was reported in Albota and Wong (2004). In the experiments, a $4 \mathrm{~cm}$-long PPLN crystal was operated within a singly resonant ring cavity, pumped with a $400 \mathrm{~mW}$ Nd:YAG laser at a wavelength of $1064 \mathrm{~nm}$. Photons at the wavelength of $1.55 \mu \mathrm{m}$ were converted to visible light $(\lambda=631 \mathrm{~nm})$ with an estimated efficiency of $90 \%$ (the ratio of upconverted photons to the input in infrared photons). The setup for the wavelength conversion was later miniaturized by Langrock et al. (2005), who used a waveguide written in the PPLN crystal to achieve high conversion efficiency with a pump power of a few tens of $\mathrm{mW}$. The scheme could be used to convert either photons at $\lambda=1.32 \mu \mathrm{m}$ or $\lambda=1.55 \mu \mathrm{m}$ to photons at $\lambda=713 \mathrm{~nm}$, to exploit the maximal quantum efficiency of silicon, single-photon detectors. In the experiments, an overall quantum detection efficiency of $40 \%$ was reported.

While these experiments were mainly motivated by quantum optics applications, the first use of optical frequency conversion for astronomy was proposed by Brustlein et al. (2008). The seminal paper investigated the coherence properties of unconverted light in PPLN waveguides. To this end, a fiber interferometer was built, featuring a wavelength converter in each arm (see Fig. 15).

By combining the up-converted beams, interference fringes were recorded with a visibility corresponding to the independent measurements taken by combining the original infrared signals. The experiments showed unequivocally that the coherence properties of light are preserved by the up-conversion scheme. Further confirmation came later on with an experiment performed with a 3-channel fiber interferometer which showed that also the closure phases are preserved by the up-conversion scheme (Ceus et al. 2011).

The frequency conversion scheme was later tested on sky (Ceus et al. 2012) by connecting one arm of the frequency converter to a C8 Celestron telescope used for the OHANA-Iki project (Baril et al. 2010). The setup allowed converting a bandwidth of $3 \mathrm{~nm}$ centered at $1544 \mathrm{~nm}$ ( $\mathrm{H}$ band) in a $4 \mathrm{~cm}$-long PPLN waveguide. Even with this narrow conversion bandwidth, a flux of a few tens of photons per second was recorded for observations of Betelgeuse, Antares and Pollux. The calculated overall detection quantum efficiency was estimated to be $1.75 \cdot 10^{-6}$, but this included a particularly low coupling efficiency to the single-mode fiber $\left(3.5 \cdot 10^{-3}\right)$ and the insertion losses of the various optical elements (filters, lenses, collimators). Theoretically (Shaklan and Roddier 1988), an increase of the coupling efficiency from telescope to fiber by a factor 100 can be expected, while the use of integrated optics instead of bulk optics relay and higher laser pump powers could increase further the efficiency of the photon conversion stage (overall conversion efficiency $1 \%$ ).

Despite the low overall quantum detection efficiency of the first on sky test, the result of Ceus et al. (2012) opens a large range of perspectives for the application of nonlinear optics to astronomy. It is easy to think about potential applications such as conversion of MIR light into telecom wavelengths to exploit the availability of 


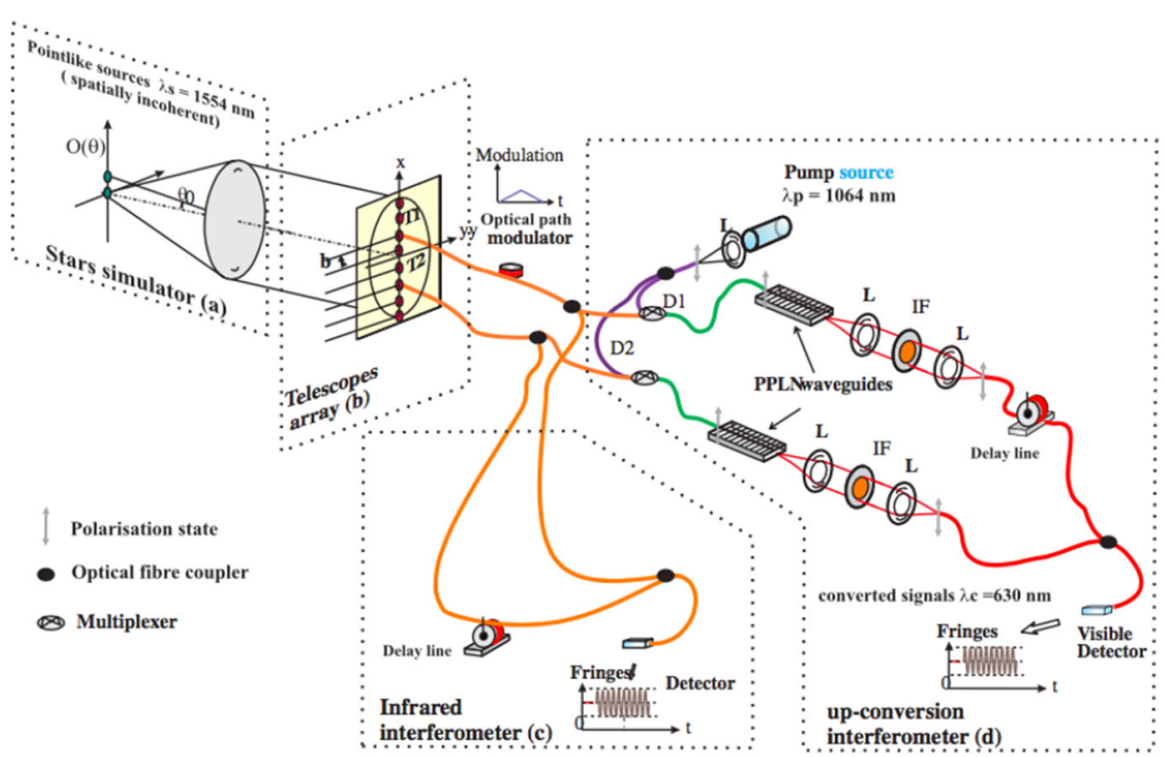

Fig. 15 Scheme of the experimental layout of a fiber interferometer with optical frequency conversion stage. An infrared light source consisting of two point sources is collimated and sampled by two fiber couplers which could move across the collimated beam, thus simulating an astronomical interferometer observing a binary star. The sampled light is carried by the fibers to a coupler which injects the pump beam necessary to generate the optical sum frequency in the periodically poled lithium niobate waveguides (PPNL). The generated visible light from each PPNL crystal is then coupled into a fiber suitable for visible light and mixed inside a $2 \times 2$ coupler before being detected. A parallel infrared interferometer is built for reference purposes. Image reproduced with permission from Brustlein et al. (2008), copyright by APS

photonic components for signal transport and processing over large distances. This is indeed the basic concept that the project ALOHA aims to test at the CHARA interferometric array (Lehmann et al. 2018b). The project aims at enabling MIR interferometric observations by converting MIR photons to the telecom wavelengths by means of a PPLN waveguide and transferring the up-converted light to the beam combiner using hectometric single-mode optical fibers (Lehmann et al. 2019). As the efficient conversion of photons requires long lithium niobate waveguides (2-4 $\mathrm{cm}$ ), the conversion bandwidth is small and only narrow lines of the MIR spectrum can be explored. A possibility to extend the bandwidth of the the frequency conversion is to use several non-redundant phase-modulated pump sources at slightly different wavelengths which achieve phase-matching at different portions of the MIR spectrum (Lehmann et al. 2018a). The phase modulation of the different pump sources allows disentangling the MIR spectral channels by a simple Fouriertransformation of the interferometric signal of both arms of the interferometer. The initial results of this project are very promising, however the conversion efficiency of the PPLN conversion stage requires improvements (e.g., longer PPLN, higher pump power, reduction of insertion losses), as the achieved overall conversion efficiency is in the order of $0.4 \%$ (Lehmann et al. 2018a). 


\section{Conclusion and future}

In this paper, we have discussed how state-of-the-art optical technologies, i.e., photonics, are slowly being incorporated into the design and realization of new astronomical instrumentation with enhanced performance and which could exploit the light collection parameters of current and forthcoming large or extremely large telescopes. This process of testing advanced optical technologies to investigate the sky can be seen as a continuation of a scientifically fruitful tradition initiated by Galileo's attempt to point his telescope to the Moon and Jupiter. As discussed in Sect. 2, a number of important scientific cases would benefit greatly from widespread incorporation of photonic technologies thanks to their intrinsic small footprint, multiplexing capability and potentially superior performance as compared to bulk optics, provided the PSF of the telescope is suitably controlled.

As we have seen, the small footprint offered by integrated optical components is a major asset for astrophotonic instrumentation. This aspect has been already exploited to deliver extremely compact beam combiners for optical astronomical interferometry, which offer enhanced stability to climatic and mechanical perturbations of the instrument (see Sect. 4.3). Additionally, several groups are trying to develop miniaturized integrated photonic spectrographs, (Sect. 4.1), with many replicas of the miniaturized devices connected to the ends of a photonic lantern (Cvetojevic et al. 2012) or a single miniaturized device is linked to an AO-corrected telescope (Jovanovic et al. 2017a). Miniaturization can also bring tremendous benefits to space borne instrumentation, saving precious fuel or enabling more experiments on the same space probe. In this context, we mention the flight in 2017 of an integrated photonic spectrometer (Betters et al. 2012) on the Australian nanosatellite i-INSPIRE 2, and the attempt to launch in early 2018 an integrated optics interferometric beam combiner with the French PicSat (Lacour et al. 2014). More experiments will certainly follow in the future and demonstrate the full potential of astrophotonics technologies for space astronomy.

Related to miniaturization is also the opportunity to integrate in the instrument many small copies of optical devices delivering or processing the light signal from the sky. Under this aspect, the introduction of optical fibers in spectroscopy revolutionized astronomy, allowing to multiply by up to three orders of magnitude the productivity of spectrographs (Sect. 4.2) or to enable spatially and spectrally resolved images of distant galaxies (Sect. 4.2). This trend will certainly continue in the future as planned large scale MOSs (e.g., 4MOST, or ELT/MOSAIC) will see the light and new astrophotonic functionalities may be added (e.g., multiple PIMMS; Bland-Hawthorn and Horton 2006).

Enhanced performance is also a key element of astrophotonic instrumentation, which may develop fully in the coming years. As we mentioned in Sect. 4.3, the superior performance of integrated optics beam combiners for optical interferometry has been already demonstrated and employed in instruments such as GRAVITY, which promises to revolutionize our knowledge on the supermassive black-hole nested at the center of our Galaxy (GRAVITY Collaboration et al. 2017). Extension of the concept to MIR wavelengths is rapidly progressing (Diener et al. 2017; 
Kenchington Goldsmith et al. 2017b; Labadie et al. 2018), as is the increase of the number of combined telescopes at the yet unexplored $\mathbf{J}$ spectral band (Pedretti et al. 2018). As we have seen in Sect. 4.1, photonics is the only viable existing option to enable the suppression of telluric $\mathrm{OH}$ emission lines before the spectrograph, a key functionality for spectroscopy of galaxies with redshifts between 2 and 3 (the cosmic noon). While proof-of-principle experiments have been already carried out with GNOSIS, the project PRAXIS has moved a step forward from technology towards science demonstrator. Active photonics is also becoming more and more an essential tool of modern astronomy. Laser Guide Stars (Sect. 4.5) will be standard equipment in large and extremely large telescopes required to extend the use of AO to fainter objects, while laser frequency combs (Sect. 4.5) promise to become the ultimate calibrators for mid- and high-resolution spectroscopy. Laser light sources are becoming also common in space exploration. For instance, the ChemCam of NASA's Curiosity Mars Rover features a powerful nanosecond pulse infrared laser to perform laser-induced breakdown spectroscopy (LIBS) of distant rocks. Light emitted by the laser plasma is delivered via optical fiber from the collection optics on a robotic arm to the spectrometer hosted in the chassis of the rover. In the future, lasers on board of deep-space probes could enable extremely accurate ranging to push our knowledge on planetary dynamics to unprecedented precision (Smith et al. 2006), while large datasets could be beamed optically to Earth at a rate of several hundred Mbit/s (Bland-Hawthorn et al. 2002; Sodnik et al. 2014; Robinson et al. 2014) exploiting the high brilliance of laser sources.

We believe these three features will be the cornerstones of future applications of photonic technologies to astronomical instrumentation. While the choice between astrophotonic and conventional solutions has to be evaluated for each instrumental project, at present there are three main challenges for the deployment of the potential of photonic technologies in astronomy.

One key issue affecting mainly single-mode astrophotonic components is the low throughput, which is mainly due to the mismatch in the modal content of uncorrected starlight and the supported modes in the photonic component (see Sect. 3). This problem is particularly severe for large telescope apertures and short optical wavelengths. Besides using photonic lanterns to sort multimode light into several single-mode outputs, the definitive solution to this problem is matching the single-mode components to a telescope equipped with AO. While diffractionlimited beams are already possible at infrared wavelengths on 8-meter class telescopes (Dekany et al. 2013; Jovanovic et al. 2014), recent advances allow the achievement of moderate Strehl ratios in the visible band at 6-meter-class telescopes (Pedichini et al. 2017). At the same time, the development of micro-electromechanical systems has consistently reduced the prices of high-order deformable mirrors, making AO more affordable. We expect that the increasing number of telescopes equipped with moderate to extreme AO systems will contribute to increase the competitiveness of single-mode astrophotonic instrumentation such as PIMMS, while bringing to fruition the decade-long development of phase mask coronagraphs (Sect. 4.4).

A second aspect hindering the use of astrophotonic components is the relatively narrow spectral coverage of existing manufacturing technologies. Most of the 
photonic components have been optimized for the digital telecommunication market, which operates in a narrow infrared band (approximatively corresponding to the shorter half of the astronomical H-band), where silica is most transparent. The availability of commercial components outside this range is scarce and often not compliant with the requirements of astronomical use, e.g., they feature higher insertion or scattering losses compared to telecom band equivalents. Clearly, astronomers will need to rely on existing manufacturing technologies, as the optimization of photonic production processes is beyond the possibilities of the community. However, growing markets, such as biophotonics and security, currently drive photonic applications in the visible and MIR bands, which may deliver the required platforms for the development of astrophotonic components. As we have seen in Sect. 4.3, advances in manufacturing technologies for MIR photonics are behind the recent progress towards MIR integrated optics beam combiners for stellar interferometry.

A third aspect is the achievable dynamical range of integrated optical devices. As mentioned, these have mostly been developed for digital telecommunications where modulation contrasts of $20 \mathrm{~dB}$ (1:100) or less are usually sufficient to reduce bit error rates to negligible values. With these specifications, stray light originating from modal mismatches or scattering in waveguides does not affect substantially the performance of the devices. In contrast, several astronomical science cases (such as high-precision spectroscopy, coronagraphy or nulling interferometry) require contrasts exceeding $30 \mathrm{~dB}$. At these levels the already mentioned sources of stray light may turn out to be unacceptable and pose a challenge for the development of astronomy-grade integrated optics.

We put forward that dealing with these challenges will be at the focal point of new astrophotonic developments in the years to come, which will certainly witness new fascinating discoveries in astronomy enabled by increasingly sophisticated instrumentation, where state-of-the-art photonics will play a crucial role.

Acknowledgements The authors acknowledge financial support by the DFG through the Grant 326946494 "NAIR - Innovative Astronomische Instrumentierung mittels photonischer Reformatierer". SM would like to thank his family for allowing him to devote many evenings to the writing of the manuscript. RJH would like to thank Rosalie McGurk for her useful input regarding the current questions important to astronomy.

Funding Open Access funding enabled and organized by Projekt DEAL.

Open Access This article is licensed under a Creative Commons Attribution 4.0 International License, which permits use, sharing, adaptation, distribution and reproduction in any medium or format, as long as you give appropriate credit to the original author(s) and the source, provide a link to the Creative Commons licence, and indicate if changes were made. The images or other third party material in this article are included in the article's Creative Commons licence, unless indicated otherwise in a credit line to the material. If material is not included in the article's Creative Commons licence and your intended use is not permitted by statutory regulation or exceeds the permitted use, you will need to obtain permission directly from the copyright holder. To view a copy of this licence, visit http:// creativecommons.org/licenses/by/4.0/. 


\section{References}

Akerlof C, Amrose S, Balsano R, Bloch J, Casperson D, Fletcher S, Gisler G, Hills J, Kehoe R, Lee B, Marshall S, McKay T, Pawl A, Schaefer J, Szymanski J, Wren J (2000) ROTSE All-Sky Surveys for Variable Stars. I. Test Fields. AJ 119(4):1901-1913. https://doi.org/10.1086/301321. arXiv:astro-ph/ 0001388 [astro-ph]

Albota MA, Wong FNC (2004) Efficient single-photon counting at $1.55 \mu \mathrm{m}$ by means of frequency upconversion. Opt Lett 29:1449-1451. https://doi.org/10.1364/OL.29.001449

Alcock C, Akerlof CW, Allsman RA, Axelrod TS, Bennett DP, Chan S, Cook KH, Freeman KC, Griest K, Marshall SL, Park HS, Perlmutter S, Peterson BA, Pratt MR, Quinn PJ, Rodgers AW, Stubbs CW, Sutherland W (1993) Possible gravitational microlensing of a star in the Large Magellanic Cloud. Nature 365:621-623. https://doi.org/10.1038/365621a0. arXiv:astro-ph/9309052

Allington-Smith J (2006) Basic principles of integral field spectroscopy. New A Rev 50(4-5):244-251. https://doi.org/10.1016/j.newar.2006.02.024

Allington-Smith J, Murray G, Content R, Dodsworth G, Davies R, Miller BW, Jorgensen I, Hook I, Crampton D, Murowinski R (2002) Integral field spectroscopy with the Gemini multiobject spectrograph. I. Design, construction, and testing. PASP 114(798):892-912. https://doi.org/10.1086/ 341712

Angel JRP, Woolf NJ (1997) An imaging nulling interferometer to study extrasolar planets. ApJ 475:373-379. https://doi.org/10.1086/303529

Anglada-Escudé G, Amado PJ, Barnes J, Berdiñas ZM, Butler RP, Coleman GAL, de La Cueva I, Dreizler S, Endl M, Giesers B, Jeffers SV, Jenkins JS, Jones HRA, Kiraga M, Kürster M, LópezGonzález MJ, Marvin CJ, Morales N, Morin J, Nelson RP, Ortiz JL, Ofir A, Paardekooper SJ, Reiners A, Rodríguez E, Rodríguez-López C, Sarmiento LF, Strachan JP, Tsapras Y, Tuomi M, Zechmeister M (2016) A terrestrial planet candidate in a temperate orbit around Proxima Centauri. Nature 536:437-440. https://doi.org/10.1038/nature19106. arXiv:1609.03449 [astro-ph.EP]

Arsenault R, Madec PY, Paufique J, Penna PL, Ströbele S, Vernet E, Pirard JF, Hackenberg W, Kuntschner H, Jochum L, Kolb J, Muller N, Louarn ML, Amico P, Hubin N, Lizon JL, Ridings R, Abad J, Fischer G, Heinz V, Kiekebusch M, Argomedo J, Conzelmann R, Tordo S, Donaldson R, Soenke C, Duhoux P, Fedrigo E, Delabre B, Jost A, Duchateau M, Downing M, Moreno J, Dorn R, Manescau A, Calia DB, Quattri M, Dupuy C, Guidolin I, Comin M, Guzman R, Buzzoni B, Quentin J, Lewis S, Jolley P, Kraus M, Pfrommer T, Biasi R, Gallieni D, Bechet C, Stuik R (2012) ESO adaptive optics facility progress report. In: Ellerbroek BL, Marchetti E, Véra JP (eds) Adaptive Optics Systems III. Proceedings of SPIE, vol 8447. SPIE, p 84470J. https://doi.org/10.1117/12. 926074

Artigau É, Kouach D, Donati JF, Doyon R, Delfosse X, Baratchart S, Lacombe M, Moutou C, Rabou P, Parès LP, Micheau Y, Thibault S, Reshetov VA, Dubois B, Hernandez O, Vallée P, Wang SY, Dolon F, Pepe FA, Bouchy F, Striebig N, Hénault F, Loop D, Saddlemyer L, Barrick G, Vermeulen T, Dupieux M, Hébrard G, Boisse I, Martioli E, Alencar SHP, do Nascimento JD, Figueira P (2014) SPIRou: the near-infrared spectropolarimeter/high-precision velocimeter for the Canada-FranceHawaii telescope. In: McLean IS, Ramsay SK, Takami H (eds) Ground-based and Airborne Instrumentation for Astronomy V. Proc. SPIE. , vol 9147. SPIE, p 914715. https://doi.org/10.1117/ 12.2055663. arXiv:1406.6992 [astro-ph.IM]

Avila G, Guirao C, Baader T (2012) High efficiency inexpensive 2-slices image slicers. In: McLean IS, Ramsay SK, Takami H (eds) Ground-based and Airborne Instrumentation for Astronomy IV. Proc. SPIE., vol 8446. SPIE, p 84469M. https://doi.org/10.1117/12.927448

Bacon R, Copin Y, Monnet G, Miller BW, Allington-Smith JR, Bureau M, Marcella Carollo C, Davies RL, Emsellem E, Kuntschner H et al (2001) The SAURON project - I. The panoramic integral-field spectrograph. MNRAS 326(1):23-35. https://doi.org/10.1046/j.1365-8711.2001.04612.x

Bacon R, Accardo M, Adjali L, Anwand H, Bauer S, Biswas I, Blaizot J, Boudon D, Brau-Nogue S, Brinchmann J, et al (2010) The MUSE second-generation VLT instrument. In: McLean IS, Ramsay SK, Takami H (eds) Ground-based and Airborne Instrumentation for Astronomy III. Proceedings of SPIE, vol 7735. SPIE, p 773508. https://doi.org/10.1117/12.856027

Bailyn C, Clemens C, Johnson J, Joseph R, Kawaler S, Salzer J, Thornley M, Weintraub D (2007) Renewing small telescopes for astronomical research (final report). Tech. rep., NOAO. URL https:// www.noao.edu/system/restar/ 
Baldwin JE, Haniff CA, Mackay CD, Warner PJ (1986) Closure phase in high-resolution optical imaging. Nature 320:595-597. https://doi.org/10.1038/320595a0

Bally J, O’Dell CR, McCaughrean MJ (2000) Disks, microjets, windblown bubbles, and outflows in the Orion Nebula. AJ 119:2919-2959. https://doi.org/10.1086/301385

Baranne A, Queloz D, Mayor M, Adrianzyk G, Knispel G, Kohler D, Lacroix D, Meunier JP, Rimbaud G, Vin A (1996) ELODIE: a spectrograph for accurate radial velocity measurements. A\&AS 119:373-390

Baril M, Lai O, Zahariade G, Bouchacourt F, Perrin G, Fedou P, Woillez J (2010) 'OHANA-Iki: A testbed for the 'OHANA beam combiner and delay line at CFHT. In: Danchi WC, Delplancke F, Rajagopal JK (eds) Optical and infrared interferometry II. Proceedings of SPIE, vol 7734. SPIE, p 77342C. https://doi.org/10.1117/12.856219

Baudrand J, Walker GAH (2001) Modal noise in high-resolution, fiber-fed spectra: a study and simple cure. PASP 113(785):851-858. https://doi.org/10.1086/322143

Bely P (2003) The design and construction of large optical telescopes. Astronomy and astrophysics library, Springer, New York. https://doi.org/10.1007/b97612

Benedick AJ, Chang G, Birge JR, Chen LJ, Glenday AG, Li CH, Phillips DF, Szentgyorgyi A, Korzennik S, Furesz G, Walsworth RL, Kärtner FX (2010) Visible wavelength astro-comb. Opt Express 18:19175. https://doi.org/10.1364/OE.18.019175

Benisty M, Berger JP, Jocou L, Labeye P, Malbet F, Perraut K, Kern P (2009) An integrated optics beam combiner for the second generation VLTI instruments. A\&A 498:601-613

Benisty M, Juhasz A, Boccaletti A, Avenhaus H, Milli J, Thalmann C, Dominik C, Pinilla P, Buenzli E, Pohl A, Beuzit JL, Birnstiel T, de Boer J, Bonnefoy M, Chauvin G, Christiaens V, Garufi A, Grady C, Henning T, Huelamo N, Isella A, Langlois M, Ménard F, Mouillet D, Olofsson J, Pantin E, Pinte C, Pueyo L (2015) Asymmetric features in the protoplanetary disk MWC 758. A\&A 578:L6. https:// doi.org/10.1051/0004-6361/201526011. arXiv:1505.05325 [astro-ph.SR]

Berger JP, Rousselet-Perraut K, Kern P, Malbet F, Schanen-Duport I, Reynaud F, Hagenauer P, Benech P (1999) Integrated optics for astronomical interferometry - II. First laboratory white-light interferograms. A\&AS 139:173-177. https://doi.org/10.1051/aas:1999504

Berger JP, Benech P, Schanen-Duport I, Maury G, Malbet F, Reynaud F (2000) Combining up to eight telescope beams in a single chip. In: Léna P, Quirrenbach A (eds) Interferometry in optical astronomy. Proceedings of SPIE, vol 4006. SPIE, pp 986-995. https://doi.org/10.1117/12.390177

Berger JP, Hagenauer P, Kern P, Perraut K, Malbet F, Schanen I, Severi M, Millan-Gabet R, Traub W (2001) Integrated optics for astronomical interferometry. IV. First measurements on stars. A\&A 376:L31-L34. https://doi.org/10.1051/0004-6361:20011035

Berger JP, Hagenauer P, Kern P, Perraut K, Malbet F, Gluck S, Lagny L, Schanen I, Laurent E, Delboulbe A, Magnard Y, Tatulli E, Traub W, Carleton N, Millan-Gabet R, Monnier JD, Pedretti E (2003) An integrated-optics 3-way beam combiner for IOTA. In: Traub WA (ed) Interferometry for Optical Astronomy II. Proceedings of SPIE, vol 4838. SPIE, pp 1099-1106. https://doi.org/10.1117/12. 457983

Berry M (1984) Quantal phase factors accompanying adiabatic changes. Proc R Soc Lond A Math Phys Eng Sci 392(1802):45-57. https://doi.org/10.1098/rspa.1984.0023

Besse PA, Bachmann M, Melchior H, Soldano LB, Smit MK (1994) Optical bandwidth and fabrication tolerances of multimode interference couplers. J Lightwave Technol 12:1004-1009. https://doi.org/ $10.1109 / 50.296191$

Betters C, Leon-Saval S, Bland-Hawthorn J (2012) NanoSpec: A diffraction limited micro-spectrograph for pico- and nano-satellites. In: Clampin MC, Fazio GG, MacEwen HA, Oschmann Jr JM (eds) Space telescopes and instrumentation 2012: optical, infrared, and millimeter wave. Proceedings of SPIE, vol 8442. SPIE, p 84424B. https://doi.org/10.1117/12.925819

Bezuhanov K, Dreischuh A, Paulus GG, Schätzel MG, Walther H (2004) Vortices in femtosecond laser fields. Opt Lett 29:1942-1944. https://doi.org/10.1364/OL.29.001942

Bienfang JC, Denman CA, Grime BW, Hillman PD, Moore GT, Telle JM (2003) 20 W of continuouswave sodium $D_{2}$ resonance radiation from sum-frequency generation with injection-locked lasers. Opt Lett 28:2219. https://doi.org/10.1364/OL.28.002219

Birks TA, Knight JC, Russell PSJ (1997) Endlessly single-modephotonic crystal fiber. Opt Lett 22(13):961-963. https://doi.org/10.1364/OL.22.000961

Birks TA, Mangan BJ, Díez A, Cruz JL, Murphy DF (2012) "Photonic lantern" spectral filters in multicore Fiber. Opt Express 20:13996. https://doi.org/10.1364/OE.20.013996 
Birks TA, Gris-Sánchez I, Yerolatsitis S, Leon-Saval SG, Thomson RR (2015) The photonic lantern. Adv Opt Photon 7(2):107-167. https://doi.org/10.1364/AOP.7.000107

Blais-Ouellette S, Artigau É, Havermeyer F, Matthews K, Moser C, Psaltis D, Steckman GJ (2004) Multinotch holographic filters for atmospheric lines suppression. In: Atad-Ettedgui E, Dierickx P (eds) Optical fabrication, metrology, and material advancements for telescopes. Proceedings of SPIE, vol 5494, pp 554-561. https://doi.org/10.1117/12.552116

Blake C, Johnson J, Plavchan P, Sliski D, Wittenmyer RA, Eastman JD, Barnes S (2015) MINERVARed: a census of planets orbiting the nearest low-mass stars to the sun. In: American Astronomical Society Meeting Abstracts \#225. vol 225, p 257.32

Bland-Hawthorn J, Horton A (2006) Instruments without optics: an integrated photonic spectrograph. In: McLean IS, Iye M (eds) Ground-based and airborne instrumentation for astronomy. Proceedings of SPIE, vol 6269. SPIE, p 62690N. https://doi.org/10.1117/12.670931

Bland-Hawthorn J, Kern P (2009) Astrophotonics: a new era for astronomical instruments. Opt Express 17:1880-1884. https://doi.org/10.1364/OE.17.001880. arXiv:0902.0415 [astro-ph.IM]

Bland-Hawthorn J, Leon-Saval SG (2017) Astrophotonics: molding the flow of light in astronomical instruments. Opt Express 25:15549. https://doi.org/10.1364/OE.25.015549. arXiv:1706.05132 [astro-ph.IM]

Bland-Hawthorn J, Harwit A, Harwit M (2002) Laser telemetry from space. Science. https://doi.org/10. 1126/science. 1074113

Bland-Hawthorn J, Englund M, Edvell G (2004) New approach to atmospheric OH suppression using an aperiodic fibre Bragg grating. Opt Express 12(24):5902-5909. https://doi.org/10.1364/OPEX.12. 005902

Bland-Hawthorn J, Lawrence J, Robertson G, Campbell S, Pope B, Betters C, Leon-Saval S, Birks T, Haynes R, Cvetojevic N, et al. (2010) PIMMS: photonic integrated multimode microspectrograph. In: McLean IS, Ramsay SK, Takami H (eds) Ground-based and airborne instrumentation for astronomy III. Proceedings of SPIE, vol 7735. SPIE. https://doi.org/10.1117/12.856347

Bland-Hawthorn J, Ellis SC, Leon-Saval SG, Haynes R, Roth MM, Löhmannsröben HG, Horton AJ, Cuby JG, Birks TA, Lawrence JS et al (2011) A complex multi-notch astronomical filter to suppress the bright infrared sky. Nat Commun 2:581. https://doi.org/10.1038/ncomms1584

Boccaletti A, Riaud P, Baudoz P, Baudrand J, Rouan D, Gratadour D, Lacombe F, Lagrange AM (2004) the four-quadrant phase mask coronagraph. IV. First light at the very large telescope. PASP 116:1061-1071. https://doi.org/10.1086/425735

Boccaletti A, Thalmann C, Lagrange AM, Janson M, Augereau JC, Schneider G, Milli J, Grady C, Debes J, Langlois M, Mouillet D, Henning T, Dominik C, Maire AL, Beuzit JL, Carson J, Dohlen K, Engler N, Feldt M, Fusco T, Ginski C, Girard JH, Hines D, Kasper M, Mawet D, Ménard F, Meyer MR, Moutou C, Olofsson J, Rodigas T, Sauvage JF, Schlieder J, Schmid HM, Turatto M, Udry S, Vakili F, Vigan A, Wahhaj Z, Wisniewski J (2015) Fast-moving features in the debris disk around AU Microscopii. Nature 526:230-232. https://doi.org/10.1038/nature15705

Bonaccini D, Hackenberg W, Cullum M, Brunetto E, Ott T, Quattri M, Allaert E, Dimmler M, Tarenghi M, Kersteren AV, DiChirico C, Buzzoni B, Gray P, Tamai R, Tapia M (2002) ESO VLT laser guide star facility. In: Tyson RK, Bonaccini D, Roggemann MC (eds) Adaptive Optics Systems and Technology II. Proceedings of SPIE, vol 4495. SPIE. https://doi.org/10.1117/12.454802

Bonnefoy M, Zurlo A, Baudino JL, Lucas P, Mesa D, Maire AL, Vigan A, Galicher R, Homeier D, Marocco F, Gratton R, Chauvin G, Allard F, Desidera S, Kasper M, Moutou C, Lagrange AM, Antichi J, Baruffolo A, Baudrand J, Beuzit JL, Boccaletti A, Cantalloube F, Carbillet M, Charton J, Claudi RU, Costille A, Dohlen K, Dominik C, Fantinel D, Feautrier P, Feldt M, Fusco T, Gigan P, Girard JH, Gluck L, Gry C, Henning T, Janson M, Langlois M, Madec F, Magnard Y, Maurel D, Mawet D, Meyer MR, Milli J, Moeller-Nilsson O, Mouillet D, Pavlov A, Perret D, Pujet P, Quanz SP, Rochat S, Rousset G, Roux A, Salasnich B, Salter G, Sauvage JF, Schmid HM, Sevin A, Soenke C, Stadler E, Turatto M, Udry S, Vakili F, Wahhaj Z, Wildi F (2016) First light of the VLT planet finder SPHERE. IV. Physical and chemical properties of the planets around HR8799. Astr Astroph 587:A58. https://doi.org/10.1051/0004-6361/201526906. arXiv:1511.04082 [astro-ph.EP]

Born M, Wolf E (1997) Principles of optics. Cambridge University Press. https://doi.org/10.1017/ 9781108769914

Bouchy F, Díaz RF, Hébrard G, Arnold L, Boisse I, Delfosse X, Perruchot S, Santerne A (2013) SOPHIE+: first results of an octagonal-section fiber for high-precision radial velocity measurements. A\&A 549:A49. https://doi.org/10.1051/0004-6361/201219979 
Bouquin JBL, Berger JP, Lazareff B, Zins G, Haguenauer P, Jocou L, Kern P, Millan-Gabet R, Traub W, Absil O, Augereau JC, Benisty M, Blind N, Bonfils X, Bourget P, Delboulbe A, Feautrier P, Germain M, Gitton P, Gillier D, Kiekebusch M, Kluska J, Knudstrup J, Labeye P, Lizon JL, Monin JL, Magnard Y, Malbet F, Maurel D, Ménard F, Micallef M, Michaud L, Montagnier G, Morel S, Moulin T, Perraut K, Popovic D, Rabou P, Rochat S, Rojas C, Roussel F, Roux A, Stadler E, Stefl S, Tatulli E, Ventura N, (2011) PIONIER: a 4-telescope visitor instrument at VLTI. A\&A 535:A67.

Bowler BP, Liu MC, Dupuy TJ, Cushing MC (2010) Near-infrared spectroscopy of the extrasolar planet HR 8799 b. ApJ 723(1):850. https://doi.org/10.1088/0004-637X/723/1/850

Bracewell RN (1978) Detecting nonsolar planets by spinning infrared interferometer. Nature 274:780. https://doi.org/10.1038/274780a0

Breckinridge J, Lindensmith C (2005) The astronomical search for origins. Opt Photon News 16:24. https://doi.org/10.1364/OPN.16.2.000024

Brown G, Thomson RR, Kar AK, Psaila ND, Bookey HT (2012) Ultrafast laser inscription of Bragggrating waveguides using the multiscan technique. Opt Lett 37(4):491-493. https://doi.org/10.1364/ OL.37.000491

Brustlein S, Del Rio L, Tonello A, Delage L, Reynaud F, Herrmann H, Sohler W (2008) Laboratory demonstration of an infrared-to-visible up-conversion interferometer for spatial coherence analysis. Phys Rev Lett 100(15):153903. https://doi.org/10.1103/PhysRevLett.100.153903

Bryant JJ, O'Byrne JW, Bland-Hawthorn J, Leon-Saval SG (2011) Characterization of hexabundles: initial results. MNRAS 415(3):2173-2181. https://doi.org/10.1111/j.1365-2966.2011.18841.X

Bryant JJ, Owers MS, Robotham ASG, Croom SM, Driver SP, Drinkwater MJ, Lorente NPF, Cortese L, Scott N, Colless M et al (2015) The SAMI Galaxy Survey: instrument specification and target selection. MNRAS 447(3):2857-2879. https://doi.org/10.1093/mnras/stu2635

Burtscher L, Hönig S, Jaffe W, Kishimoto M, Lopez-Gonzaga N, Meisenheimer K, Tristam KRW (2016) Infrared interferometry and AGNs: Parsec-scale disks and dusty outflows. In: Malbet F, CreechEakman MJ, Tuthill PG (eds) Optical and Infrared Interferometry and Imaging V. Proceedings of SPIE, vol 9907. SPIE, p 99070R. https://doi.org/10.1117/12.2231077. arXiv:1607.04533

Buscher DF, Haniff CA, Baldwin JE, Warner PJ (1990) Detection of a bright feature on the surface of Betelgeuse. MNRAS 245:7P

Buscher DF, Creech-Eakman M, Farris A, Haniff CA, Young JS (2013) The conceptual design of the magdalena ridge observatory interferometer. J Astron Instr 2:1340001. https://doi.org/10.1142/ S2251171713400011. arXiv:1307.0391 [astro-ph.IM]

Butler RP, Marcy GW, Williams E, McCarthy C, Dosanjh P, Vogt SS (1996) Attaining Doppler precision of $3 \mathrm{~m} \mathrm{~s}^{-1}$. PASP 108:500. https://doi.org/10.1086/133755

Calia DB, Hackenberg WK, Araujo C, Guidolin I, Alvarez JL (2004) Laser-guide-star-related activities at ESO. In: Calia DB, Ellerbroek BL, Ragazzoni R (eds) Advancements in Adaptive Optics. Proceedings of SPIE, vol 5490. SPIE. pp 974-980. https://doi.org/10.1117/12.552947

Ceus D, Tonello A, Grossard L, Delage L, Reynaud F, Herrmann H, Sohler W (2011) Phase closure retrieval in an infrared-to-visible upconversion interferometer for high resolution astronomical imaging. Opt Express 19:8616. https://doi.org/10.1364/OE.19.008616

Ceus D, Reynaud F, Woillez J, Lai O, Delage L, Grossard L, Baudoin R, Gomes JT, Bouyeron L, Herrmann H, Sohler W (2012) Application of frequency conversion of starlight to high-resolution imaging interferometry. On-sky sensitivity test of a single arm of the interferometer. MNRAS 427:L95-L98. https://doi.org/10.1111/j.1745-3933.2012.01352.x

Charles N, Jovanovic N, Gross S, Stewart P, Norris B, O’Byrne J, Lawrence JS, Withford MJ, Tuthill PG (2012) Design of optically path-length-matched, three-dimensional photonic circuits comprising uniquely routed waveguides. Appl Opt 51:6489. https://doi.org/10.1364/AO.51.006489. arXiv:1209. 5144 [astro-ph.IM]

Chesneau O, Banerjee DPK, Millour F, Nardetto N, Sacuto S, Spang A, Wittkowski M, Ashok NM, Das RK, Hummel C, Kraus S, Lagadec E, Morel S, Petr-Gotzens M, Rantakyro F, Schöller M (2008) VLTI monitoring of the dust formation event of the Nova V1280 Scorpii. A\&A 487:223-235. https://doi.org/10.1051/0004-6361:200809485. arXiv:0804.4129

Chilcote JK, Larkin JE, Maire J, Perrin MD, Fitzgerald MP, Doyon R, Thibault S, Bauman B, Macintosh BA, Graham JR, Saddlemyer L (2012) Performance of the integral field spectrograph for the Gemini Planet Imager. In: McLean IS, Ramsay SK, Takami H (eds) Ground-based and airborne instrumentation for astronomy IV. Proceedings of SPIE, vol 8446, p 84468W. https://doi.org/10. $1117 / 12.925790$ 
Ciliegi P, Diolaiti E, Abicca R, Agapito G, Aliverti M, Arcidiacono C, Auricchio N, Balestra A, Baruffolo A, Bellazzini M, Bonaglia M, Bregoli G, Brissaud O, Busoni L, Carlotti A, Cascone E, Correia JJ, Cortecchia F, Cosentino G, D’Orazi V, Dall’Ora M, Caprio VD, Rosa AD, Delboulbé A, Antonio ID, Rico GD, Dolci M, Esposito S, Fantinel D, Feautrier P, Fiorentino G, Foppiani I, Giro E, Gluck L, Grani P, Greggio D, Hénault F, Jocou L, Penna PL, Lafrasse S, Lauria M, Coarer EL, Louarn ML, Lombini M, Magnard Y, Magrin D, Maiorano E, Mannucci F, Marchetti E, Maurel D, Michaud L, Moraux E, Morgante G, Moulin T, Oberti S, Pariani G, Patti M, Plantet C, Podio L, Puglisi A, Rabou P, Ragazzoni R, Redaelli E, Riva M, Rochat S, Roussel F, Roux A, Salasnich B, Saracco P, Schreiber L, Spavone M, Stadler E, Sztefek MH, Terenzi L, Valentini A, Ventura N, Vérinaud C, Zaggia S (2018) MAORY for ELT: preliminary design overview. In: Close LM, Schreiber L, Schmidt D (eds) Adaptive optics systems VI. Proceedings of SPIE, vol 10703. SPIE, pp 336-345. https://doi.org/10.1117/12.2313672

Claudi RU, Turatto M, Gratton RG, Antichi J, Bonavita M, Bruno P, Cascone E, De Caprio V, Desidera S, Giro E, Mesa D, Scuderi S, Dohlen K, Beuzit JL, Puget P (2008) SPHERE IFS: the spectro differential imager of the VLT for exoplanets search. In: McLean IS, Casali MM (eds) Groundbased and airborne instrumentation for astronomy II. Proceedings of SPIE, vol 7014. SPIE, p 70143E. https://doi.org/10.1117/12.788366

Cockell CS, Herbst T, Léger A, Absil O, Beichman C, Benz W, Brack A, Chazelas B, Chelli A, Cottin H, Coudédu Foresto V, Danchi W, Defrère D, den Herder JW, Eiroa C, Fridlund M, Henning T, Johnston K, Kaltenegger L, Labadie L, Lammer H, Launhardt R, Lawson P, Lay OP, Liseau R, Martin SR, Mawet D, Mourard D, Moutou C, Mugnier L, Paresce F, Quirrenbach A, Rabbia Y, Rottgering HJA, Rouan D, Santos N, Selsis F, Serabyn E, Westall F, White G, Ollivier M, Bordé P (2009) Darwin-an experimental astronomy mission to search for extrasolar planets. Exp Astron 23(1):435-461. https://doi.org/10.1007/s10686-008-9121-x

Colavita MM, Wallace JK, Hines BE, Gursel Y, Malbet F, Palmer DL, Pan XP, Shao M, Yu JW, Boden AF, Dumont PJ, Gubler J, Koresko CD, Kulkarni SR, Lane BF, Mobley DW, van Belle GT (1999) The Palomar testbed interferometer. ApJ 510(1):505-521. https://doi.org/10.1086/306579

Comte G, Surace C (1994) Slitless spectroscopy by photographic and CCD detectors across large fields. Symposium-International Astronomical Union, vol 161. Cambridge University Press, Cambridge, pp 709-714

Connes P, Froehly C, Facq P (1985) A fiber-linked version of project TRIO. In: Longdon N, Melita O (eds) Kilometric Optical Arrays in Space. ESA Special Publication, vol 226

Connes P, Shaklan S, Roddier F (1987) A Fiber-linked groundbased array. In: Goad JW (ed) Interferometric imaging in astronomy, NOAO

Content R, Blake S, Dunlop C, Nandi D, Sharples R, Talbot G, Shanks T, Donoghue D, Galiatsatos N, Luke P (2013) New microslice technology for hyperspectral imaging. Remote Sens 5(3):1204-1219. https://doi.org/10.3390/rs5031204

Correia S, Zinnecker H, Ratzka T, Sterzik MF (2006) A VLT/NACO survey for triple and quadruple systems among visual pre-main sequence binaries. A\&A 459:909-926. https://doi.org/10.1051/ 0004-6361:20065545. arXiv:astro-ph/0608674

Corrigan M, Harris RJ, Thomson RR, MacLachlan DG, Allington-Smith J, Myers R, Morris T (2016) Wavefront sensing using a photonic lantern. In: Marchetti E, Close LM, Véran JP (eds) Adaptive optics systems V. Proceedings of SPIE, vol 9909. SPIE, p 990969. https://doi.org/10.1117/12. 2230568

Coudé du Foresto V, Ridgway ST (1992) FLUOR: a Stellar Interferometer Using Single-Mode Fibers. In: Beckers JM, Merkle F (eds) ESO Conference on High-Resolution Imaging by Interferometry II: ground-based interferometry at visible and infrared wavelengths, 15-18, (October 1991) European Southern Observatory Conference and Workshop Proceedings, vol 39. Garching, ESO, p 731

Coudé du Foresto V, Perrin G, Mariotti JM, Lacasse M, Traub W (1997a) The FLUOR/IOTA fiber stellar interferometer. In: Kern P, Malbet F (eds) Integrated optics for astronomical interferometry. Bastianelli-Guirimand, Grenoble, pp 115-125

Coudé du Foresto V, Ridgway S, Mariotti JM (1997b) Deriving object visibilities from interferograms obtained with a fiber stellar interferometer. A\&AS. https://doi.org/10.1051/aas:1997290

Coudé du Foresto V, Borde PJ, Merand A, Baudouin C, Remond A, Perrin GS, Ridgway ST, ten Brummelaar TA, McAlister HA (2003) FLUOR fibered beam combiner at the CHARA array. In: Traub WA (ed) Interferometry for optical astronomy II. Proceedings of SPIE, vol 4838. SPIE, pp 280-285. https://doi.org/10.1117/12.459942 
Courtes G (1982) An integral field spectrograph (IFS) for large telescopes. International Astronomical Union Colloquium, vol 67. Cambridge University Press, Cambridge, pp 123-128

Crepp JR, Crass J, King D, Bechter A, Bechter E, Ketterer R, Reynolds R, Hinz P, Kopon D, Cavalieri D, et al (2016) iLocater: a diffraction-limited Doppler spectrometer for the Large Binocular Telescope. In: Evans CJ, Simard L, Takami H (eds) Ground-based and Airborne Instrumentation for Astronomy VI. Proceedings of SPIE, vol 9908. SPIE, p 990819. https://doi.org/10.1117/12.2233135

Croom SM, Lawrence JS, Bland-Hawthorn J, Bryant JJ, Fogarty L, Richards S, Goodwin M, Farrell T, Miziarski S, Heald R et al (2012) The Sydney-AAO Multi-object Integral field spectrograph. MNRAS 421(1):872-893. https://doi.org/10.1111/j.1365-2966.2011.20365.x

Crouzier A, Malbet F, Henault F, Léger A, Cara C, LeDuigou JM, Preis O, Kern P, Delboulbe A, Martin G, Feautrier P, Stadler E, Lafrasse S, Rochat S, Ketchazo C, Donati M, Doumayrou E, Lagage PO, Shao M, Goullioud R, Nemati B, Zhai C, Behar E, Potin S, Saint-Pe M, Dupont J (2016) A detector interferometric calibration experiment for high precision astrometry. A\&A 595:A108. https://doi. org/10.1051/0004-6361/201526321. arXiv:1609.02477 [astro-ph.IM]

Cvetojevic N, Lawrence JS, Ellis SC, Bland-Hawthorn J, Haynes R, Horton A (2009) Characterization and on-sky demonstration of an integrated photonic spectrograph for astronomy. Opt Express 17(21):18643-18650

Cvetojevic N, Jovanovic N, Lawrence J, Withford M, Bland-Hawthorn J (2012) Developing arrayed waveguide grating spectrographs for multi-object astronomical spectroscopy. Opt Express 20(3):2062-2072. https://doi.org/10.1364/OE.20.002062

Cvetojevic N, Jovanovic N, Gross S, Norris B, Spaleniak I, Schwab C, Withford MJ, Ireland M, Tuthill P, Guyon O, Martinache F, Lawrence JS (2017) Modal noise in an integrated photonic lantern fed diffraction-limited spectrograph. Opt Express 25(21):25546-25565. https://doi.org/10.1364/OE.25. 025546

Defrère D, Hinz PM, Mennesson B, Hoffmann WF, Millan-Gabet R, Skemer AJ, Bailey V, Danchi WC, Downey EC, Durney O, Grenz P, Hill JM, McMahon TJ, Montoya M, Spalding E, Vaz A, Absil O, Arbo P, Bailey H, Brusa G, Bryden G, Esposito S, Gaspar A, Haniff CA, Kennedy GM, Leisenring JM, Marion L, Nowak M, Pinna E, Powell K, Puglisi A, Rieke G, Roberge A, Serabyn E, Sosa R, Stapeldfeldt K, Su K, Weinberger AJ, Wyatt MC (2016) Nulling data reduction and on-sky performance of the large binocular telescope interferometer. ApJ 824:66. https://doi.org/10.3847/ 0004-637X/824/2/66. arXiv:1601.06866 [astro-ph.EP]

Dekany R, Roberts J, Burruss R, Bouchez A, Truong T, Baranec C, Guiwits S, Hale D, Angione J, Trinh T, Zolkower J, Shelton JC, Palmer D, Henning J, Croner E, Troy M, McKenna D, Tesch J, Hildebrandt S, Milburn J (2013) PALM-3000: exoplanet adaptive optics for the $5 \mathrm{~m}$ Hale Telescope. ApJ 776(2):130. https://doi.org/10.1088/0004-637X/776/2/130

Delacroix C, Forsberg P, Karlsson M, Mawet D, Absil O, Hanot C, Surdej J, Habraken S (2012) Design, manufacturing, and performance analysis of mid-infrared achromatic half-wave plates with diamond subwavelength gratings. Appl Opt 51:5897. https://doi.org/10.1364/AO.51.005897

Delacroix C, Absil O, Forsberg P, Mawet D, Christiaens V, Karlsson M, Boccaletti A, Baudoz P, Kuittinen M, Vartiainen I, Surdej J, Habraken S (2013) Laboratory demonstration of a mid-infrared AGPM vector vortex coronagraph. A\&A 553:A98

Delplancke F, Derie F, Lévêque S, Ménardi S, Abuter R, Andolfato L, Ballester P, de Jong J, Di Lieto N, Duhoux P, Frahm R, Gitton P, Glindemann A, Palsa R, Puech F, Sahlmann J, Schuhler N, Duc TP, Valat B, Wallander A (2006) PRIMA for the VLTI: a status report. In: Monnier JD, Schöller M, Danchi WC (eds) Advances in stellar interferometry. Proceedings of SPIE, vol 6268, p 62680U. https://doi.org/10.1117/12.660395

Diab M, Minardi S (2018) On the modal throughput of photonic lanterns in the presence of partial adaptive optic correction. In: Navarro R, Geyl R (eds) Advances in optical and mechanical technologies for telescopes and instrumentation III. Proceedings of SPIE, vol 10706, SPIE, p 107064T. https://doi.org/10.1117/12.2309657

Diener R, Tepper J, Labadie L, Pertsch T, Nolte S, Minardi S (2017) Towards 3d-photonic, multitelescope beam combiners for mid-infrared astrointer ferometry. Opt Express 25(16):19262-19274. https://doi.org/10.1364/OE.25.019262

Dietrich PI, Harris RJ, Blaicher M, Corrigan MK, Morris TJ, Freude W, Quirrenbach A, Koos C (2017) Printed freeform lens arrays on multi-core fibers for highly efficient coupling in astrophotonic systems. Opt Express 25(15):18288-18295. https://doi.org/10.1364/OE.25.018288

Do T, Hees A, Ghez A, Martinez GD, Chu DS, Jia S, Sakai S, Lu JR, Gautam AK, O’Neil KK, Becklin EE, Morris MR, Matthews K, Nishiyama S, Campbell R, Chappell S, Chen Z, Ciurlo A, Dehghanfar 
A, Gallego-Cano E, Kerzendorf WE, Lyke JE, Naoz S, Saida H, Schödel R, Takahashi M, Takamori Y, Witzel G, Wizinowich P (2019) Relativistic redshift of the star S0-2 orbiting the galactic center supermassive black hole. Science 365(6454):664-668. https://doi.org/10.1126/science.aav8137

Dos Santos D, Rativa D, Vohnsen B (2015) Wavefront sensing using a liquid-filled photonic crystal fiber. Opt Express 23:13005-13014. https://doi.org/10.1364/OE.23.013005

Douglass G, Dreisow F, Gross S, Withford MJ (2018) Femtosecond laser written arrayed waveguide gratings with integrated photonic lanterns. Opt Express 26(2):1497-1505. https://doi.org/10.1364/ OE.26.001497

Downes RA, Wallace D (1996) Spectroscopy of suspected variable stars. PASP 108(720):134. https://doi. org/10.1086/133702

Drew J, Bergeron J, Bouvier J et al (2010) Report by the European telescope strategic review committee on Europe's 2-4 m telescopes over the decade to 2020. Tech. rep, Astronet

Dubbeldam CM, Allington-Smith JR, Pokrovski S, Robertson DJ (2000) Integral field unit for the Gemini near-infrared spectrograph. In: Iye M, Moorwood AFM (eds) Optical and IR telescope instrumentation and detectors. Proceedings of SPIE, vol 4008. SPIE, pp 1181-1192. https://doi. org/10.1117/12.395437

Duchêne G, McCabe C, Pinte C, Stapelfeldt KR, Ménard F, Duvert G, Ghez AM, Maness HL, Bouy H, Barrado y Navascués D, Morales-Calderón M, Wolf S, Padgett DL, Brooke TY, Noriega-Crespo A, (2010) Panchromatic observations and modeling of the HV Tau C edge-on disk. ApJ 712:112-129. https://doi.org/10.1088/0004-637X/712/1/112. arXiv:0911.3445 [astro-ph.SR]

Duquennoy A, Mayor M (1991) Multiplicity among solar-type stars in the solar neighbourhood. II. Distribution of the orbital elements in an unbiased sample. A\&A 248:485-524

Ellis RS, Parry IR (1988) Multiple object spectroscopy. In: Robinson LB (ed) Instrumentation for groundbased optical astronomy. Present and Future. Springer, New York, pp 192-208. https://doi.org/10. 1007/978-1-4612-3880-5_19

Ellis SC, Crouzier A, Bland-Hawthorn J, Lawrence J (2011) Potential applications of ring resonators for astronomical instrumentation. In: Proceedings of the International Quantum Electronics Conference and Conference on Lasers and Electro-Optics Pacific Rim 2011. OSA, p C677

Ellis SC, Bauer S, Bland-Hawthorn J, Case S, Content R, Fechner T, Giannone D, Haynes R, Hernandez E, Horton AJ, Klauser U, Lawrence JS, Leon-Saval SG, Lindley E, Löhmannsröben HG, Min SS, Pai N, Roth M, Shortridge K, Staszak NF, Tims J, Xavier P, Zhelem R (2016) PRAXIS: a near infrared spectrograph optimised for $\mathrm{OH}$ suppression. In: Evans CJ, Simard L, Takami H (eds) Ground-based and airborne instrumentation for astronomy VI. Proceedings of SPIE, vol 9908. SPIE, p 99084A. https://doi.org/10.1117/12.2232115

Ellis SC, Kuhlmann S, Kuehn K, Spinka H, Underwood D, Gupta RR, Ocola LE, Liu P, Wei G, Stern NP, Bland-Hawthorn J, Tuthill P (2017) Photonic ring resonator filters for astronomical OH suppression. Opt Express 25(14):15868-15889. https://doi.org/10.1364/OE.25.015868

Ellis SC, Bland-Hawthorn J, Lawrence JS, Horton AJ, Content R, Roth MM, Pai N, Zhelem R, Case S, Hernandez E, Leon-Saval SG, Haynes R, Min SS, Giannone D, Madhav K, Rahman A, Betters C, Haynes D, Couch W, Kewley LJ, McDermid R, Spitler L, Sharp RG, Veilleux S (2020) First demonstration of $\mathrm{OH}$ suppression in a high-efficiency near-infrared spectrograph. MNRAS 492(2):2796-2806. https://doi.org/10.1093/mnras/staa028

Errmann R, Minardi S, Pertsch T (2013) A broad-band scalar vortex coronagraph. MNRAS 435:565-569. https://doi.org/10.1093/mnras/stt1317. arXiv:1307.4347 [astro-ph.IM]

Errmann R, Minardi S, Labadie L, Muthusubramanian B, Dreisow F, Nolte S, Pertsch T (2015) Interferometric nulling of four channels with integrated optics. Appl Opt 54:7449. https://doi.org/10. 1364/AO.54.007449

Ertel S, Absil O, Defrère D, Le Bouquin JB, Augereau JC, Marion L, Blind N, Bonsor A, Bryden G, Lebreton J, Milli J (2014) A near-infrared interferometric survey of debris-disk stars. IV. An unbiased sample of 92 southern stars observed in H band with VLTI/PIONIER. Astron Astrophys 570:A128. https://doi.org/10.1051/0004-6361/201424438. arXiv:1409.6143 [astro-ph.EP]

Ertel S, Defrère D, Hinz P, Mennesson B, Kennedy GM, Danchi WC, Gelino C, Hill JM, Hoffmann WF, Mazoyer J, Rieke G, Shannon A, Stapelfeldt K, Spalding E, Stone JM, Vaz A, Weinberger AJ, Willems P, Absil O, Arbo P, Bailey VP, Beichman C, Bryden G, Downey EC, Durney O, Esposito S, Gaspar A, Grenz P, Haniff CA, Leisenring JM, Marion L, McMahon TJ, Millan-Gabet R, Montoya M, Morzinski KM, Perera S, Pinna E, Pott JU, Power J, Puglisi A, Roberge A, Serabyn E, Skemer AJ, Su KYL, Vaitheeswaran V, Wyatt MC (2020) The HOSTS Survey for Exozodiacal 
Dust: Observational Results from the Complete Survey. AJ 159(4):177. https://doi.org/10.3847/ 1538-3881/ab7817. arXiv:2003.03499 [astro-ph.SR]

Evans CJ, Puech M, Rodrigues M, Barbuy B, Cuby JG, Dalton G, Fitzsimons E, Hammer F, Jagourel P, Kaper L, Morris SL, Morris TJ (2016) Science requirements and trade-offs for the MOSAIC instrument for the European ELT. In: Ground-based and airborne instrumentation for astronomy VI. Proceedings of SPIE, vol 9908, p 99089J. https://doi.org/10.1117/12.2231675. arXiv:1608.06542 [astro-ph.IM]

Feger T, Bacigalupo C, Bedding TR, Bento J, Coutts DW, Ireland MJ, Parker QA, Rizzuto A, Spaleniak I (2014) RHEA: the ultra-compact replicable high-resolution exoplanet and asteroseismology spectrograph. In: Ramsay SK, McLean IS, Takami H (eds) Ground-based and airborne instrumentation for astronomy V. Proceedings of SPIE, vol 9147. SPIE, p 91477I. https://doi.org/ 10.1117/12.2055600

Feng Y, Taylor L, Calia DB (2008) Multiwatts narrow line width fiber Raman amplifiers. Opt Express 16:10927-10932. https://doi.org/10.1364/OE.16.010927

Feng Y, Taylor L, Calia DB (2009) 25 W Raman-fiber-amplifier-based $589 \mathrm{~nm}$ laser for laser guide star. Opt Express 17:19021-19026. https://doi.org/10.1364/OE.17.019021

Fizeau H (1868) Prix Bordin: Rapport sur le concours de l'annee 1867. C R Acad Sci 66:932-934

Foo G, Palacios DM, Swartzlander GA (2005) Optical vortex coronagraph. Opt Lett 30:3308-3310. https://doi.org/10.1364/OL.30.003308

Foy R, Labeyrie A (1985) Feasibility of adaptive telescope with laser probe. A\&A 152:L29-L31

Freedman WL, Madore BF, Gibson BK, Ferrarese L, Kelson DD, Sakai S, Mould JR, Kennicutt RC Jr, Ford HC, Graham JA, Huchra JP, Hughes SMG, Illingworth GD, Macri LM, Stetson PB (2001) Final results from the Hubble Space Telescope key project to measure the Hubble constant. ApJ 553:47-72. https://doi.org/10.1086/320638. arXiv:stro-ph/0012376

Fridlund CVM (2004) The Darwin mission. Adv Space Res 34:613-617. https://doi.org/10.1016/j.asr. 2003.05.031

Fried DL (1966) Limiting resolution looking down through the atmosphere. J Opt Soc Am 56:1380. https://doi.org/10.1364/JOSA.56.001380

Froehly C (1981) Coherence and interferometry through optical fibers. In: Ulrich MH, Kjär K (eds) Scientific Importance of High Angular Resolution at Infrared and Wavelenghts. ESO, Garching, pp 285-293

Garufi A, Benisty M, Stolker T, Avenhaus H, de Boer J, Pohl A, Quanz SP, Dominik C, Ginski C, Thalmann C, van Boekel R, Boccaletti A, Henning T, Janson M, Salter G, Schmid HM, Sissa E, Langlois M, Beuzit JL, Chauvin G, Mouillet D, Augereau JC, Bazzon A, Biller B, Bonnefoy M, Buenzli E, Cheetham A, Daemgen S, Desidera S, Engler N, Feldt M, Girard J, Gratton R, Hagelberg J, Keller C, Keppler M, Kenworthy M, Kral Q, Lopez B, Maire AL, Menard F, Mesa D, Messina S, Meyer MR, Milli J, Min M, Muller A, Olofsson J, Pawellek N, Pinte C, Szulagyi J, Vigan A, Wahhaj Z, Waters R, Zurlo A (2017) Three years of SPHERE: the latest view of the morphology and evolution of protoplanetary discs. The Messenger 169:32-37. https://doi.org/10.18727/07226691/5036. arXiv:1710.02795 [astro-ph.EP]

Gatkine P, Veilleux S, Hu Y, Zhu T, Meng Y, Bland-Hawthorn J, Dagenais M (2016) Development of high-resolution arrayed waveguide grating spectrometers for astronomical applications: first results. In: Navarro R, Burge JH (eds) Advances in optical and mechanical technologies for telescopes and instrumentation II. Proceedings of SPIE, vol 9912. SPIE, p 991271. https://doi.org/10.1364/OE.25. 017918

Gatkine P, Veilleux S, Hu Y, Bland-Hawthorn J, Dagenais M (2017) Arrayed waveguide grating spectrometers for astronomical applications: new results. Opt Express 25(15):17918-17935. https:// doi.org/10.1364/OE.25.017918

Gattass RR, Cerami LR, Mazur E (2006) Micromachining of bulk glass with bursts of femtosecond laser pulses at variable repetition rates. Opt Express 14(12):5279-5284

Ge J, Angel JRP, Shelton JC (1998) Optical spectroscopy with a near-single-mode fiber-feed and adaptive optics. In: D’Odorico S (ed) Optical Astronomical Instrumentation. Proceedings of SPIE, vol 3355. SPIE, p 253. https://doi.org/10.1364/OE.14.005279

Ghez AM, Neugebauer G, Matthews K (1993) The multiplicity of T Tauri stars in the star forming regions Taurus-Auriga and Ophiuchus-Scorpius: A 2.2 micron speckle imaging survey. AJ 106:2005-2023. https://doi.org/10.1086/116782 
Gillessen S, Plewa PM, Eisenhauer F, Sari R, Waisberg I, Habibi M, Pfuhl O, George E, Dexter J, von Fellenberg S, Ott T, Genzel R (2017) An update on monitoring stellar orbits in the galactic center. ApJ 837(1):30. https://doi.org/10.3847/1538-4357/aa5c41

Gizis JE, Reid IN, Monet DG (1999) A 2MASS survey for brown dwarfs toward the Hyades. AJ 118(2):997. https://doi.org/10.1086/300982

Glenday AG, Li CH, Langellier N, Chang G, Chen LJ, Furesz G, Zibrov AA, Kärtner F, Phillips DF, Sasselov D, Szentgyorgyi A, Walsworth RL (2015) Operation of a broadband visible-wavelength astro-comb with a high-resolution astrophysical spectrograph. Optica 2(3):250-254. https://doi.org/ 10.1364/OPTICA.2.000250

Goodman JW (2003) Introduction to Fourier optics. Roberts, Englewood, CO

Goodman JW, Rawson EG (1981) Statistics of modal noise in fibers: a case of constrained speckle. Opt Lett 6(7):324-326. https://doi.org/10.1364/OL.6.000324

Goto M, Usuda T, Dullemond CP, Henning T, Linz H, Stecklum B, Suto H (2006) Inner rim of a molecular disk spatially resolved in infrared CO emission lines. ApJ 652:758-762. https://doi.org/ 10.1086/506582. arXiv:astro-ph/0606362

GRAVITY Collaboration, Abuter R, Accardo M, Amorim A, Anugu N, Ávila G, Azouaoui N et al (2017) First light for GRAVITY: Phase referencing optical interferometry for the very large telescope interferometer. A\&A 602:A94. https://doi.org/10.1051/0004-6361/201730838. arXiv:1705.02345 [astro-ph.IM]

GRAVITY Collaboration, Abuter R, Amorim A, Bauböck M, Berger JP, Bonnet H, Brandner W et al (2020) Detection of the Schwarzschild precession in the orbit of the star S2 near the Galactic centre massive black hole. A\&A 636:L5. https://doi.org/10.1051/0004-6361/202037813

Gris-Sánchez I, Haynes DM, Ehrlich K, Haynes R, Birks TA (2018) Multicore fibre photonic lanterns for precision radial velocity Science. MNRAS 475(3):3065-3075. https://doi.org/10.1093/mnras/ stx3278. arXiv:1707.01719 [astro-ph.IM]

Gross S, Jovanovic N, Sharp A, Ireland M, Lawrence J, Withford MJ (2015) Low loss mid-infrared ZBLAN waveguides for future astronomical applications. Opt Express 23:7946. https://doi.org/10. 1364/OE.23.007946

Gurevich YV, Stürmer J, Schwab C, Führer T, Lamoreaux SK, Quirrenbach A, Walther T (2014) A laser locked Fabry-Perot etalon with $3 \mathrm{~cm} / \mathrm{s}$ stability for spectrograph calibration. In: Ramsay SK, McLean IS, Takami H (eds) Ground-based and airborne instrumentation for astronomy V. Proceedings of SPIE, vol 9147. SPIE, p 91477M. https://doi.org/10.1117/12.2057008

Gustafsson M, Labadie L, Herbst TM, Kasper M (2008) Spatially resolved $\mathrm{H}_{2}$ emission from the disk around T Tau N. A\&A 488:235-244. https://doi.org/10.1051/0004-6361:200809770. arXiv:0806. 3084

Guyon O (2003) Phase-induced amplitude apodization of telescope pupils for extrasolar terrestrial planet imaging. A\&A 404:379-387. https://doi.org/10.1051/0004-6361:20030457. arXiv:astro-ph/0301190

Guyon O, Roddier C, Graves JE, Roddier F, Cuevas S, Espejo C, Gonzales S (1999) The nulling stellar coronagaph: laboratory test and performance evaluation. PASP 111:1321-1330

Guyon O, Pluzhnik EA, Kuchner MJ, Collins B, Ridgway ST (2006) Theoretical Limits on Extrasolar Terrestrial Planet Detection with Coronagraphs. ApJS 167:81-99. https://doi.org/10.1086/507630. arXiv:astro-ph/0608506

Guyon O, Matsuo T, Angel R (2009) Coronagraphic low-order wave-front sensor: principle and application to a phase-induced amplitude coronagraph. ApJ 693:75-84. https://doi.org/10.1088/ 0004-637X/693/1/75. arXiv:0911.1301 [astro-ph.IM]

Haffert SY, Harris RJ, Zanutta A, Pike FA, Bianco A, Redaelli E, Benoît A, MacLachlan DG, Ross CA, Gris-Sánchez I et al (2020) Diffraction-limited integral-field spectroscopy for extreme adaptive optics systems with the multicore fiber-fed integral-field unit. J Astron Telescop Instr Syst 6(4):045007

Haguenauer P, Serabyn E (2006) Deep nulling of laser light with a single-mode-fiber beam combiner. Appl Opt 45:2749-2754. https://doi.org/10.1364/AO.45.002749

Halverson S, Mahadevan S, Ramsey LW, Redman S, Nave G, Wilson JC, Hearty F, Holtzman J (2013) Development of a new, precise near-infrared Doppler wavelength reference: a fiber Fabry-Perot interferometer. In: McLean IS, Ramsay SK, Takami H (eds) Ground-based and Airborne Instrumentation for Astronomy IV. Proceedings of SPIE, vol 8446. SPIE, pp 1198-1213. https:// doi.org/10.1117/12.925716 
Halverson S, Roy A, Mahadevan S, Schwab C (2015) 'modal noise' in single-mode fibers: a cautionary note for high precision radial velocity instruments. ApJ 814(2):L22. https://doi.org/10.1088/2041$8205 / 814 / 2 / 122$

Haniff CA, Mackay CD, Titterington DJ, Sivia D, Baldwin JE (1987) The first images from optical aperture synthesis. Nature 328:694-696. https://doi.org/10.1038/328694a0

Hankla AK, Bartholomew J, Groff K, Lee I, McKinnie IT, Moule G, Rogers N, Tiemann B, Tracy AJ, VanHoudt P, Adkins SM, d'Orgeville C (2006) $20 \mathrm{~W}$ and $50 \mathrm{~W}$ solid-state sodium beacon guidestar laser systems for the Keck I and Gemini South telescopes. In: Ellerbroek BL, Calia DB (eds) Advances in adaptive optics II. Proceedings of SPIE, vol 6272. SPIE, p 62721G. https://doi.org/10. $1117 / 12.670054$

Hanot C, Mennesson B, Martin S, Liewer K, Loya F, Mawet D, Riaud P, Absil O, Serabyn E (2011) Improving interferometric null depth measurements using statistical distributions: theory and first results with the Palomar Fiber Nuller. ApJ 729:110. https://doi.org/10.1088/0004-637X/729/2/110. arXiv:1103.4719 [astro-ph.IM]

Harris RJ, Allington-Smith JR (2012) Applications of integrated photonic spectrographs in astronomy. MNRAS 428(4):3139-3150. https://doi.org/10.1093/mnras/sts265

Harris RJ, MacLachlan DG, Choudhury D, Morris TJ, Gendron E, Basden AG, Brown G, AllingtonSmith JR, Thomson RR (2015) Photonic spatial reformatting of stellar light for diffraction-limited spectroscopy. MNRAS 450(1):428-434. https://doi.org/10.1093/mnras/stv410

Hartman JD, Bakos G, Noyes RW, Sipőcz B, Kovács G, Mazeh T, Shporer A, Pál A (2011) A photometric variability survey of field $\mathrm{k}$ and $\mathrm{m}$ dwarf stars with HATnet. AJ 141(5):166. https://doi. org/10.1088/0004-6256/141/5/166

Haynes DM, Gris-Sanchez I, Ehrlich K, Birks TA, Giannone D, Haynes R (2014) New multicore low mode noise scrambling fiber for applications in high-resolution spectroscopy. In: Navarro R, Cunningham CR, Barto AA (eds) Advances in optical and mechanical technologies for telescopes and instrumentation. Proceedings of SPIE, vol 9151. SPIE, p 915155. https://doi.org/10.1117/12. 2057170

Haynes DM, Gris-Sanchez I, Birks TA, Haynes R (2018) Optical fiber modal noise suppression in the NIR region using multicore fiber and photonic lanterns. In: Proceedings of SPIE. vol 10706, p 1070665. https://doi.org/10.1117/12.2314224

Heacox WD (1986) On the application of optical-fiber image scramblers to astronomical spectroscopy. AJ 92:219-229

Heacox WD (1988) Wavelength-precise slit spectroscopy with optical fiber image scramblers. In: Barden SC (ed) Fiber Optics in Astronomy, ASP Conference Proceedings, vol 3. Astronomical Society of the Pacific, San Francisco. pp 204-235

Hecht E (2012) Optics. Pearson, Boston

Henning T, Semenov D (2013) Chemistry in protoplanetary disks. Chem Rev 113:9016-9042. https://doi. org/10.1021/cr400128p. arXiv:1310.3151 [astro-ph.GA]

Herr T, Hartinger K, Riemensberger J, Wang CY, Gavartin E, Holzwarth R, Gorodetsky ML, Kippenberg TJ (2012) Universal formation dynamics and noise of Kerr-frequency combs in microresonators. Nature Photon 6(7):480-487. https://doi.org/10.1038/nphoton.2012.127

Herr T, Brasch V, Jost JD, Wang CY, Kondratiev NM, Gorodetsky ML, Kippenberg TJ (2014) Temporal solitons in optical microresonators. Nat Photon 8(2):145-152. https://doi.org/10.1038/nphoton.2013. 343

Hill GJ, MacQueen PJ, Tejada C, Cobos FJ, Palunas P, Gebhardt K, Drory N (2004) VIRUS: a massively replicated IFU spectrograph for HET. In: Moorwood AFM, Iye M (eds) Ground-based Instrumentation for Astronomy. Proceedings of SPIE, vol 5492. SPIE, p 5492. https://doi.org/10. $1117 / 12.552474$

Hill JM, Angel JRP, Scott JS, Lindley D, Hintzen P (1980) Multiple object spectroscopy: the Medusa spectrograph. ApJL 242:L69-L72. https://doi.org/10.1086/183405

Hoeijmakers HJ, Schwarz H, Snellen IAG, de Kok RJ, Bonnefoy M, Chauvin G, Lagrange AM, Girard JH (2018) Medium-resolution integral-field spectroscopy for high-contrast exoplanet imaging. Molecule maps of the $\beta$ Pictoris system with SINFONI. A\&A 617:A144. https://doi.org/10.1051/ 0004-6361/201832902. arXiv:1802.09721 [astro-ph.EP]

Hönig SF, Watson D, Kishimoto M, Hjorth J (2014) A dust-parallax distance of 19 megaparsecs to the supermassive black hole in NGC 4151. Nature 515:528-530. https://doi.org/10.1038/nature13914. arXiv: 1411.7032 
Horton A, Ellis S, Lawrence J, Bland-Hawthorn J (2012) PRAXIS: a low background NIR spectrograph for fibre Bragg grating $\mathrm{OH}$ suppression. In: Navarro R, Cunningham CR, Prieto E (eds) Modern technologies in space- and ground-based telescopes and instrumentation II. Proceedings of SPIE, vol 8450. SPIE. https://doi.org/10.1117/12.924874

Hottinger P, Harris RJ, Dietrich PI, Blaicher M, Glück M, Bechter A, Crass J, Pott JU, Koos C, Sawodny O, Quirrenbach A (2018) Micro-lens arrays as tip-tilt sensor for single mode fiber coupling. In: Navarro R, Geyl R (eds) Advances in optical and mechanical technologies for telescopes and instrumentation III. Proceedings of SPIE, vol 10706. SPIE, p 1070629. https://doi.org/10.1117/12. 2312015. arXiv:1808.03190 [astro-ph.IM]

Hsiao HK, Winick KA, Monnier JD, Berger JP (2009) An infrared integrated optic astronomical beam combiner for stellar interferometry at 3-4 $\mu \mathrm{m}$. Opt Express 17:18489-18500 arXiv:0911.1106 [astro-ph.IM]

Hubbard EN, Angel JR, Gresham MS (1979) Operation of a long fused silica fiber as a link between telescope and spectrograph. ApJ 229:1074-1078

Huby E, Perrin G, Marchis F, Lacour S, Kotani T, Duchêne G, Choquet E, Gates EL, Woillez JM, Lai O, Fédou P, Collin C, Chapron F, Arslanyan V, Burns KJ (2012) FIRST, a fibered aperture masking instrument. I. First on-sky test results. A\&A 541:A55. https://doi.org/10.1051/0004-6361/ 201118517. arXiv:1203.5075 [astro-ph.IM]

Huby E, Duchêne G, Marchis F, Lacour S, Perrin G, Kotani T, Choquet É, Gates EL, Lai O, Allard F (2013) FIRST, a fibered aperture masking instrument. II. Spectroscopy of the Capella binary system at the diffraction limit. A\&A 560:A113. https://doi.org/10.1051/0004-6361/201321894. arXiv:1310. 7879 [astro-ph.IM]

Huby E, Bottom M, Femenia B, Ngo H, Mawet D, Serabyn E, Absil O (2017) On-sky performance of the QACITS pointing control technique with the Keck/NIRC2 vortex coronagraph. A\&A 600:A46. https://doi.org/10.1051/0004-6361/201630232. arXiv:1701.06397 [astro-ph.IM]

Iuzzolino M, Tozzi A, Sanna N, Zangrilli L, Oliva E (2014) Preliminary results on the characterization and performances of ZBLAN fiber for infrared spectrographs. In: Ramsay SK, McLean IS, Takami $\mathrm{H}$ (eds) Ground-based and airborne instrumentation for astronomy V. vol 9147. International SPIE, p 914766

Iwamuro F, Maihara T, Oya S, Tsukamoto H, Hall DNB, Cowie LL, Tokunaga AT, Pickles AJ (1994) Development of an $\mathrm{OH}$-airglow suppressor spectrograph. PASJ 46:515-521

Janson M, Bergfors C, Goto M, Brandner W, Lafrenière D (2010) Spatially resolved spectroscopy of the exoplanet HR 8799 c. ApJL 710:L35-L38. https://doi.org/10.1088/2041-8205/710/1/L35. arXiv: 1001.2017 [astro-ph.EP]

Jenkins C (2008) Optical vortex coronagraphs on ground-based telescopes. MNRAS 384:515-524. https://doi.org/10.1111/j.1365-2966.2007.12744.x. arXiv:0709.0153

Jeys TH, Brailove AA, Mooradian A (1989) Sum frequency generation of sodium resonance radiation. Appl Opt 28:2588-2591. https://doi.org/10.1364/AO.28.002588

Jovanovic N, Tuthill PG, Norris B, Gross S, Stewart P, Charles N, Lacour S, Ams M, Lawrence JS, Lehmann A, Niel C, Robertson JG, Marshall GD, Ireland M, Fuerbach A, Withford MJ (2012) Starlight demonstration of the Dragonfly instrument: an integrated photonic pupil-remapping interferometer for high-contrast imaging. MNRAS 427:806-815. https://doi.org/10.1111/j.13652966.2012.21997.x. arXiv:1210.0603 [astro-ph.IM]

Jovanovic N, Guyon O, Martinache F, Clergeon C, Singh G, Kudo T, Newman K, Kuhn J, Serabyn E, Norris B, Tuthill P, Stewart P, Huby E, Perrin G, Lacour S, Vievard S, Murakami N, Fumika O, Minowa Y, Hayano Y, White J, Lai O, Marchis F, Duchene G, Kotani T, Woillez J (2014) Development and recent results from the Subaru coronagraphic extreme adaptive optics system. In: McLean IS, Ramsay SK, Takami H (eds) Ground-based and Airborne Instrumentation for Astronomy V. Proceedings of SPIE, vol 9147. SPIE, p 91471Q. https://doi.org/10.1117/12.2057249

Jovanovic N, Martinache F, Guyon O, Clergeon C, Singh G, Kudo T, Garrel V, Newman K, Doughty D, Lozi J, Males J, Minowa Y, Hayano Y, Takato N, Morino J, Kuhn J, Serabyn E, Norris B, Tuthill P, Schworer G, Stewart P, Close L, Huby E, Perrin G, Lacour S, Gauchet L, Vievard S, Murakami N, Oshiyama F, Baba N, Matsuo T, Nishikawa J, Tamura M, Lai O, Marchis F, Duchene G, Kotani T, Woillez J (2015) The Subaru coronagraphic extreme adaptive optics system: enabling high-contrast imaging on solar-system scales. PASP 127:890. https://doi.org/10.1086/682989. arXiv:1507.00017 [astro-ph.IM]

Jovanovic N, Cvetojevic N, Norris B, Betters C, Schwab C, Lozi J, Guyon O, Gross S, Martinache F, Tuthill P, Doughty D, Minowa Y, Takato N, Lawrence J (2017a) Demonstration of an efficient, 
photonic-based astronomical spectrograph on an 8-m telescope. Opt Express 25(15):17753-17766. https://doi.org/10.1364/OE.25.017753

Jovanovic N, Schwab C, Guyon O, Lozi J, Cvetojevic N, Martinache F, Leon-Saval S, Norris B, Gross S, Doughty D et al (2017b) Efficient injection from large telescopes into single-mode fibres: enabling the era of ultra-precision astronomy. A\&A 604:A122. https://doi.org/10.1051/0004-6361/ 201630351

Jurgenson C, Fischer D, McCracken T, Sawyer D, Szymkowiak A, Davis A, Muller G, Santoro F (2016) EXPRES: a next generation RV spectrograph in the search for earth-like worlds. In: Evans CJ, Simard L, Takami H (eds) Ground-based and Airborne Instrumentation for Astronomy VI. Proceedings of SPIE, vol 9908. SPIE, p 99086T. https://doi.org/10.1117/12.2233002

Kalas P, Graham JR, Clampin M (2005) A planetary system as the origin of structure in Fomalhaut's dust belt. Nature 435:1067-1070. https://doi.org/10.1038/nature03601. arXiv:astro-ph/0506574

Kalas P, Duchene G, Fitzgerald MP, Graham JR (2007) Discovery of an extended debris disk around the F2 V star HD 15745. ApJL 671:L161-L164. https://doi.org/10.1086/525252. arXiv:0712.0378

Kalas P, Graham JR, Chiang E, Fitzgerald MP, Clampin M, Kite ES, Stapelfeldt K, Marois C, Krist J (2008) Optical images of an exosolar planet 25 light-years from earth. Science 322:1345. https://doi. org/10.1126/science.1166609. arXiv:0811.1994

Kammerer J, Quanz SP (2018) Simulating the exoplanet yield of a space-based mid-infrared interferometer based on Kepler statistics. A\&A 609:A4. https://doi.org/10.1051/0004-6361/ 201731254. arXiv:1707.06820 [astro-ph.EP]

Kenchington Goldsmith HD, Cvetojevic N, Ireland M, Ma P, Tuthill P, Eggleton B, Lawrence JS, Debbarma S, Luther-Davies B, Madden SJ (2016) Chalcogenide glass planar MIR couplers for future chip based Bracewell interferometers. In: Malbet F, Creech-Eakman MJ, Tuthill PG (eds) Optical and Infrared Interferometry and Imaging V. Proceedings of SPIE, vol 9907. SPIE, p 990730. https://doi.org/10.1117/12.2232199. arXiv:1608.04438 [astro-ph.IM]

Kenchington Goldsmith HD, Cvetojevic N, Ireland M, Madden S (2017a) Fabrication tolerant chalcogenide mid-infrared multimode interference coupler design with applications for Bracewell nulling interferometry. Opt Express 25:3038. https://doi.org/10.1364/OE.25.003038. arXiv:1702. 00468 [astro-ph.IM]

Kenchington Goldsmith HD, Ireland M, Ma P, Cevetojevic N, Madden S (2017b) Improving the extinction bandwidth of MMI chalcogenide photonic chip based MIR nulling interferometers. Opt Express 25:16813. https://doi.org/10.1364/OE.25.016813

Kern P, Malbet F, Schanen-Duport I, Benech P (1997) Integrated optics single-mode interferometric beam combiner for near infrared astronomy. In: Integrated optics for astronomical interferometry, Grenoble, France: Bastianelli-Guirimand, Grenoble, p 115

Kern P, Le Coärer E, Benech P (2009) On-chip spectro-detection for fully integrated coherent beam combiners. Opt Express 17:1976-1987. https://doi.org/10.1364/OE.17.001976. arXiv:0902.1688 [astro-ph.IM]

Kervella P, Coudé du Foresto V, Glindemann A, Hofmann R (2000) VINCI: the VLT Interferometer commissioning instrument. In: Léna P, Quirrenbach A (eds) Interferometry in Optical Astronomy. Proceedings of SPIE, vol 4006. SPIE, pp 31-42. https://doi.org/10.1117/12.390227

Kimura M, Maihara T, Iwamuro F, Akiyama M, Tamura N, Dalton GB, Takato N, Tait P, Ohta K, Eto S et al (2010) Fibre multi-object spectrograph (FMOS) for the Subaru Telescope. PASJ 62(5):1135-1147. https://doi.org/10.1093/pasj/62.5.1135

Kloppenborg B, Stencel R, Monnier JD, Schaefer G, Zhao M, Baron F, McAlister H, ten Brummelaar TA, Che X, Farrington C, Pedretti E, Sallave-Goldfinger PJ, Sturmann J, Sturmann L, Thureau N, Turner $\mathrm{N}$, Carroll SM (2010) Infrared images of the transiting disk in the $\epsilon$ Aurigae system. Nature 464:870-872. https://doi.org/10.1038/nature08968

Kotani T, Perrin G, Vergnole S, Woillez J, Guerin J (2005) Characterization of fluoride fibers for the optical hawaiian array for nanoradian astronomy project. Appl Opt 44:5029-5035. https://doi.org/ 10.1364/AO.44.005029

Kraus AL, Ireland MJ (2012) LkCa 15: a young exoplanet caught at formation? ApJ 745:5. https://doi. org/10.1088/0004-637X/745/1/5. arXiv:1110.3808 [astro-ph.EP]

Kraus S, Monnier JD, Ireland MJ, Duchêne G, Espaillat C, Hönig S, Juhasz A, Mordasini C, Olofsson J, Paladini C, Stassun K, Turner N, Vasisht G, Harries TJ, Bate MR, Gonzalez JF, Matter A, Zhu Z, Panic O, Regaly Z, Morbidelli A, Meru F, Wolf S, Ilee J, Berger JP, Zhao M, Kral Q, Morlok A, Bonsor A, Ciardi D, Kane SR, Kratter K, Laughlin G, Pepper J, Raymond S, Labadie L, Nelson RP, Weigelt G, ten Brummelaar T, Pierens A, Oudmaijer R, Kley W, Pope B, Jensen ELN, Bayo A, 
Smith M, Boyajian T, Quiroga-Nuñez LH, Millan-Gabet R, Chiavassa A, Gallenne A, Reynolds M, de Wit WJ, Wittkowski M, Millour F, Gandhi P, Ramos Almeida C, Alonso Herrero A, Packham C, Kishimoto M, Tristram KRW, Pott JU, Surdej J, Buscher D, Haniff C, Lacour S, Petrov R, Ridgway S, Tuthill P, van Belle G, Armitage P, Baruteau C, Benisty M, Bitsch B, Paardekooper SJ, Pinte C, Masset F, Rosotti G (2016) Planet Formation Imager (PFI): science vision and key requirements. In: Malbet F, Creech-Eakman MJ, Tuthill PG (eds) Optical and Infrared Interferometry and Imaging V. Proceedings of SPIE, vol 9907. SPIE, p 99071K. https://doi.org/10.1117/12.2231067. arXiv:1608. 00578 [astro-ph.IM]

Kuhn TS (1962) The structure of scientific revolutions. The University of Chicago Press, Chicago

Labadie L, Wallner O (2009) Mid-infrared guided optics: a perspective for astronomical instruments. Opt Express 17:1947-1962. https://doi.org/10.1364/OE.17.001947. arXiv:0902.0785 [astro-ph.IM]

Labadie L, Le Coarer E, Maurand R, Labeye P, Kern P, Arezki B, Broquin JE (2007) Mid-infrared laser light nulling experiment using single-mode conductive waveguides. A\&A 471:355-360. https://doi. org/10.1051/0004-6361:20067005. arXiv:0705.0146

Labadie L, Kern P, Labeye P, Lecoarer E, Vigreux-Bercovici C, Pradel A, Broquin JE, Kirschner V (2008) Technology challenges for space interferometry: the option of mid-infrared integrated optics. Adv Space Res 41:1975-1982. https://doi.org/10.1016/j.asr.2007.07.013. arXiv:0707.1701

Labadie L, Martin G, Anheier NC, Arezki B, Qiao HA, Bernacki B, Kern P (2011) First fringes with an integrated-optics beam combiner at $10 \mu \mathrm{m}$. A new step towards instrument miniaturization for midinfrared interferometry. A\&A 531:A48-A54. https://doi.org/10.1051/0004-6361/201116727

Labadie L, Minardi S, Tepper J, Diener R, Muthusubramanian B, Pott JU, Gross S, Arriola A, Withford M (2018) Photonics-based mid-infrared interferometry: 4-year results of the ALSI project and future prospects. In: Creech-Eakman MJ, Tuthill PG, Mérand A (eds) Optical and infrared interferometry and imaging VI. Proceedings of SPIE, vol 10701. SPIE, p 107011R. https://doi.org/10.1117/12. 2312930

Lacour S, Thiébaut E, Perrin G (2007) High dynamic range imaging with a single-mode pupil remapping system: a self-calibration algorithm for redundant interferometric arrays. MNRAS 374:832-846. https://doi.org/10.1111/j.1365-2966.2006.11198.x. arXiv:astro-ph/0610458

Lacour S, Lapeyrère V, Gauchet L, Arroud S, Gourgues R, Martin G, Heidmann S, Haubois X, Perrin G (2014) CubeSats as pathfinders for planetary detection: the FIRST-S satellite. In: Oschmann Jr JM, Clampin M, Fazio GG, MacEwen HA (eds) Space telescopes and instrumentation 2014: optical, infrared, and millimeter wave. Proceedings of SPIE, vol 9143. SPIE, p 91432N. https://doi.org/10. 1117/12.2057381. arXiv:1407.4294 [astro-ph.IM]

Lagrange AM, Gratadour D, Chauvin G, Fusco T, Ehrenreich D, Mouillet D, Rousset G, Rouan D, Allard F, Gendron É, Charton J, Mugnier L, Rabou P, Montri J, Lacombe F (2009) A probable giant planet imaged in the $\beta$ Pictoris disk. VLT/NaCo deep L'-band imaging. A\&A 493:L21-L25. https://doi. org/10.1051/0004-6361:200811325. arXiv:0811.3583

Langrock C, Diamanti E, Roussev RV, Yamamoto Y, Fejer MM, Takesue H (2005) Highly efficient single-photon detection at communication wavelengths by use of upconversion in reverse-protonexchanged periodically poled $\mathrm{LiNbO}_{3}$ waveguides. Opt Lett 30:1725-1727. https://doi.org/10.1364/ OL. 30.001725

Larkin JE, Moore AM, Barton EJ, Bauman B, Bui K, Canfield J, Crampton D, Delacroix A, Fletcher M, Hale D, et al. (2010) The infrared imaging spectrograph (IRIS) for TMT: instrument overview. In: McLean IS, Ramsay SK, Takami H (eds) Ground-based and Airborne Instrumentation for Astronomy III. Proceedings of SPIE, p 773529. https://doi.org/10.1117/12.856305

Laurent F, Adjali L, Arns J, Bacon R, Boudon D, Caillier P, Daguisé E, Delabre B, Dubois JP, Godefroy P, Jarno A, Jorden P, Kosmalski J, Lapére V, Lizon JL, Loupias M, Pecontal A, Reiss R, Remillieux A, Renault E, Rupprecht G, Salaun Y (2010) MUSE integral field unit: Test results on the first out of 24. In: Atad-Ettedgui E, Lemke D (eds) Modern technologies in space- and ground-based telescopes and instrumentation. Proceedings of SPIE, vol 7739. SPIE, p 77394M. https://doi.org/10.1117/12. 857004

Lawrence J, Bland-Hawthorn J, Bryant J, Brzeski J, Colless M, Croom S, Gers L, Gilbert J, Gillingham P, Goodwin M, Heijmans J, Horton A, Ireland M, Miziarski S, Saunders W, Smith G (2012) Hector: a high-multiplex survey instrument for spatially resolved galaxy spectroscopy. In: McLean IS, Ramsay SK, Takami H (eds) Ground-based and Airborne Instrumentation for Astronomy IV. Proc. SPIE. , vol 8446. SPIE, p 844653. https://doi.org/10.1117/12.925260

Lazareff B, Berger JP, Kluska J, Le Bouquin JB, Benisty M, Malbet F, Koen C, Pinte C, Thi WF, Absil O, Baron F, Delboulbé A, Duvert G, Isella A, Jocou L, Juhasz A, Kraus S, Lachaume R, Ménard F, 
Millan-Gabet R, Monnier JD, Moulin T, Perraut K, Rochat S, Soulez F, Tallon M, Thiébaut E, Traub W, Zins G (2017) Structure of Herbig AeBe disks at the milliarcsecond scale. A statistical survey in the $\mathrm{H}$ band using PIONIER-VLTI. A\&A 599:A85. https://doi.org/10.1051/0004-6361/ 201629305. arXiv:1611.08428 [astro-ph.SR]

Le Coarer E, Blaize S, Benech P, Stefanon I, Morand A, Lérondel G, Leblond G, Kern P, Fedeli JM, Royer P (2007) Wavelength-scale stationary-wave integrated Fourier-transform spectrometry. Nat Photon 1:473-478. https://doi.org/10.1038/nphoton.2007.138. arXiv:0708.0272 [physics.optics]

Le Fèvre O, Saïsse M, Mancini D, Brau-Nogué S, Caputi O, Castinel L, d'Odorico S, Garilli B, Kissler M, Lucuix C, et al. (2003) Commissioning and performances of the VLT-VIMOS. In: Iye M, Moorwood AFM (eds) Instrument Design and Performance for Optical/Infrared Ground-based Telescopes. Proceedings of SPIE, vol 4841. SPIE, p 1670. https://doi.org/10.1117/12.460959

Lee C, Chu ST, Little BE, Bland-Hawthorn J, Leon-Saval S (2012) Portable frequency combs for optical frequency metrology. Opt Express 20:16671. https://doi.org/10.1364/OE.20.016671

Lee JH, Foo G, Johnson EG, Swartzlander GAJ (2006) Experimental verification of an optical vortex coronagraph. Phys Rev Lett 97:053901

Lehmann L, Darré P, Boulogne H, Delage L, Grossard L, Reynaud F (2018a) Multichannel spectral mode of the ALOHA up-conversion interferometer. MNRAS 477(1):190-194. https://doi.org/10.1093/ mnras/sty648

Lehmann L, Darré P, Szemendera L, Gomes JT, Baudoin R, Ceus D, Brustlein S, Delage L, Grossard L, Reynaud F (2018b) ALOHA - astronomical light optical hybrid analysis. From experimental demonstrations to a MIR instrument proposal. Exp Astron 46(3):447-456. https://doi.org/10.1007/ s10686-018-9585-2

Lehmann L, Delage L, Grossard L, Reynaud F, Golden S, Woods C, Webster L, Sturmann J, ten Brummelaar T (2019) Environmental characterisation and stabilisation of a $2 \times 200$-meter outdoor fibre interferometer at the CHARA Array. Exp Astron 47(3):303-312. https://doi.org/10.1007/ s10686-019-09627-X

Leinert C, Zinnecker H, Weitzel N, Christou J, Ridgway ST, Jameson R, Haas M, Lenzen R (1993) A systematic approach for young binaries in Taurus. A\&A 278:129-149

Lemke U, Corbett J, Allington-Smith J, Murray G (2011) Modal noise prediction in fibre spectroscopy - I. Visibility and the coherent model. MNRAS 417(1):689-697. https://doi.org/10.1111/j.1365-2966. 2011.19312.x

Leon-Saval SG, Birks TA, Bland-Hawthorn J, Englund M (2005) Multimode fiber devices with singlemode performance. Opt Lett 30(19):2545-2547. https://doi.org/10.1364/OL.30.002545

Leon-Saval SG, Betters CH, Bland-Hawthorn J (2012) The Photonic TIGER: a multicore fiber-fed spectrograph. In: Navarro R, Cunningham CR, Prieto E (eds) Modern technologies in space- and ground-based telescopes and instrumentation II. Proceedings of SPIE, vol 8450. SPIE, p 84501K. https://doi.org/10.1117/12.925254

Leon-Saval SG, Betters CH, Salazar-Gil JR, Min SS, Gris-Sanchez I, Birks TA, Lawrence J, Haynes R, Haynes D, Roth M, Veilleux S, Bland-Hawthorn J (2017) Divide and conquer: an efficient solution to highly multimoded photonic lanterns from multicore fibres. Opt Express 25(15):17530-17540. https://doi.org/10.1364/OE.25.017530

Lindley E, Min SS, Leon-Saval S, Cvetojevic N, Lawrence J, Ellis S, Bland-Hawthorn J (2014) Demonstration of uniform multicore fiber Bragg gratings. Opt Express 22(25):31575-31581

Lippa M, Gillessen S, Blind N, Kok Y, Yazıcı Ş, Weber J, Pfuhl O, Haug M, Kellner S, Wieprecht E, Eisenhauer F, Genzel R, Hans O, Haußmann F, Huber D, Kratschmann T, Ott T, Plattner M, Rau C, Sturm E, Waisberg I, Wiezorrek E, Perrin G, Perraut K, Brandner W, Straubmeier C, Amorim A (2016) The metrology system of the VLTI instrument GRAVITY. In: Malbet F, Creech-Eakman MJ, Tuthill PG (eds) Optical and infrared interferometry and imaging V. Proceedings of SPIE, vol 9907. SPIE, p 990722. https://doi.org/10.1117/12.2232272. arXiv:1608.04888 [astro-ph.IM]

Lozi J, Martinache F, Guyon O (2009) Phase-induced amplitude apodization on centrally obscured pupils: design and first laboratory demonstration for the Subaru Telescope pupil. PASP 121:1232. https:// doi.org/10.1086/648392. arXiv:0903.5001 [astro-ph.IM]

Ma P, Choi DY, Yu Y, Gai X, Yang Z, Debbarma S, Madden S, Luther-Davies B (2013) Low-loss chalcogenide waveguides for chemical sensing in the mid-infrared. Opt Express 21:29927. https:// doi.org/10.1364/OE.21.029927

MacLachlan DG, Harris RJ, Choudhury D, Simmonds RD, Salter PS, Booth MJ, Allington-Smith JR, Thomson RR (2016a) Development of integrated mode reformatting components for diffractionlimited spectroscopy. Opt Lett 41(1):76-79. https://doi.org/10.1364/OL.41.000076 
MacLachlan DG, Harris RJ, Gris-Sánchez I, Morris TJ, Choudhury D, Gendron E, Basden AG, Spaleniak I, Arriola A, Birks TA et al (2016b) Efficient photonic reformatting of celestial light for diffractionlimited spectroscopy. MNRAS 464(4):4950-4957. https://doi.org/10.1093/mnras/stw2558

Malbet F, Léger A, Shao M, Goullioud R, Lagage PO, Brown AGA, Cara C, Durand G, Eiroa C, Feautrier P, Jakobsson B, Hinglais E, Kaltenegger L, Labadie L, Lagrange AM, Laskar J, Liseau R, Lunine J, Maldonado J, Mercier M, Mordasini C, Queloz D, Quirrenbach A, Sozzetti A, Traub W, Absil O, Alibert Y, Andrei AH, Arenou F, Beichman C, Chelli A, Cockell CS, Duvert G, Forveille T, Garcia PJV, Hobbs D, Krone-Martins A, Lammer H, Meunier N, Minardi S, Moitinho de Almeida A, Rambaux N, Raymond S, Röttgering HJA, Sahlmann J, Schuller PA, Ségransan D, Selsis F, Surdej J, Villaver E, White GJ, Zinnecker H (2012) High precision astrometry mission for the detection and characterization of nearby habitable planetary systems with the Nearby Earth Astrometric Telescope (NEAT). Exp Astron 34:385-413. https://doi.org/10.1007/s10686-011-9246-1. arXiv:1107.3643 [astro-ph.EP]

Mariotti JM, Coudé du Foresto V, Perrin G, Zhao P, Lena P (1996) Interferometric connection of large ground-based telescopes. A\&AS 116:381-393

Marois C, Macintosh B, Barman T, Zuckerman B, Song I, Patience J, Lafrenière D, Doyon R (2008) Direct imaging of multiple planets orbiting the star HR 8799. Science 322:1348. https://doi.org/10. 1126/science.1166585. arXiv:0811.2606

Martin G, Pugnat T, Gardillou F, Cassagnettes C, Barbier D, Guyot C, Hauden J, Huby E, Lacour S (2016) Novel multi-telescopes beam combiners for next generation instruments (FIRST/SUBARU). In: Malbet F, Creech-Eakman MJ, Tuthill PG (eds) Optical and infrared interferometry and imaging V. Proceedings of SPIE, vol 9907. SPIE, pp 764-771. https://doi.org/10.1117/12.2233105

Martin G, Bhuyan M, Troles J, D’Amico C, Stoian R, Le Coarer E (2017) Near infrared spectrointerferometer using femtosecond laser written GLS embedded waveguides and nano-scatterers. Opt Express 25:8386. https://doi.org/10.1364/OE.25.008386

Martin S, Serabyn E, Liewer K, Loya F, Mennesson B, Hanot C, Mawet D (2008) The development and applications of a ground-based fiber nulling coronagraph. In: Optical and infrared interferometry. Proceedings of SPIE, vol 7013. p 70131Y. https://doi.org/10.1117/12.789484

Mathieu RD (1992) The Short-Period Frequency Among Low-Mass PreMain Sequence Stars. In: McAlister HA, Hartkopf WI (eds) Complementary approaches to double and multiple star research. IAU Colloquium, vol 32. pp 30-40. https://doi.org/10.1017/S1743921307009672

Mawet D, Riaud P, Absil O, Surdej J (2005) Annular groove phase mask coronagraph. AJ 633:1191-1200

Mawet D, Serabyn E, Liewer K, Hanot C, McEldowney S, Shemo D, O’Brien N (2009) Optical vectorial vortex coronagraphs using liquid crystal polymers: theory, manufacturing and laboratory demonstration. Opt Express 17:1902-1918. https://doi.org/10.1364/OE.17.001902

Mawet D, Serabyn E, Liewer K, Burruss R, Hickey J, Shemo D (2010) The vector vortex coronagraph: laboratory results and first light at palomar observatory. ApJ 709:53-57. https://doi.org/10.1088/ 0004-637X/709/1/53

Mawet D, Pueyo L, Lawson P, Mugnier L, Traub W, Boccaletti A, Trauger JT, Gladysz S, Serabyn E, Milli J, Belikov R, Kasper M, Baudoz P, Macintosh B, Marois C, Oppenheimer B, Barrett H, Beuzit JL, Devaney N, Girard J, Guyon O, Krist J, Mennesson B, Mouillet D, Murakami N, Poyneer L, Savransky D, Vérinaud C, Wallace JK (2012) Review of small-angle coronagraphic techniques in the wake of ground-based second-generation adaptive optics systems. In: Space Telescopes and Instrumentation 2012: Optical, Infrared, and Millimeter Wave. Proceedings of SPIE, vol 8442. p 844204. https://doi.org/10.1117/12.927245. arXiv:1207.5481 [astro-ph.IM]

Mawet D, Absil O, Delacroix C, Girard JH, Milli J, O’Neal J, Baudoz P, Boccaletti A, Bourget P, Christiaens V, Forsberg P, Gonte F, Habraken S, Hanot C, Karlsson M, Kasper M, Lizon JL, Muzic K, Olivier R, na EP, Slusarenko N, Tacconi-Garman LE, Surdej J, (2013) L'-band AGPM vector vortex coronagraph's first light on VLT/NACO. Discovery of a late-type companion at two beam widths from an F0V star. A\&A 553:L13. https://doi.org/10.1051/0004-6361/201321315

Mawet D, Pueyo L, Carlotti A, Mennesson B, Serabyn E, Wallace JK (2013) Ring-apodized vortex coronagraphs for obscured telescopes. I. Transmissive ring apodizers. ApJS 209:7. https://doi.org/ 10.1088/0067-0049/209/1/7. arXiv:1309.3328 [astro-ph.IM]

Mayor M, Queloz D (1995) A Jupiter-mass companion to a solar-type star. Nature 378:355-359. https:// doi.org/10.1038/378355a0

Mayor M, Udry S, Lovis C, Pepe F, Queloz D, Benz W, Bertaux JL, Bouchy F, Mordasini C, Segransan D (2009) The HARPS search for southern extra-solar planets. XIII. A planetary system with 3 super- 
Earths (4.2, 6.9, and 9.2 $\mathrm{M}_{\oplus}$ ). A\&A 493:639-644. https://doi.org/10.1051/0004-6361:200810451. arXiv:0806.4587

McCabe C, Ghez AM, Prato L, Duchêne G, Fisher RS, Telesco C (2006) Investigating disk evolution: a high spatial resolution mid-infrared survey of T Tauri stars. ApJ 636:932-951. https://doi.org/10. 1086/498207

McGregor PJ, Hart J, Conroy PG, Pfitzner ML, Bloxham GJ, Jones DJ, Downing MD, Dawson M, Young P, Jarnyk M et al (2003) Gemini near-infrared integral field spectrograph (NIFS). In: Iye M, Moorwood AFM (eds) Instrument Design and Performance for Optical/Infrared Ground-based Telescopes. Proceedings of SPIE, vol 4841. SPIE, p 1581. https://doi.org/10.1117/12.459448

McMahon DH (1975) Efficiency limitations imposed by thermodynamics on optical coupling in fiberoptic data links. J Opt Soc Am 65:1479

Mennesson B, Millan-Gabet R, Serabyn E, Colavita MM, Absil O, Bryden G, Wyatt M, Danchi W, Defrère D, Doré O, Hinz P, Kuchner M, Ragland S, Scott N, Stapelfeldt K, Traub W, Woillez J (2014a) Constraining the exozodiacal luminosity function of main-sequence stars: complete results from the Keck Nuller mid-infrared surveys. ApJ 797:119. https://doi.org/10.1088/0004-637X/797/2/ 119

Mennesson B, Millan-Gabet R, Serabyn E, Colavita MM, Absil O, Bryden G, Wyatt M, Danchi W, Defrère D, Doré O, Hinz P, Kuchner M, Ragland S, Scott N, Stapelfeldt K, Traub W, Woillez J (2014b) Constraining the exozodiacal luminosity function of main-sequence stars: complete results from the Keck Nuller Mid-infrared surveys. ApJ 797:119. https://doi.org/10.1088/0004-637X/797/2/ 119

Millan-Gabet R, Serabyn E, Mennesson B, Traub WA, Barry RK, Danchi WC, Kuchner M, Stark CC, Ragland S, Hrynevych M, Woillez J, Stapelfeldt K, Bryden G, Colavita MM, Booth AJ (2011) Exozodiacal dust levels for nearby main-sequence stars: a survey with the Keck Interferometer Nuller. ApJ 734:67. https://doi.org/10.1088/0004-637X/734/1/67. arXiv:1104.1382 [astro-ph.SR]

Miller DAB (2013) Self-aligning universal beam coupler. Opt Express 21(5):6360-6370. https://doi.org/ 10.1364/OE.21.006360

Millour F, Meilland A, Chesneau O, Stee P, Kanaan S, Petrov R, Mourard D, Kraus S (2011a) Imaging the spinning gas and dust in the disc around the supergiant A[e] star HD 62623. A\&A 526:A107. https://doi.org/10.1051/0004-6361/201016193. arXiv:1012.2957 [astro-ph.SR]

Millour F, Meilland A, Chesneau O, Stee P, Kanaan S, Petrov R, Mourard D, Kraus S (2011b) Imaging the spinning gas and dust in the disc around the supergiant A[e] star HD 62623. A\&A 526:A107. https://doi.org/10.1051/0004-6361/201016193. arXiv:1012.2957 [astro-ph.SR]

Minardi S, Pertsch T (2010) Interferometric beam combination with discrete optics. Opt Lett 35:3009-3011. https://doi.org/10.1364/OL.35.003009

Minardi S, Dreisow F, Gräfe M, Nolte S, Pertsch T (2012) Three-dimensional photonic component for multichannel coherence measurements. Opt Lett 37:3030-3032. https://doi.org/10.1364/OL.37. 003030

Minardi S, Lacour S, Berger JP, Labadie L, Thomson RR, Haniff C, Ireland M (2016) Beam combination schemes and technologies for the planet formation imager. In: Optical and Infrared Interferometry and Imaging V. Proceedigs of SPIE, vol 9907. p 99071N. https://doi.org/10.1117/12.2232656. arXiv:1608.00586 [astro-ph.IM]

Molaro P, Esposito M, Monai S, Lo Curto G, González Hernández JI, Hänsch TW, Holzwarth R, Manescau A, Pasquini L, Probst RA, Rebolo R, Steinmetz T, Udem T, Wilken T (2013) A frequency comb calibrated solar atlas. A\&A 560:A61. https://doi.org/10.1051/0004-6361/201322324. arXiv: 1310.5087 [astro-ph.SR]

Monnier JD, Millan-Gabet R (2002) On the interferometric sizes of young stellar objects. ApJ 579:694-698. https://doi.org/10.1086/342917. arXiv:astro-ph/0207292

Monnier JD, Berger JP, Millan-Gabet R, ten Brummelaar TA (2004) The Michigan Infrared Combiner (MIRC): IR imaging with the CHARA Array. In: Traub WA (ed) New frontiers in stellar interferometry. Proceedings of SPIE, vol 5491. p 1370. https://doi.org/10.1117/12.550804

Monnier JD, Zhao M, Pedretti E, Thureau N, Ireland M, Muirhead P, Berger JP, Millan-Gabet R, Van Belle G, ten Brummelaar T, McAlister H, Ridgway S, Turner N, Sturmann L, Sturmann J, Berger D (2007) Imaging the Surface of Altair. Science 317(5836):342. https://doi.org/10.1126/science. 1143205

Murakami N, Hamaguchi S, Sakamoto M, Fukumoto R, Ise A, Oka K, Baba N, Tamura M (2013) Design and laboratory demonstration of an achromatic vector vortex coronagraph. Opt Express 21:7400. https://doi.org/10.1364/OE.21.007400 
Murray GJ, Allington-Smith JR (2009) Strategies for spectroscopy on extremely large telescopes-ii. diverse-field spectroscopy. MNRAS 399(1):209-218

Norris B, Cvetojevic N, Gross S, Jovanovic N, Stewart PN, Charles N, Lawrence JS, Withford MJ, Tuthill P (2014) High-performance 3d waveguide architecture for astronomical pupil-remapping interferometry. Opt Express 22(15):18335-18353. https://doi.org/10.1364/OE.22.018335

Norris BRM, Cvetojevic N, Lagadec T, Jovanovic N, Gross S, Arriola A, Gretzinger T, Martinod MA, Guyon O, Lozi J, Withford MJ, Lawrence JS, Tuthill P (2020) First on-sky demonstration of an integrated-photonic nulling interferometer: the GLINT instrument. MNRAS 491(3):4180-4193. https://doi.org/10.1093/mnras/stz3277. arXiv:1911.09808 [astro-ph.IM]

Norris BRM, Wei J, Betters CH, Wong A, Leon-Saval SG (2020) All-fibre focal-plane wavefront sensor. In: 14th Pacific Rim Conference on Lasers and Electro-Optics (CLEO PR 2020). OSA, p PDP_1. https://doi.org/10.1364/CLEOPR.2020.PDP_1

Offer AR, Bland-Hawthorn J (1998) Rugate filters for OH-suppressed imaging at near-infrared wavelengths. MNRAS 299:176-188. https://doi.org/10.1046/j.1365-8711.1998.01760.x. arXiv: astro-ph/9707298

Ohnaka K, Weigelt G, Hofmann KH (2017) Vigorous atmospheric motion in the red supergiant star Antares. Nature 548:310-312. https://doi.org/10.1038/nature23445. arXiv:1708.06372 [astro-ph.SR]

Oppenheimer BR, Hinkley S (2009) High-Contrast Observations in Optical and Infrared Astronomy. ARA\&A 47:253-289. https://doi.org/10.1146/annurev-astro-082708-101717. arXiv:0903.4466 [astro-ph.IM]

Oshagh M, Dreizler S, Santos NC, Figueira P, Reiners A (2016) Can stellar activity make a planet seem misaligned? A\&A 593:A25. https://doi.org/10.1051/0004-6361/201628728

Padgett DL, Brandner W, Stapelfeldt KR, Strom SE, Terebey S, Koerner D (1999) Hubble Space Telescope/NICMOS Imaging of Disks and Envelopes around Very Young Stars. AJ 117:1490-1504. https://doi.org/10.1086/300781. arXiv:astro-ph/9902101

Paladini C, Baron F, Jorissen A, Le Bouquin JB, Freytag B, van Eck S, Wittkowski M, Hron J, Chiavassa A, Berger JP, Siopis C, Mayer A, Sadowski G, Kravchenko K, Shetye S, Kerschbaum F, Kluska J, Ramstedt S (2018) Large granulation cells on the surface of the giant star $\pi^{1}$ Gruis. Nature 553:310-312. https://doi.org/10.1038/nature25001

Pasquini L, Avila G, Blecha A, Cacciari C, Cayatte V, Colless M, Damiani F, de Propris R, Dekker H, di Marcantonio P, Farrell T, Gillingham P, Guinouard I, Hammer F, Kaufer A, Hill V, Marteaud M, Modigliani A, Mulas G, North P, Popovic D, Rossetti E, Royer F, Santin P, Schmutzer R, Simond G, Vola P, Waller L, Zoccali M (2002) Installation and commissioning of FLAMES, the VLT Multifibre Facility. The Messenger 110:1-9

Paufique J, Argomedo J, Arsenault R, Conzelmann R, Donaldson R, Hubin N, Jochum L, Jost A, Kiekebusch M, Kolb J, Kuntschner H, Louarn ML, Madec PY, Siebenmorgen R, Tordo S (2012) Status of the GRAAL system development: very wide-field correction with 4 laser guide-stars. In: Ellerbroek BL, Marchetti E, Véra JP (eds) Adaptive Optics Systems III. Proceedings of SPIE, vol 8447. SPIE, p 844738. https://doi.org/10.1117/12.927265

Peacock JA, Cole S, Norberg P, Baugh CM, Bland-Hawthorn J, Bridges T, Cannon RD, Colless M, Collins C, Couch W et al (2001) A measurement of the cosmological mass density from clustering in the 2dF Galaxy Redshift Survey. Nature 410(6825):169. https://doi.org/10.1038/35065528

Pedichini F, Stangalini M, Ambrosino F, Puglisi A, Pinna E, Bailey V, Carbonaro L, Centrone M, Christou J, Esposito S, Farinato J, Fiore F, Giallongo E, Hill JM, Hinz PM, Sabatini L (2017) High contrast imaging in the visible: first experimental results at the Large Binocular Telescope. AJ 154:74. https://doi.org/10.3847/1538-3881/aa7ff3. arXiv:1609.05147 [astro-ph.IM]

Pedretti E, Piacentini S, Corrielli G, Osellame R, Minardi S (2018) A six-apertures discrete beam combiners for J-band interferometry. In: Creech-Eakman MJ, Tuthill PG, Mérand A (eds) Optical and infrared interferometry and imaging VI. Proceedings of SPIE, vol 10701. SPIE, p 1070116. https://doi.org/10.1117/12.2312033

Pepe FA, Cristiani S, Rebolo Lopez R, Santos NC, Amorim A, Avila G, Benz W, Bonifacio P, Cabral A, Carvas P, Cirami R, Coelho J, Comari M, Coretti I, De Caprio V, Dekker H, Delabre B, Di Marcantonio P, D’Odorico V, Fleury M, García R, Herreros Linares JM, Hughes I, Iwert O, Lima J, Lizon JL, Lo Curto G, Lovis C, Manescau A, Martins C, Mégevand D, Moitinho A, Molaro P, Monteiro M, Monteiro M, Pasquini L, Mordasini C, Queloz D, Rasilla JL, Rebordão JM, Santana Tschudi S, Santin P, Sosnowska D, Spanò P, Tenegi F, Udry S, Vanzella E, Viel M, Zapatero Osorio MR, Zerbi F (2010) ESPRESSO: the Echelle spectrograph for rocky exoplanets and 
stable spectroscopic observations. In: McLean IS, Ramsay SK, Takami H (eds) Ground-based and Airborne Instrumentation for Astronomy III. Proceedings of SPIE, vol 7735, p 77350F. https://doi. org/10.1117/12.857122

Perger M, Scandariato G, Ribas I, Morales JC, Affer L, Azzaro M, Amado PJ, Anglada-Escudé G, Baroch D, Barrado D, Bauer FF, Béjar VJS, Caballero JA, Cortés-Contreras M, Damasso M, Dreizler S, González-Cuesta L, González Hernández JI, Guenther EW, Henning T, Herrero E, Jeffers SV, Kaminski A, Kürster M, Lafarga M, Leto G, López-González MJ, Maldonado J, Micela G, Montes D, Pinamonti M, Quirrenbach A, Rebolo R, Reiners A, Rodríguez E, Rodríguez-López C, Schmitt JHMM, Sozzetti A, Suárez Mascareño A, Toledo-Padrón B, Zanmar Sánchez R, Zapatero Osorio MR, Zechmeister M (2019) Gliese 49: activity evolution and detection of a super-Earth. A HADES and CARMENES collaboration. A\&A 624:A123. https://doi.org/10.1051/0004-6361/201935192

Perrin G, Lai O, Lena PJ, Coudé du Foresto V (2000) Fibered large interferometer on top of Mauna Kea: OHANA, the optical Hawaiian array for nanoradian astronomy. In: Léna P, Quirrenbach A (eds) Interferometry in Optical Astronomy. Proc. SPIE., vol 4006. SPIE, pp 708-714. https://doi.org/10. $1117 / 12.390272$

Perrin G, Woillez J, Lai O, Guérin J, Kotani T, Wizinowich PL, Mignant DL, Hrynevych M, Gathright J, Léna P, Chaffee F, Vergnole S, Delage L, Reynaud F, Adamson AJ, Berthod C, Brient B, Collin C, Crétenet J, Dauny F, Deléglise C, Fédou P, Goeltzenlichter T, Guyon O, Hulin R, Marlot C, Marteaud M, Melse BT, Nishikawa J, Reess JM, Ridgway ST, Rigaut F, Roth K, Tokunaga AT, Zieglera D (2006) Interferometric coupling of the Keck telescopes with single-mode fibers. Science 311:194. https://doi.org/10.1126/science.1120249

Perrin MD, Duchêne G, Kalas P, Graham JR (2006) Discovery of an optically thick, edge-on disk around the Herbig Ae star PDS 144N. ApJ 645:1272-1282. https://doi.org/10.1086/504510. arXiv:astro-ph/ 0603667

Peters MA, Close LM, Rademacher M, Stalcup T, Swartzlander GA, Ford E, Abdul-Malik RS (2008) A high-Strehl low-resolution optical imager (BESSEL): detection of a $0.7 \lambda / D$ separation binary from the ground. New Astron 13:359-369. https://doi.org/10.1016/j.newast.2007.11.008

Peters MA, Groff T, Kasdin NJ, McElwain MW, Galvin M, Carr MA, Lupton R, Gunn JE, Knapp G, Gong Q, et al. (2012) Conceptual design of the Coronagraphic High Angular Resolution Imaging Spectrograph (CHARIS) for the Subaru Telescope. In: McLean IS, Ramsay SK, Takami H (eds) Ground-based and airborne instrumentation for astronomy IV. Proceedings of SPIE, vol 8446. SPIE, p 84467U. https://doi.org/10.1117/12.926381

Petrov RG, Malbet F, Weigelt G, Antonelli P, Beckmann U, Bresson Y, Chelli A, Dugué M, Duvert G, Gennari S, Glück L, Kern P, Lagarde S, Le Coarer E, Lisi F, Millour F, Perraut K, Puget P, Rantakyrö F, Robbe-Dubois S, Roussel A, Salinari P, Tatulli E, Zins G, Accardo M, Acke B, Agabi K, Altariba E, Arezki B, Aristidi E, Baffa C, Behrend J, Blöcker T, Bonhomme S, Busoni S, Cassaing F, Clausse JM, Colin J, Connot C, Delboulbé A, Domiciano de Souza A, Driebe T, Feautrier P, Ferruzzi D, Forveille T, Fossat E, Foy R, Fraix-Burnet D, Gallardo A, Giani E, Gil C, Glentzlin A, Heiden M, Heininger M, Hernandez Utrera O, Hofmann KH, Kamm D, Kiekebusch M, Kraus S, Le Contel D, Le Contel JM, Lesourd T, Lopez B, Lopez M, Magnard Y, Marconi A, Mars G, Martinot-Lagarde G, Mathias P, Mège P, Monin JL, Mouillet D, Mourard D, Nussbaum E, Ohnaka K, Pacheco J, Perrier C, Rabbia Y, Rebattu S, Reynaud F, Richichi A, Robini A, Sacchettini M, Schertl D, Schöller M, Solscheid W, Spang A, Stee P, Stefanini P, Tallon M, Tallon-Bosc I, Tasso D, Testi L, Vakili F, von der Lühe O, Valtier JC, Vannier M, Ventura N (2007) AMBER, the near-infrared spectro-interferometric three-telescope VLTI instrument. A\&A 464:1-12. https://doi. org/10.1051/0004-6361:20066496

Phillips DF, Glenday AG, Li CH, Cramer C, Furesz G, Chang G, Benedick AJ, Chen LJ, Kärtner FX, Korzennik S, Sasselov D, Szentgyorgyi A, Walsworth RL (2012) Calibration of an astrophysical spectrograph below $1 \mathrm{~m} / \mathrm{s}$ using a laser frequency comb. Opt Express 20:13711-13726. https://doi. org/10.1364/OE.20.013711

Pojmanski G (1997) The all sky automated survey. Acta Astron 47:467-481 arXiv:astro-ph/9712146

Pontoppidan KM, Dullemond CP, van Dishoeck EF, Blake GA, Boogert ACA, Evans NJ II, KesslerSilacci JE, Lahuis F (2005) Ices in the Edge-on Disk CRBR 2422.8-3423: Spitzer Spectroscopy and Monte Carlo Radiative Transfer Modeling. ApJ 622:463-481. https://doi.org/10.1086/427688. arXiv:astro-ph/0411367

Pontoppidan KM, Blake GA, van Dishoeck EF, Smette A, Ireland MJ, Brown J (2008) Spectroastrometric imaging of molecular gas within protoplanetary disk gaps. ApJ 684:1323-1329. https://doi.org/10. 1086/590400. arXiv:0805.3314 
Primmerman CA, Murphy DV, Page DA, Zollars BG, Barclay HT (1991) Compensation of atmospheric optical distortion using a synthetic beacon. Nature 353:141-143. https://doi.org/10.1038/353141a0

Rabien, S, Angel, R, Barl, L, Beckmann, U, Busoni, L, Belli, S, Bonaglia, M, Borelli, J, Brynnel, J, Buschkamp, P, Cardwell, A, Contursi, A, Connot, C, Davies, R, Deysenroth, M, Durney, O, Eisenhauer, F, Elberich, M, Esposito, S, Frye, B, Gaessler, W, Gasho, V, Gemperlein, H, Genzel, R, Georgiev, I Y, Green, R, Hart, M, Kohlmann, C, Kulas, M, Lefebvre, M, Mazzoni, T, Noenickx, J, Orban de Xivry, G, Ott, T, Peter, D, Puglisi, A, Qin, Y, Quirrenbach, A, Raab, W, Rademacher, M, Rahmer, G, Rosensteiner, M, Rix, H W, Salinari, P, Schwab, C, Sivitilli, A, Steinmetz, M, Storm, J, Veillet, C, Weigelt, G, Ziegleder, J (2019) ARGOS at the LBT. Binocular laser guided ground-layer adaptive optics. A\&A 621:A4. https://doi.org/10.1051/0004-6361/201833716

Racine R, Walker GA, Nadeau D, Doyon R, Marois C (1999) Speckle noise and the detection of faint companions. PASP 111(759):587. https://doi.org/10.1086/316367

Rawson EG, Goodman JW, Norton RE (1980) Frequency dependence of modal noise in multimode optical fibers. J Opt Soc Am 70(8):968-976. https://doi.org/10.1364/JOSA.70.000968

Rebolo R, Osorio MRZ, Martin EL (1995) Discovery of a brown dwarf in the pleiades star cluster. Nature 377(6545):129. https://doi.org/10.1038/377129a0

Renard S, Malbet F, Benisty M, Thiébaut E, Berger JP (2010) Milli-arcsecond images of the Herbig Ae star HD 163296. A\&A 519:A26. https://doi.org/10.1051/0004-6361/201014910. arXiv:1007.2930 [astro-ph.SR]

Reynaud F, Alleman JJ, Connes P (1992) Interferometric control of fiber lengths for a coherent telescope array. Appl Opt 31:3736-3743. https://doi.org/10.1364/AO.31.003736

Rhodes WT, Goodman JW (1973) Interferometric technique for recording and restoring images degraded by unknown aberrations. J Opt Soc Am 63:647-657

Robinson B, Boroson D, Burianek D, Murphy D, Khatri F, Biswas A, Sodnik Z, Burnside J, Kansky J, M Cornwell D (2014) The NASA lunar laser communication demonstration-successful high-rate laser communications to and from the Moon. In: SpaceOps 2014 Conference, pp AIAA 2014-1685. https://doi.org/10.2514/6.2014-1685

Roddier F, Roddier C (1997) Stellar coronagraph with phase mask. PASP 109:815-820

Ródenas A, Martin G, Arzeki B, Psaila ND, Jose G, Jha A, Labadie L, Kern P, Kar AK, Thomson RR (2012) Three-dimensional mid-infrared photonic circuits in chalcogenide glass. Opt Lett 37:392-394. https://doi.org/10.1364/OL.37.000392

Roettenbacher RM, Monnier JD, Korhonen H, Aarnio AN, Baron F, Che X, Harmon RO, Kővári Z, Kraus S, Schaefer GH, Torres G, Zhao M, Ten Brummelaar TA, Sturmann J, Sturmann L (2016) No sunlike dynamo on the active star $\zeta$ Andromedae from starspot asymmetry. Nature 533:217-220. https:// doi.org/10.1038/nature17444. arXiv:1709.10107 [astro-ph.SR]

Rogstad DH (1968) A technique for measuring the visibility phase with an optical interferometer in the presence of atmospheric seeing. Appl Opt 7:585. https://doi.org/10.1364/AO.7.000585

Rohloff RR, Leinert C (1991) Properties of fiber optics for application in astronomical interferometry. Appl Opt 30:5031-5036. https://doi.org/10.1364/AO.30.005031

Rouan D, Riaud P, Boccaletti A, Clénet Y, Labeyrie A (2000) The four-quadrant phase-mask coronagraph. I. Principle. Pub Astron Soc Pac 112:1479-1486. https://doi.org/10.1086/317707

Ruane GJ, Kanburapa P, Han J, Swartzlander GA (2014) Vortex-phase filtering technique for extracting spatial information from unresolved sources. Appl Opt 53:4503. https://doi.org/10.1364/AO.53. 004503. arXiv:1401.7831 [physics.optics]

Sahlmann J, Henning T, Queloz D, Quirrenbach A, Elias NM, Launhardt R, Pepe F, Reffert S, Ségransan D, Setiawan J, Abuter R, Andolfato L, Bizenberger P, Baumeister H, Chazelas B, Delplancke F, Dérie F, Di Lieto N, Duc TP, Fleury M, Graser U, Kaminski A, Köhler R, Lévêque S, Maire C, Mégevand D, Mérand A, Michellod Y, Moresmau JM, Mohler M, Müller A, Müllhaupt P, Naranjo V, Sache L, Salvade Y, Schmid C, Schuhler N, Schulze-Hartung T, Sosnowska D, Tubbs B, van Belle GT, Wagner K, Weber L, Zago L, Zimmerman N (2013) The ESPRI project: astrometric exoplanet search with PRIMA. I. Instrument description and performance of first light observations. A\&A 551:A52. https://doi.org/10.1051/0004-6361/201220569. arXiv:1212.2227 [astro-ph.IM]

Saito N, Akagawa K, Ito M, Takazawa A, Saito YHY, Ito M, Takami H, Iye M, Wada S (2007) Sodium $\mathrm{D}_{2}$ resonance radiation single pass sum-frequency generation with actively mode-locked Nd:YAG lasers. Opt Lett 32:1965-1967. https://doi.org/10.1364/OL.32.001965

Saito Y, Hayano Y, Ito M, Minowa Y, Egner S, Oya S, Watanabe M, Hattori M, Garrel V, Akagawa K, Guyon O, Colley S, Golota T, Saito N, Takazawa A, Ito M, Takami H, Wada S, Iye M (2010) The performance of the laser guide star system for the Subaru Telescope. In: Ellerbroek BL, Hart M, 
Hubin N, Wizinowich PL (eds) Adaptive optics systems II. Proceedings of SPIE, vol 7736. SPIE, p 53. https://doi.org/10.1117/12.856484

Sana H, Le Bouquin JB, Lacour S, Berger JP, Duvert G, Gauchet L, Norris B, Olofsson J, Pickel D, Zins G, Absil O, de Koter A, Kratter K, Schnurr O, Zinnecker H (2014) Southern massive stars at high angular resolution: observational campaign and companion detection. ApJS 215:15. https://doi.org/ 10.1088/0067-0049/215/1/15. arXiv:1409.6304 [astro-ph.SR]

Saviauk A, Minardi S, Dreisow F, Nolte S, Pertsch T (2013) 3D-integrated optics component for astronomical spectro-interferometry. Appl Opt 52:4556. https://doi.org/10.1364/AO.52.004556

Schödel R, Ott T, Genzel R, Hofmann R, Lehnert M, Eckart A, Mouawad N, Alexander T, Reid MJ, Lenzen R, Hartung M, Lacombe F, Rouan D, Gendron E, Rousset G, Lagrange AM, Brandner W, Ageorges N, Lidman C, Moorwood AFM, Spyromilio J, Hubin N, Menten KM (2002) A star in a 15.2-year orbit around the supermassive black hole at the centre of the Milky Way. Nature 419:694-696. https://doi.org/10.1038/nature01121. arXiv:astro-ph/0210426

Schroeder DJ (1987) Astronomical optics. Academic Press, San Diego

Seemann U, Anglada-Escude G, Baade D, Bristow P, Dorn RJ, Follert R, Gojak D, Grunhut J, Hatzes AP, Heiter U, Ives DJ, Jeep P, Jung Y, Käufl HU, Kerber F, Klein B, Lizon JL, Lockhart M, Löwinger T, Marquart T, Oliva E, Paufique J, Piskunov N, Pozna E, Reiners A, Smette A, Smoker J, Stempels E, Valenti E (2014) Wavelength calibration from $1-5 \mu \mathrm{m}$ for the CRIRES+ high-resolution spectrograph at the VLT. In: Ramsay SK, McLean IS, Takami H (eds) Ground-based and Airborne Instrumentation for Astronomy V. Proceedings of SPIE, vol 9147. p 91475G. https://doi.org/10. $1117 / 12.2056668$

Serabyn E, Wallace K, Troy M, Mennesson B, Haguenauer P, Gappinger R, Burruss R (2007) Extreme adaptive optics imaging with a clear and well-corrected off-axis telescope subaperture. ApJ 658:1386-1391. https://doi.org/10.1086/511949

Serabyn E, Mawet D, Burruss R (2010) An image of an exoplanet separated by two diffraction beamwidths from a star. Nature 464:1018-1020. https://doi.org/10.1038/nature09007

Serabyn E, Mennesson B, Colavita MM, Koresko C, Kuchner MJ (2012) The Keck Interferometer Nuller. ApJ 748:55. https://doi.org/10.1088/0004-637X/748/1/55

Serabyn E, Huby E, Matthews K, Mawet D, Absil O, Femenia B, Wizinowich P, Karlsson M, Bottom M, Campbell R, Carlomagno B, Defrère D, Delacroix C, Forsberg P, Gomez Gonzalez C, Habraken S, Jolivet A, Liewer K, Lilley S, Piron P, Reggiani M, Surdej J, Tran H, Vargas Catalán E, Wertz O (2017) The W. M. Keck Observatory Infrared Vortex Coronagraph and a First Image of HIP 79124 B. AJ 153:43. https://doi.org/10.3847/1538-3881/153/1/43. arXiv:1612.03093 [astro-ph.SR]

Shaklan S, Roddier F (1987) Single-mode fiber optics in long-baseline interferometer. Appl Opt 26:2159-2163. https://doi.org/10.1364/AO.26.002159

Shaklan S, Roddier F (1988) Coupling starlight into single-mode optical fiber optics. Appl Opt 27:2334-2338. https://doi.org/10.1364/AO.27.002334

Shaklan S, Reynaud F, Froehly C (1992) Multimode fiber-optic broad spectral band interferometer. Appl Opt 31:749-756. https://doi.org/10.1364/AO.31.000749

Sharp R, Saunders W, Smith G, Churilov V, Correll D, Dawson J, Farrel T, Frost G, Haynes R, Heald R, et al. (2006) Performance of AAOmega: the AAT multi-purpose fiber-fed spectrograph. In: McLean IS, Iye M (eds) Ground-based and Airborne Instrumentation for Astronomy. Proceedings of SPIE, vol 6269. SPIE, p 62690G. https://doi.org/10.1117/12.671022

Sharples RM, Bender R, Lehnert MD, Ramsay Howat SK, Bremer MN, Davies RL, Genzel R, Hofmann R, Ivison RJ, Saglia R, Thatte NA (2004) KMOS: an infrared multiple-object integral field spectrograph for the ESO VLT. In: Moorwood AFM, Iye M (eds) Ground-based Instrumentation for Astronomy. Proceedings of SPIE, vol 5492. SPIE, p 1179. https://doi.org/10.1117/12.550495

Smee SA, Gunn JE, Uomoto A, Roe N, Schlegel D, Rockosi CM, Carr MA, Leger F, Dawson KS, Olmstead MD et al (2013) The multi-object, fiber-fed spectrographs for the Sloan Digital Sky Survey and the baryon oscillation spectroscopic survey. AJ 146(2):32. https://doi.org/10.1088/0004$6256 / 146 / 2 / 32$

Smith BA, Terrile RJ (1984) A circumstellar disk around $\beta$ Pictoris. Science 226:1421-1424. https://doi. org/10.1126/science.226.4681.1421

Smith D, Zuber M, Sun X, Neumann G, Cavanaugh J, McGarry J, Zagwodzki T (2006) Two-way laser link over interplanetary distance. Science 311:53. https://doi.org/10.1126/science.1120091

Snellen I, de Kok R, Birkby JL, Brandl B, Brogi M, Keller C, Kenworthy M, Schwarz H, Stuik R (2015) Combining high-dispersion spectroscopy with high contrast imaging: probing rocky planets around 
our nearest neighbors. A\&A 576:A59. https://doi.org/10.1051/0004-6361/201425018. arXiv:1503. 01136 [astro-ph.EP]

Snyder AW, Love JD (1983) Optical waveguide theory. Chapman and Hall, London. https://doi.org/10. 1007/978-1-4613-2813-1

Sodnik Z, Smit H, Sans M, Giggenbach D, Becker P, Mata Calvo R, Fuchs C, Zayer I, Lanucara M, Schulz KJ, Widmer J, Arnold F, Alonso A, Montilla I (2014) Results from a lunar laser communication experiment between NASA's LADEE satellite and ESA's optical ground station. Proceedings of international conference on space optical systems and applications (ICSOS) 2014:S2-1

Spaleniak I, Jovanovic N, Gross S, Ireland MJ, Lawrence JS, Withford MJ (2013) Integrated photonic building blocks for next-generation astronomical instrumentation II: the multimode to single mode transition. Opt Express 21(22):27197-27208. https://doi.org/10.1364/OE.21.027197

Spaleniak I, Gross S, Jovanovic N, Williams RJ, Lawrence JS, Ireland MJ, Withford MJ (2014) Multiband processing of multimode light: combining $3 \mathrm{~d}$ photonic lanterns with waveguide Bragg gratings. Laser Photon Rev 8:L1-L5. https://doi.org/10.1002/lpor.201300129

Steinmetz T, Wilken T, Araujo-Hauck C, Holzwarth R, Hänsch TW, Pasquini L, Manescau A, D'Odorico S, Murphy MT, Kentischer T et al (2008) Laser frequency combs for astronomical observations. Science 321(5894):1335-1337. https://doi.org/10.1126/science.1161030

Stoll A, Zhang Z, Haynes R, Roth M (2017) High-resolution arrayed-waveguide-gratings in astronomy: design and fabrication challenges. Photonics 4(2):30. https://doi.org/10.3390/photonics4020030

Ströbele S, Penna PL, Arsenault R, Conzelmann R, Delabre B, Duchateau M, Dorn R, Fedrigo E, Hubin N, Quentin J, Jolley P, Kiekebusch M, Kirchbauer J, Klein B, Kolb J, Kuntschner H, Louarn ML, Lizon J, Madec PY, Pettazzi L, Soenke C, Tordo S, Vernet J, Muradore R (2012) GALACSI system design and analysis. In: Ellerbroek BL, Marchetti E, Véra JP (eds) Adaptive Optics Systems III. Proceedings of SPIE, vol 8447. SPIE, p 844737. https://doi.org/10.1117/12.926110

Stürmer J, Stahl O, Schwab C, Seifert W, Quirrenbach A, Amado PJ, Ribas I, Reiners A, Caballero JA (2014) CARMENES in SPIE 2014. Building a fibre link for CARMENES. In: Navarro R, Cunningham CR, Barto AA (eds) Advances in Optical and Mechanical Technologies for Telescopes and Instrumentation. Proceedings of SPIE, vol 9151. SPIE, p 915152. https://doi.org/10.1117/12. 2056541

Su T, Scott RP, Ogden C, Thurman ST, Kendrick RL, Duncan A, Yu R, Yoo SJB (2017) Experimental demonstration of interferometric imaging using photonic integrated circuits. Opt Express 25:12653. https://doi.org/10.1364/OE.25.012653

Suh MG, Yi X, Lai YH, Leifer S, Grudinin IS, Vasisht G, Martin EC, Fitzgerald MP, Doppmann G, Wang J, Mawet D, Papp SB, Diddams SA, Beichman C, Vahala K (2019) Searching for exoplanets using a microresonator astrocomb. Nat Photon 13:25-30. https://doi.org/10.1038/s41566-018-0312-3

Swain M, Vasisht G, Akeson R, Monnier J, Millan-Gabet R, Serabyn E, Creech-Eakman M, van Belle G, Beletic J, Beichman C, Boden A, Booth A, Colavita M, Gathright J, Hrynevych M, Koresko C, Le Mignant D, Ligon R, Mennesson B, Neyman C, Sargent A, Shao M, Thompson R, Unwin S, Wizinowich P (2003) Interferometer observations of subparsec-scale infrared emission in the nucleus of NGC 4151. ApJL 596:L163-L166. https://doi.org/10.1086/379235. arXiv:astro-ph/ 0308513

Swartzlander JGA (2005) Broadband nulling of a vortex phase mask. Opt Lett 30:2876-2878. https://doi. org/10.1364/OL.30.002876

Swartzlander JGA (2006) Achromatic optical vortex lens. Opt Lett 31:2042-2044. https://doi.org/10. 1364/OL.31.002042

Taha AS, Labadie L, Pantin E, Matter A, Alvarez C, Esquej P, Grellmann R, Rebolo R, Telesco C, Wolf S (2018) The spatial extent of polycyclic aromatic hydrocarbons emission in the Herbig star HD 179218. A\&A 612:A15. https://doi.org/10.1051/0004-6361/201732008. arXiv:1711.05202 [astroph.SR]

Takami M, Bailey J, Gledhill TM, Chrysostomou A, Hough JH (2001) Circumstellar structure of RU Lupi down to au scales. MNRAS 323:177-187. https://doi.org/10.1046/j.1365-8711.2001.04172.x. arXiv: astro-ph/0103311

Tatulli E, Duvert G (2007) AMBER data reduction. New Astron Rev 51(8):682-696. https://doi.org/10. 1016/j.newar.2007.06.010

Taylor L, Fen Y, Calia DB (2010) $50 \mathrm{~W}$ visible laser source at $589 \mathrm{~nm}$ obtained via frequency doubling of three coherently combined narrow-band Raman fiber amplifiers. Opt Express 18:8540-8555 
ten Brummelaar TA, Gies DG, McAlister HA, Ridgway ST, Sturmann J, Sturmann L, Schaefer GH, Turner NH, Farrington CD, Scott NJ, Monnier JD, Ireland MJ (2016) An update on the CHARA array. In: Malbet F, Creech-Eakman MJ, Tuthill PG (eds) Optical and infrared interferometry and imaging V. Proceedings of SPIE, vol 9907, p 990703. https://doi.org/10.1117/12.2232125

Tepper J, Labadie L, Gross S, Arriola A, Minardi S, Diener R, Withford MJ (2017) Ultrafast laser inscription in ZBLAN integrated optics chips for mid-IR beam combination in astronomical interferometry. Opt Express 25(17):20642-20653. https://doi.org/10.1364/OE.25.020642

Tepper J, Labadie L, Diener R, Minardi S, Pott J-U, Thomson R, Nolte S (2017) Integrated optics prototype beam combiner for long baseline interferometry in the L and M bands. A\&A 602:A66. https://doi.org/10.1051/0004-6361/201630138

Theia Collaboration, Boehm C, Krone-Martins A, Amorim A, Anglada-Escude G, Brandeker A, Courbin F, Ensslin T, Falcao A, Freese K, Holl B, Labadie L, Leger A, Malbet F, Mamon G, McArthur B, Mora A, Shao M, Sozzetti A, Spolyar D, Villaver E, Albertus C, Bertone S, Bouy H, BoylanKolchin M, Brown A, Brown W, Cardoso V, Chemin L, Claudi R, Correia ACM, Crosta M, Crouzier A, Cyr-Racine FY, Damasso M, da Silva A, Davies M, Das P, Dayal P, de Val-Borro M, Diaferio A, Erickcek A, Fairbairn M, Fortin M, Fridlund M, Garcia P, Gnedin O, Goobar A, Gordo P, Goullioud R, Hambly N, Hara N, Hobbs D, Hog E, Holland A, Ibata R, Jordi C, Klioner S, Kopeikin S, Lacroix T, Laskar J, Le Poncin-Lafitte C, Luri X, Majumdar S, Makarov V, Massey R, Mennesson B, Michalik D, Moitinho de Almeida A, Mourao A, Moustakas L, Murray N, Muterspaugh M, Oertel M, Ostorero L, Perez-Garcia A, Platais I, de Mora JPi, Quirrenbach A, Randall L, Read J, Regos E, Rory B, Rybicki K, Scott P, Schneider J, Scholtz J, Siebert A, Tereno I, Tomsick J, Traub W, Valluri M, Walker M, Walton N, Watkins L, White G, Evans DW, Wyrzykowski L, Wyse R (2017) Theia: faint objects in motion or the new astrometry frontier. arXiv:1707.01348 [astro-ph.IM]

Thomson RR, Harris RJ, Birks TA, Brown G, Allington-Smith J, Bland-Hawthorn J (2012) Ultrafast laser inscription of a 121-waveguide fan-out for astrophotonics. Opt Lett 37(12):2331-2333

Tinney CG, Ryder SD, Ellis SC, Churilov V, Dawson J, Smith G, Waller L, Whittard J, Haynes R, Lankshear A, et al. (2004) IRIS2: a working infrared multi-object spectrograph and camera. In: Moorwood AFM, Iye M (eds) Ground-based instrumentation for astronomy. Proceedings of SPIE, vol 5492. https://doi.org/10.1117/12.550980

Traub WA, Oppenheimer BR (2010) Direct imaging of exoplanets. University of Arizona Press, Tucson Trinh CQ, Ellis SC, Bland-Hawthorn J, Lawrence JS, Horton AJ, Leon-Saval SG, Shortridge K, Bryant J, Case S, Colless M et al (2013) GNOSIS: the first instrument to use fiber Bragg gratings for $\mathrm{OH}$ suppression. AJ 145(2):51

Tuthill P, Jovanovic N, Lacour S, Lehmann A, Ams M, Marshall G, Lawrence J, Withford M, Robertson G, Ireland M, Pope B, Stewart P (2010) Photonic technologies for a pupil remapping interferometer. In: Danchi WC, Delplancke F, Rajagopal JK (eds) Optical and Infrared Interferometry II. Proceedings of SPIE, vol 7734. SPIE, p 77341P. https://doi.org/10.1117/12.856770. arXiv:1006. 2587 [astro-ph.IM]

Udalski A, Szymanski M, Kaluzny J, Kubiak M, Mateo M, Krzeminski W (1994) The optical gravitational lensing experiment: the discovery of three further microlensing events in the direction of the galactic bulge. ApJ 426:69-72

Udem T, Holzwarth R, Hänsch TW (2002) Optical frequency metrology. Nature 416:233-237. https://doi. org/10.1038/416233a

Unwin SC, Shao M, Tanner AM, Allen RJ, Beichman CA, Boboltz D, Catanzarite JH, Chaboyer BC, Ciardi DR, Edberg SJ, Fey AL, Fischer DA, Gelino CR, Gould AP, Grillmair C, Henry TJ, Johnston KV, Johnston KJ, Jones DL, Kulkarni SR, Law NM, Majewski SR, Makarov VV, Marcy GW, Meier DL, Olling RP, Pan X, Patterson RJ, Pitesky JE, Quirrenbach A, Shaklan SB, Shaya EJ, Strigari LE, Tomsick JA, Wehrle AE, Worthey G (2008) Taking the measure of the universe: precision astrometry with SIM PlanetQuest. PASP 120:38. https://doi.org/10.1086/525059. arXiv:0708.3953

van Boekel R, Min M, Leinert C, Waters LBFM, Richichi A, Chesneau O, Dominik C, Jaffe W, Dutrey A, Graser U, Henning T, de Jong J, Köhler R, de Koter A, Lopez B, Malbet F, Morel S, Paresce F, Perrin G, Preibisch T, Przygodda F, Schöller M, Wittkowski M (2004) The building blocks of planets within the 'terrestrial' region of protoplanetary disks. Nature 432:479-482. https://doi.org/ 10.1038/nature03088

van den Ancker ME, Wesselius PR, Tielens AGGM, van Dishoeck EF, Spinoglio L (1999) ISO spectroscopy of shocked gas in the vicinity of T Tauri. A\&A 348:877-887 
van Dishoeck EF (2004) ISO spectroscopy of gas and dust: from molecular clouds to protoplanetary disks. ARA\&A 42:119-167. https://doi.org/10.1146/annurev.astro.42.053102.134010. arXiv:astro-ph/0403061

Van Kerckhoven C, Tielens AGGM, Waelkens C (2002) Nanodiamonds around HD 97048 and Elias 1. A\&A 384:568-584. https://doi.org/10.1051/0004-6361:20011814

Vanderriest C (1980) A fiber-optics dissector for spectroscopy of nebulosities around quasars and similar objects. PASP 92:858-862. https://doi.org/10.1086/130764

Vargas Catalán E, Huby E, Forsberg P, Jolivet A, Baudoz P, Carlomagno B, Delacroix C, Habraken S, Mawet D, Surdej J, Absil O, Karlsson M (2016) Optimizing the subwavelength grating of L-band annular groove phase masks for high coronagraphic performance. A\&A 595:A127. https://doi.org/ 10.1051/0004-6361/201628739. arXiv:1610.05065 [astro-ph.IM]

Vergnole S, Kotani T, Perrin G, Delage L, Reynaud F (2005) Calibration of silica fibers for the optical hawaiian array for nanoradian astronomy ('OHANA): temperature dependence of differential chromatic dispersion. Opt Commun 251:115-123

Véron-Cetty MP, Véron P (2000) The emission line spectrum of active galactic nuclei and the unifying scheme. A\&A Rev 10:81-133. https://doi.org/10.1007/s001590000006

Vilas F, Smith BA (1987) Coronagraph for astronomical imaging and spectrophotometry. Appl Opt 26:664-668

Watson FG (1995) Multifiber waveguide spectrograph for astronomy? In: Barden SC (ed) Fiber Optics in Astronomical Applications. Proc. SPIE., vol 2476. SPIE, p 68. https://doi.org/10.1117/12.211840

Watson FG (1997) Waveguide spectrographs for astronomy? In: Ardeberg AL (ed) Optical Telescopes of Today and Tomorrow. Proceedings of SPIE, vol 2871. SPIE, p 1373. https://doi.org/10.1117/12.269030

Weber V, Barillot M, Haguenauer P, Kern PY, Schanen-Duport I, Labeye PR, Pujol L, Sodnik Z (2004) Nulling interferometer based on an integrated optics combiner. In: Traub WA (ed) New frontiers in stellar interferometry. Proceedings of SPIE, vol 5491. SPIE, p 842. https://doi.org/10.1117/12.550581

Welford WT, Winston R (1982) Nonconventional optical systems and brightness theroem. Appl Opt 21:1531-1533. https://doi.org/10.1364/AO.21.001531

Wildi F, Pepe F, Chazelas B, Lo Curto G, Lovis C (2010) A Fabry-Perot calibrator of the HARPS radial velocity spectrograph: performance report. In: McLean IS, Ramsay SK, Takami H (eds) Groundbased and Airborne Instrumentation for Astronomy III. Proceedings of SPIE, vol 7735. SPIE, p 77354X. https://doi.org/10.1117/12.857951

Wildi F, Pepe F, Chazelas B, Lo Curto G, Lovis C (2011) The performance of the new Fabry-Perot calibration system of the radial velocity spectrograph HARPS. In: Shaklan S (ed) Techniques and Instrumentation for Detection of Exoplanets V. Proceedings of SPIE, vol 8151. SPIE, p 81511F. https://doi.org/10.1117/12.901550

Wilken T, Curto GL, Probst RA, Steinmetz T, Manescau A, Pasquini L, González Hernández JI, Rebolo R, Hänsch TW, Udem T, Holzwarth R (2012) A spectrograph for exoplanet observations calibrated at the centimetre-per-second level. Nature 485:611-614. https://doi.org/10.1038/nature11092

Woillez J, Lai O, Perrin G, Reynaud F, Baril M, Dong Y, Fédou P (2017) AGILIS: agile guided interferometer for longbaseline imaging synthesis. Demonstration and concepts of reconfigurable optical imaging interferometers. A\&A 602:A116. https://doi.org/10.1051/0004-6361/201730500. arXiv:1703.03919 [astro-ph.IM]

Yerolatsitis S, Harrington K, Birks TA (2017) All-fibre pseudo-slit reformatters. Opt Express 25(16): 18713-18721

Yi X, Vahala K, Li J, Diddams S, Ycas G, Plavchan P, Leifer S, Sandhu J, Vasisht G, Chen P, Gao P, Gagne J, Furlan E, Bottom M, Martin EC, Fitzgerald MP, Doppmann G, Beichman C (2016) Demonstration of a near-IR line-referenced electro-optical laser frequency comb for precision radial velocity measurements in astronomy. Nat Commun 7:10436. https://doi.org/10.1038/ncomms10436. arXiv:1501.02509 [astro-ph.IM]

York DG, Adelman J, Anderson JE Jr, Anderson SF, Annis J, Bahcall NA, Bakken JA, Barkhouser R, Bastian S, Berman E et al (2000) The sloan digital sky survey: technical summary. AJ 120(3):1579

Zajnulina M, Boggio JMC, Böhm M, Rieznik AA, Fremberg T, Haynes R, Roth MM (2015) Generation of optical frequency combs via four-wave mixing processes for low- and medium-resolution astronomy. Appl Phys B 120:171-184. https://doi.org/10.1007/s00340-015-6121-1. arXiv:1704. 04915 [physics.optics]

Publisher's Note Springer Nature remains neutral with regard to jurisdictional claims in published maps and institutional affiliations. 